\title{
Deprotonative Functionalization of the Difluoromethyl Group
}

Laura Santos $^{\mathrm{I}}$, Armen Panossian ${ }^{\mathrm{I}}$, Morgan Donnard ${ }^{\mathrm{I}}$, Jean-Pierre Vors ${ }^{\mathrm{II}}$, Sergii Pazenok ${ }^{\mathrm{III}}$, David Bernier ${ }^{\mathrm{II}}$ and Frédéric R. Leroux ${ }^{*, \mathrm{I}}$

IUniversity of Strasbourg, Université de Haute-Alsace,

CNRS, UMR 7042-LIMA, ECPM,

25 Rue Becquerel, Strasbourg 67087, France

"Bayer S.A.S.,

14 Impasse Pierre Baizet,

BP99163, 69263 Lyon Cedex 09, France

IIIBayer CropScience AG,

Alfred-Nobel-Strasse 50,

40789 Monheim, Germany

\section{Supporting information}




\section{Table of contents}

$\begin{array}{ll}\text { General information } & 3\end{array}$

Optimization of the in situ quench $\quad 5$

Optimization of the sequential quench 6

Optimization of the desilylative post-functionalization $\quad 7$

$\begin{array}{ll}\text { Optimization of one-pot synthesis } & 7\end{array}$

$\begin{array}{ll}\text { Typical procedures } & 8\end{array}$

Trapping of difluoro(3-pyridyl)methyllithium with electrophiles 9

3-(Difluoro(trimethylsilyl)methyl)pyridine (2a) 9

3-((Dimethyl(phenyl)silyl)difluoromethyl)pyridine (2b) 12

3-((tert-Butyldimethylsilyl)difluoromethyl)pyridine (2c) 15

3-(Difluoromethyl-d)pyridine (2d) 18

3-(1,1-Difluoroethyl)pyridine (2e) 18

3-(1,1-Difluoropentyl)pyridine (2f) 21

3-(1,1-Difluoro-2-(trimethylsilyl)ethyl)pyridine (2g) 23

Ethyl 2,2-difluoro-2-(pyridin-3-yl)acetate (2h) 25

3-(Difluoro(phenylthio)methyl)pyridine (2i) 28

3-(Difluoro(tributylstannyl)methyl)pyridine (2j) 30

3-(difluoro(phenylselanyl)methyl)pyridine (2k) 32

2,2-Difluoro-1-phenyl-2-(pyridin-3-yl)ethan-1-ol (2l) 35

1-(4-chlorophenyl)-2,2-difluoro-2-(pyridin-3-yl)ethan-1-ol (2m) 37

1-(2,4-dichlorophenyl)-2,2-difluoro-2-(pyridin-3-yl)ethan-1-ol (2n) 40

N-(2,2-difluoro-1-phenyl-2-(pyridin-3-yl)ethyl)-4-methylbenzenesulfonamide (2o) 42

2-(2,2-Difluoro-1-phenyl-2-(pyridin-3-yl)ethyl)malononitrile (2p) 45

3-(bromodifluoromethyl)pyridine (2q) 47

3-(difluoro(p-tolylsulfinyl)methyl)pyridine (2r) 49

1-(2,4-dichlorophenyl)-2,2-difluoro-2-(pyridin-3-yl)ethan-1-one (3) 52

(E)-1-(2,4-dichlorophenyl)-2,2-difluoro-2-(pyridin-3-yl)ethan-1-one O-methyl oxime (4) 55

X-Ray crystallographic structure of 2l $\quad 57$

$\begin{array}{ll}\text { References } & 65\end{array}$ 


\section{General information}

All reactions were carried out under argon atmosphere in flame-dried glassware. Liquids and solutions were transferred with syringes or cannula purged with argon prior to use. Air- and moisture- sensitive materials were stored, protected and handled under an atmosphere of argon, with appropriate glassware. Tetrahydrofuran (THF) was freshly distilled from sodium/benzophenone prior to use. Anhydrous dimethylformamide (DMF) was used from commercial source and was stored under Argon. Technical grade solvents for extraction and chromatography (cyclohexane and ethyl acetate) were used without purification. All reagents, if commercially available, were purchased from standard suppliers (Sigma Aldrich, ABCR, Fluorochem, TCl, Alfa Aesar and Apollo scientific) and used without further purification unless otherwise stated. Reactions were cooled using acetone / dry ice / liquid nitrogen baths and heated using an oil bath on a magnetic stirrer.

\section{Reagents}

3-(Difluoromethyl)pyridine, Benzaldehyde and Chlorodimethylphenylsilane were degrading upon storage and were thus freshly distilled prior to use. TMSCl was distilled and stored over $\mathrm{CaH}_{2}$ under argon. Potassium $t$-butoxide was sublimed prior to use. $n$-BuLi (1.6 M in hexane) and $t$-BuLi (1.7 M in hexane) were purchased from Sigma Aldrich and the concentration was determined following the Wittig-Harboth double titration method ((total base)-(residual base after reaction with 1,2dibromoethane)). ${ }^{1}$ Diisopropylamine (DIPA) was distilled and stored over $\mathrm{KOH}$ under argon. 2,2,6,6Tetramethylpiperidine (HTMP) was freshly distilled and stored over $4 \AA ̊$ molecular sieves previously activated overnight at $300^{\circ} \mathrm{C}$ under vacuum.

\section{Chromatography}

Analytical thin-layer chromatography (TLC) was performed using aluminum plates coated with silica $\left(0.25 \mathrm{~mm}\right.$, Merck silica-gel $\left.60-\mathrm{F}_{254}\right)$. Flash column chromatography was performed on VWR silica gel $(40-63 \mu \mathrm{m})$ using the indicated solvents. Spots were visualized by UV light irradiation and/or by staining of the TLC plate with potassium permanganate $\left(\mathrm{KMnO}_{4}(0.3 \mathrm{~g}), \mathrm{K}_{2} \mathrm{CO}_{3}(20 \mathrm{~g})\right.$ and $\mathrm{KOH}(0.3 \mathrm{~g})$ in water $(300 \mathrm{~mL}))$, followed by heating with a heat gun if necessary.

Chiral HPLC measurements were performed on a Shimadzu system with a quaternary low-pressure LC20AD pump, an automatic SIL-20A HT injector, a CTO-10 AS oven and an SPD-M20 A diode array detector (DAD). The injection volume was $1 \mu \mathrm{L}$ and the temperature of the oven was set to $35^{\circ} \mathrm{C}$.

\section{Analytical data}

${ }^{1} \mathrm{H}$ NMR (400 or $500 \mathrm{MHz}$ ), ${ }^{19} \mathrm{~F}$ NMR ( 376 or $471 \mathrm{MHz}$ ) ${ }^{29} \mathrm{Si}$ INEPT NMR (79 or $99 \mathrm{MHz}$ ) and ${ }^{13} \mathrm{C} \mathrm{NMR} \mathrm{(101}$ or $126 \mathrm{MHz}$ ) spectra were recorded on Bruker Avance III HD 400 and $500 \mathrm{MHz}$ instruments respectively. Chemical shifts are reported as $\delta$-values in parts per million (ppm) and are referred to partially deuterated chloroform $\left(\delta\left[{ }^{1} \mathrm{H}\right]=7.26 \mathrm{ppm}\right.$ and $\left.\delta\left[{ }^{13} \mathrm{C}\right]=77.16 \mathrm{ppm}\right)$. Silicon and fluorine NMR were calibrated using ${ }^{1} \mathrm{H}$ NMR as absolute reference. Fluorobenzene $\left(\delta\left[{ }^{19} \mathrm{~F}\right]=-113.15 \mathrm{ppm}\right)$ was used as internal standard to measure yields in situ. The spectra were processed with MestreNova (Mestrelab). Multiplicities were abbreviated as s (singlet), brs (broad signal), $d$ (doublet), $t$ (triplet), $q$ (quartet), $\mathrm{m}$ (multiplet), dd (doublet of doublets), ddd (doublet of doublets of doublets). Coupling constants J were given in $\mathrm{Hz}$. If not otherwise noted, the coupling constants given are either $\mathrm{H}-\mathrm{H}$ or $\mathrm{H}$ $\mathrm{F}$ coupling constants for proton signals, and $\mathrm{C}-\mathrm{F}$ and $\mathrm{Si}-\mathrm{F}$ coupling constants for carbon and silicon signals. 
Infrared spectra were recorded on a Perkin Elmer UATR Two spectrometer coupled to a diamond window ATR. The main absorption peaks are reported in $\mathrm{cm}^{-1}$.

High resolution mass spectrometry (HRMS) analysis were performed with a Bruker MicroTOF mass analyser under ESI in positive ionization mode detection (measurement accuracy $\leq 15 \mathrm{ppm}$ ) by the analytical facility at the University of Strasbourg.

The X-ray crystallographic structure analysis was performed by the radio-crystallographic facility at the Université de Strasbourg. The analysis was carried out on a Bruker PHOTON III DUO CPAD diffractometer equipped with an Oxford Cryosystem liquid $\mathrm{N}_{2}$ device, using Mo-K $\alpha$ radiation $(\lambda=0.71073 \AA)$. 


\section{Optimization of the in situ quench}<smiles></smiles><smiles>CC(C)(F)c1cccnc1</smiles>

\section{Procedure:}

A solution of 3-(difluoromethyl)pyridine (1 equiv., $0.20 \mathrm{~mL}, 1.92 \mathrm{mmol}$ ) in THF $(7 \mathrm{~mL}$ ) was cannulated onto a solution of base and TMSCl (1 equiv., $0.25 \mathrm{~mL}, 1.92 \mathrm{mmol}$ ) in THF $(7 \mathrm{~mL}$ ) under inert atmosphere and at temperature $t_{1}$. The reaction mixture was stirred for $15 \mathrm{~min}$ at $t_{1}$ and then allowed to warm slowly to $20^{\circ} \mathrm{C}$ over $30 \mathrm{~min}$. Fluorobenzene ( 0.83 equiv., $0.15 \mathrm{~mL}, 1.59 \mathrm{mmol}$ ) was used as an internal standard in ${ }^{19} \mathrm{~F}$ NMR.

\section{Order of addition for in situ quench}

\section{Reaction conditions:}

3-(Difluoromethyl)pyridine $(0.20 \mathrm{~mL}, 1.92 \mathrm{mmol}), \mathrm{LDA}(3.85 \mathrm{mmol}), \mathrm{TMSCl}(0.25 \mathrm{~mL}, 1.92 \mathrm{mmol})$ and THF (14 mL) were used with an addition at $-78{ }^{\circ} \mathrm{C}$. Reaction mixture was stirred $15 \mathrm{~min}$ at $-78{ }^{\circ} \mathrm{C}$ and then allowed to warm to room temperature for $30 \mathrm{~min}$.

The order of addition for in situ quench has an influence in the obtained yield. An excess of electrophile was required otherwise low yields were obtained, as in addition 4 and addition 1 (scheme below). The order of addition 3 with introduction of the difluoromethyl-compound at the end appeared to be the best protocol.

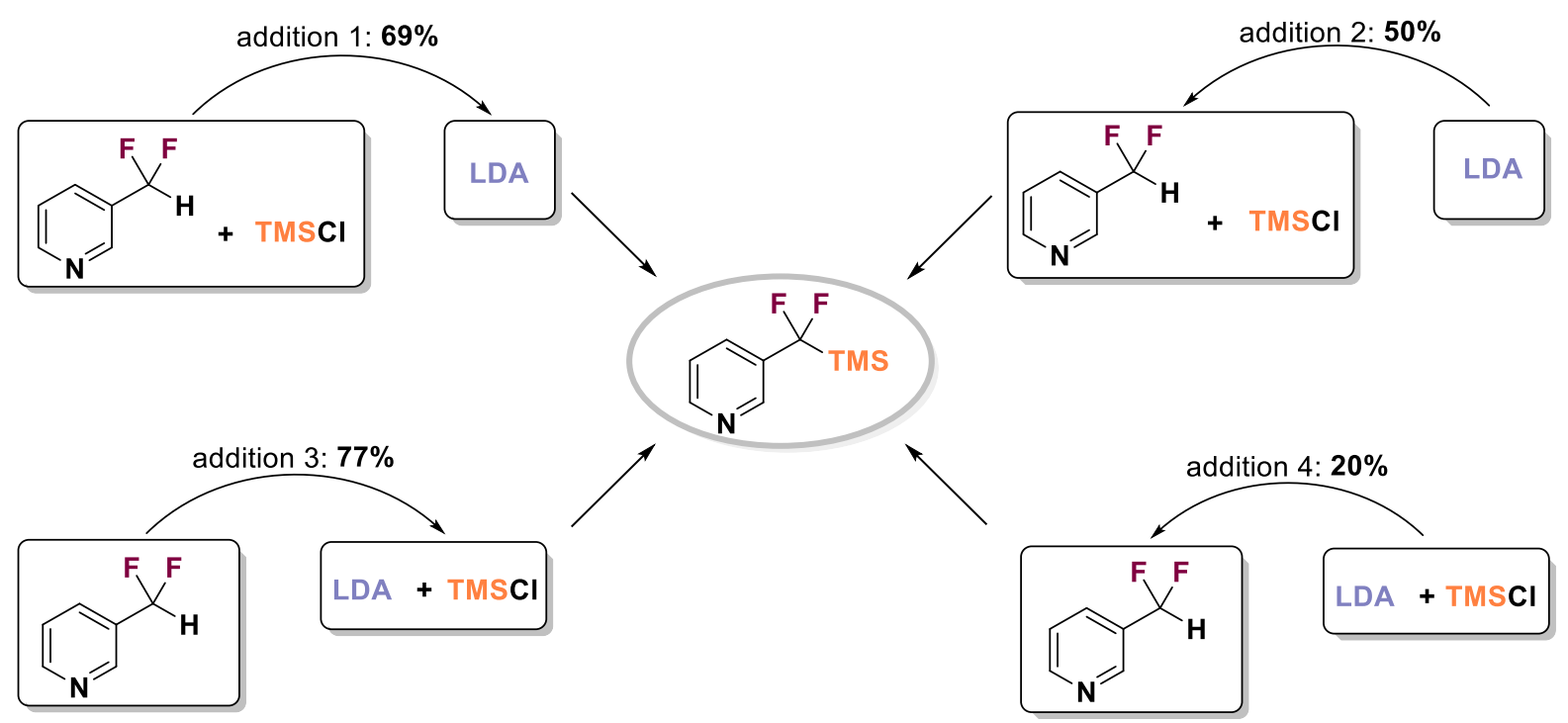




\section{Optimization of the sequential quench}

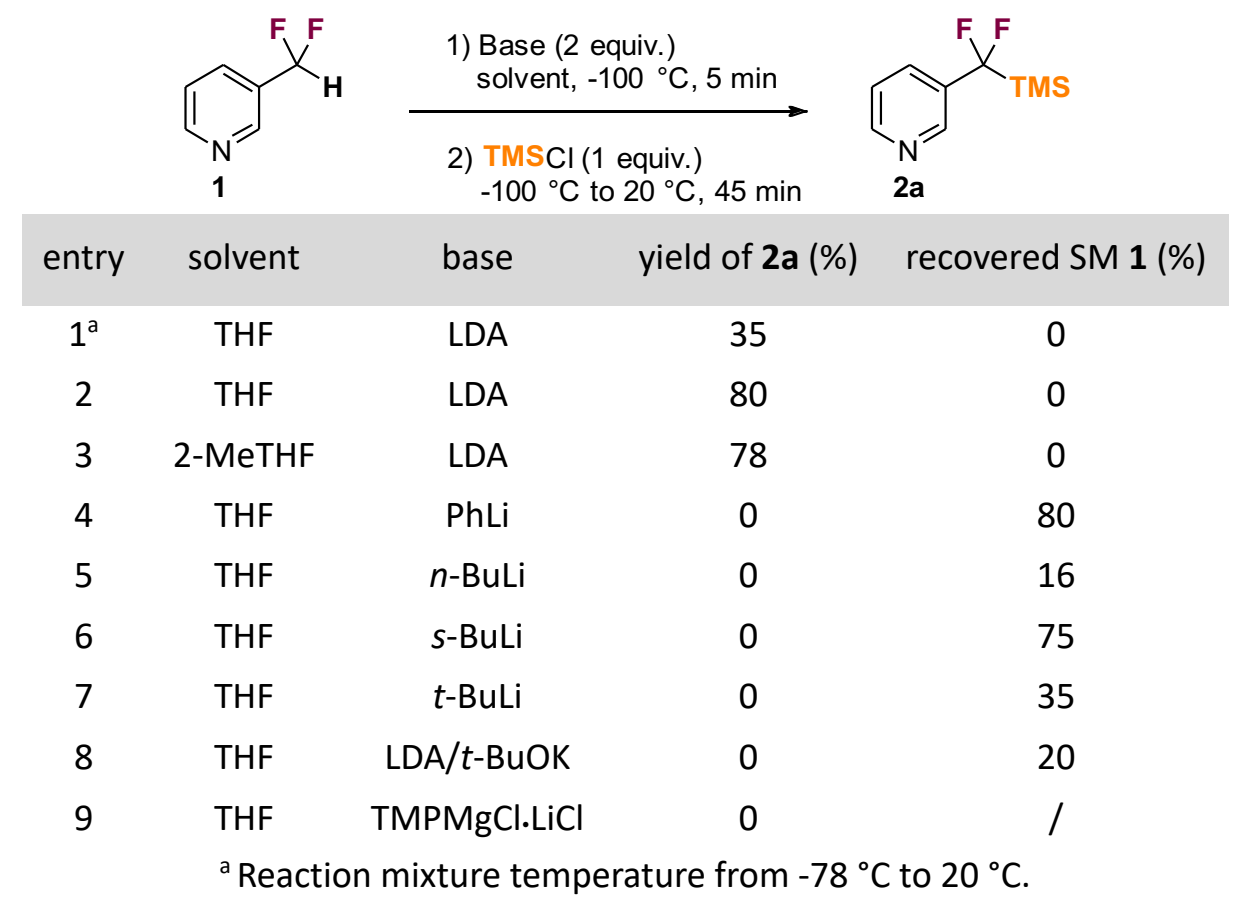

\section{Procedure:}

A solution of 3-(difluoromethyl)pyridine (1 equiv., $0.20 \mathrm{~mL}, 1.92 \mathrm{mmol}$ ) in THF $(7 \mathrm{~mL})$ was cannulated onto a solution of base ( 2 equiv.) in THF $\left(7 \mathrm{~mL}\right.$ ) under inert atmosphere and at $-100{ }^{\circ} \mathrm{C}$. The reaction mixture was stirred for $15 \mathrm{~min}$ at $-100{ }^{\circ} \mathrm{C}$ and then allowed to warm slowly to $20{ }^{\circ} \mathrm{C}$ over $30 \mathrm{~min}$. Fluorobenzene ( 0.83 equiv., $0.15 \mathrm{~mL}, 1.59 \mathrm{mmol}$ ) was used as an internal standard in ${ }^{19} \mathrm{~F} \mathrm{NMR}$.

\section{Discussion:}

When LDA was used as the base and TMSCl as electrophile at $-78{ }^{\circ} \mathrm{C}$, the desired product $2 \mathrm{a}$ was obtained with a low yield of $35 \%$ (entry 1 ). By decreasing the temperature to $-100{ }^{\circ} \mathrm{C}$, it was possible to obtain $2 \mathrm{a}$ in $80 \%$ yield (entry 2 ). Switching to the greener and process-friendly solvent MeTHF afforded a similar good yield (entry 3). Regarding other lithium bases, no formation of 2a was observed with PhLi, $n$-BuLi, $s$-BuLi or $t$-BuLi (entries 4, 5, 6 and 7, respectively). When assessing again mixed bases, such as a lithium/potassium mixed base (LDA/t-BuOK, entry 8 ) or a lithium/magnesium one (TMPMgCl.LiCl, entry 9), a complex mixture of fluorinated compounds was obtained. 


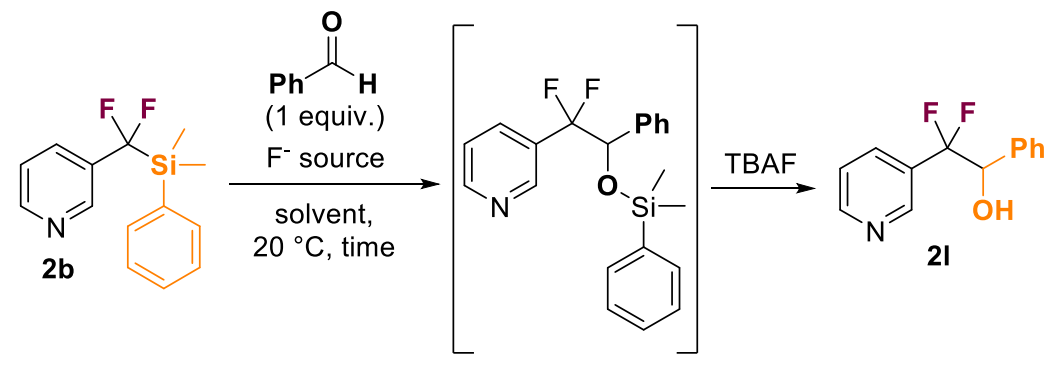

Benzaldehyde (1 equiv., $0.50 \mathrm{mmol}, 0.05 \mathrm{~mL}$ ) was added to a solution of 3((dimethyl(phenyl)silyl)difluoromethyl)pyridine (1 equiv., $0.50 \mathrm{mmol}, 132.5 \mathrm{mg}$ ) in solvent $(4 \mathrm{~mL}$ ) at 0 ${ }^{\circ} \mathrm{C}$ and under inert atmosphere. A solution of fluoride in solvent $(5 \mathrm{~mL})$ pre-cooled to $0{ }^{\circ} \mathrm{C}$ was added dropwise to the reaction mixture, which was then stirred at $20^{\circ} \mathrm{C}$ until total consumption of starting materials. ${ }^{19} \mathrm{~F}$ NMR yields were calculated with fluorobenzene as internal standard.

\section{Optimization of one-pot synthesis}

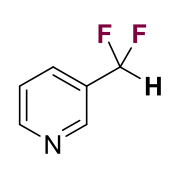

1
LDA

H

then,

activator, benzaldehyde (1 equiv.)

solvent, temperature, time

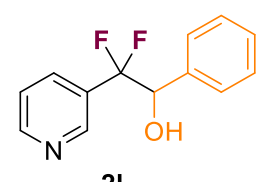

21

\begin{tabular}{cccccccc}
\hline entry & activator & solvent & time & temperature & $\begin{array}{c}\text { yield } \\
\text { of 2l }\end{array}$ & $\begin{array}{c}\text { yield } \\
\text { of } \mathbf{1}\end{array}$ & $\begin{array}{c}\text { Yield of } \\
\text { intermediate 2 b b }\end{array}$ \\
\hline 1 & TBAT (0.1 equiv.) & THF + DMF & 4 days & $20{ }^{\circ} \mathrm{C}$ & $<10 \%$ & traces & $>90 \%$ \\
2 & TBAT (0.1 equiv.) & DMF & 4 days & $20{ }^{\circ} \mathrm{C}$ & traces & traces & $>90 \%$ \\
3 & TBAT (1 equiv.) & THF + DMF & $48 \mathrm{~h}$ & $20{ }^{\circ} \mathrm{C}$ & traces & traces & $>90 \%$ \\
4 & TBAT (1 equiv.) & THF + DMF & 6 days & $20{ }^{\circ} \mathrm{C}$ & $70 \%$ & $20 \%$ & $0 \%$ \\
5 & TBAT (1 equiv.) & THF + DMF & $17 \mathrm{~h}$ & $50{ }^{\circ} \mathrm{C}$ & $29 \%$ & $20 \%$ & $50 \%$ \\
6 & $\mathrm{~K}_{2} \mathrm{CO}_{3}$ (0.1 equiv.) & $\mathrm{THF}+\mathrm{DMF}$ & $24 \mathrm{~h}$ & $20{ }^{\circ} \mathrm{C}$ & traces & traces & $>90 \%$ \\
7 & $\mathrm{~K}_{2} \mathrm{CO}_{3}$ (0.5 equiv.) & $\mathrm{THF}+\mathrm{DMF}$ & $8 \mathrm{~h}$ & $20{ }^{\circ} \mathrm{C}$ & $25 \%$ & $40 \%$ & $33 \%$ \\
8 & $\mathrm{~K}_{2} \mathrm{CO}_{3}$ (1 equiv.) & $\mathrm{DMF}$ & $20 \mathrm{~h}$ & $20{ }^{\circ} \mathrm{C}$ & $59 \%$ & $36 \%$ & $0 \%$ \\
9 & $\mathrm{~K}_{2} \mathrm{CO}_{3}$ (2 equiv.) & $\mathrm{THF}+\mathrm{DMF}$ & $38 \mathrm{~h}$ & $20{ }^{\circ} \mathrm{C}$ & $43 \%$ & $52 \%$ & $0 \%$
\end{tabular}

Procedure: 
A solution of DIPA (2 equiv., $0.27 \mathrm{~mL}, 1.92 \mathrm{mmol}$ ) in THF $(3.5 \mathrm{~mL}$ ) under inert atmosphere and cooled to $0{ }^{\circ} \mathrm{C}$ was treated with $n$-BuLi (2 equiv., $1.6 \mathrm{M}$ in hexanes, $1.27 \mathrm{~mL}, 1.92 \mathrm{mmol}$ ). After $5 \mathrm{~min}$, the solution was cooled to $-78^{\circ} \mathrm{C}$ and $\mathrm{SiMe}_{2} \mathrm{PhCl}$ (2 equiv., $0.32 \mathrm{~mL}, 1.92 \mathrm{mmol}$ ) was added. A solution of 3-(difluoromethyl)pyridine (1 equiv., $0.10 \mathrm{~mL}, 0.96 \mathrm{mmol}$ ) in THF $\left(3.5 \mathrm{~mL}\right.$ ) pre-cooled to $-78{ }^{\circ} \mathrm{C}$ was cannulated onto the first solution. The reaction mixture was stirred for $15 \mathrm{~min}$ at $-78^{\circ} \mathrm{C}$ and then slowly warmed to $20^{\circ} \mathrm{C}$ over $30 \mathrm{~min}$. Then, benzaldehyde (1 equiv., $0.1 \mathrm{~mL}, 0.96 \mathrm{mmol}$ ) and a solution of activator in DMF was added to the reaction mixture. ${ }^{19} \mathrm{~F}$ NMR yields were calculated with fluorobenzene as internal standard.

\section{Discussion:}

The one-pot reaction using TBAT in catalytic amount only afforded the formation of $\mathbf{2 l}$ in traces (entry 1 and 2). When stoichiometric amount of TBAT was used reaction was completed after 6 days (entry 3 and 4). In order to speed up the reaction mixture was stirred at $50^{\circ} \mathrm{C}$ for $17 \mathrm{~h}$ but still an incomplete conversion was obtained (entry 5). As the utilization of our best fluoride source was not working, the use of an inorganic base such as potassium carbonate was tested. A complete conversion could be obtained with $\mathrm{K}_{2} \mathrm{CO}_{3}$. When removing THF under vacuum after formation of $\mathbf{2} \mathbf{b}$ and adding DMF for the functionalization with benzaldehyde, $59 \%$ of $2 \mathrm{l}$ were obtained after $20 \mathrm{~h}$.

\section{Typical procedures}

\section{Protocol A: sequential quench}

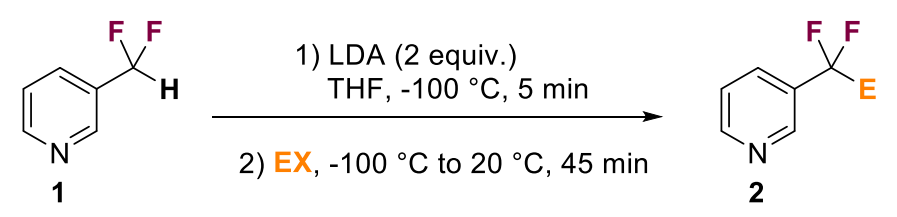

A solution of DIPA (2 equiv., $0.54 \mathrm{~mL}, 3.84 \mathrm{mmol}$ ) in THF $(7 \mathrm{~mL}$ ) under inert atmosphere and cooled to $0{ }^{\circ} \mathrm{C}$ was treated with $n$-BuLi (2 equiv., $1.6 \mathrm{M}$ in hexanes, $2.4 \mathrm{~mL}, 3.84 \mathrm{mmol}$ ). After $5 \mathrm{~min}$, the solution was cooled to $-100^{\circ} \mathrm{C}$. A solution of 3-(difluoromethyl)pyridine (1 equiv., $0.20 \mathrm{~mL}, 1.92 \mathrm{mmol}$ ) in THF $(7 \mathrm{~mL})$ pre-cooled to $-100^{\circ} \mathrm{C}$ was cannulated onto the base solution. After $5 \mathrm{~min}$, the electrophile was added in one portion to the solution. The reaction mixture was stirred for $15 \mathrm{~min}$ at $-100{ }^{\circ} \mathrm{C}$ and then slowly warmed to $20^{\circ} \mathrm{C}$ over $30 \mathrm{~min}$. Fluorobenzene was used as an internal standard in ${ }^{19} \mathrm{~F} \mathrm{NMR}$.

Protocol B: in situ quench

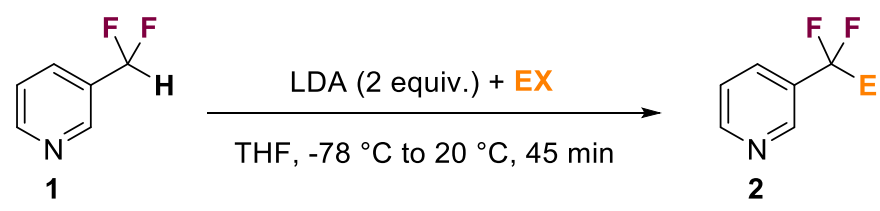

A solution of DIPA (2 equiv., $0.54 \mathrm{~mL}, 3.84 \mathrm{mmol}$ ) in THF $(7 \mathrm{~mL}$ ) under inert atmosphere and cooled to $0{ }^{\circ} \mathrm{C}$ was treated with $n$-BuLi (2 equiv., $1.6 \mathrm{M}$ in hexanes, $2.4 \mathrm{~mL}, 3.84 \mathrm{mmol}$ ). After $5 \mathrm{~min}$, the solution was cooled to $-78^{\circ} \mathrm{C}$ and the electrophile was added. A solution of 3-(difluoromethyl)pyridine (1 equiv., $0.20 \mathrm{~mL}, 1.92 \mathrm{mmol})$ in THF $(7 \mathrm{~mL})$ pre-cooled to $-78^{\circ} \mathrm{C}$ was cannulated onto the first solution. The reaction mixture was stirred for $15 \mathrm{~min}$ at $-78{ }^{\circ} \mathrm{C}$ and then slowly warmed to $20{ }^{\circ} \mathrm{C}$ over $30 \mathrm{~min}$. Fluorobenzene was used as an internal standard in ${ }^{19} \mathrm{~F}$ NMR. 


\section{Trapping of difluoro(3-pyridyl)methyllithium with electrophiles}

\section{3-(Difluoro(trimethylsilyl)methyl)pyridine (2a)}

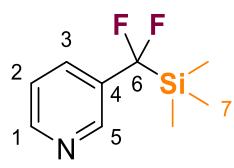

Sequential quench: $2 \mathrm{a}$ was prepared according to protocol A using TMSCl (1 equiv., $1.92 \mathrm{mmol}, 0.25$ $\mathrm{mL}) .80 \%$ of ${ }^{19} \mathrm{~F} \mathrm{NMR}$ yield was calculated with fluorobenzene as internal standard. Purification via flash column chromatography (silica gel, gradient from 0 to $40 \%$ of EtOAc in cyclohexane) yielded $2 a$ as a yellow oil (36 mg, $0.15 \mathrm{mmol}, 66 \%$ ).

In situ quench: 2a was prepared according to protocol B using TMSCl (1 equiv., $1.92 \mathrm{mmol}, 0.25 \mathrm{~mL}$ ). $77 \%$ of ${ }^{19} \mathrm{~F}$ NMR yield was calculated with fluorobenzene as internal standard.

${ }^{1} \mathrm{H}$ NMR $\left(400 \mathrm{MHz}, \mathrm{CDCl}_{3}\right): \delta 8.59-8.57(\mathrm{~m}, 1 \mathrm{H}, \mathrm{H} 1), 8.57-8.55(\mathrm{~m}, 1 \mathrm{H}, \mathrm{H} 5), 7.64-7.54(\mathrm{~m}, 1 \mathrm{H}, \mathrm{H} 3)$, $7.32-7.23(\mathrm{~m}, 1 \mathrm{H}, \mathrm{H} 2), 0.10(\mathrm{~s}, 9 \mathrm{H}, \mathrm{H} 7)$.

${ }^{13} \mathrm{C}$ NMR (101 MHz, CDCl 3$): \delta 150.2\left(\mathrm{t}, \mathrm{C}-\mathrm{F},{ }^{5} \mathrm{~J}_{\mathrm{C}-\mathrm{F}}=2.7 \mathrm{~Hz}, \mathrm{C} 1\right), 146.4\left(\mathrm{t}, \mathrm{C}-\mathrm{F},{ }^{3} \mathrm{~J}_{\mathrm{C}-\mathrm{F}}=8.2 \mathrm{~Hz}, \mathrm{C} 5\right), 133.9(\mathrm{t}$, $\left.C-F,{ }^{2} J_{C-F}=20.8 \mathrm{~Hz}, C 4\right), 132.4\left(\mathrm{t}, \mathrm{C}-\mathrm{F},{ }^{3} J_{\mathrm{C}-\mathrm{F}}=7.6 \mathrm{~Hz}, \mathrm{C} 3\right), 127.2\left(\mathrm{t}, \mathrm{C}-\mathrm{F},{ }^{1} J_{\mathrm{C}-\mathrm{F}}=265.3 \mathrm{~Hz}, \mathrm{C} 6\right), 123.1(\mathrm{C} 2),-$ $5.0\left(\mathrm{t}, \mathrm{C}-\mathrm{F},{ }^{3} \mathrm{~J}_{\mathrm{C}-\mathrm{F}}=1.8 \mathrm{~Hz}, \mathrm{C} 7\right)$.

${ }^{19} \mathrm{~F}$ NMR (376 $\left.\mathrm{MHz}, \mathrm{CDCl}_{3}\right): \delta-113.34(\mathrm{~s}, 2 \mathrm{~F})$.

${ }^{29} \mathrm{Si}$ NMR $\left(99 \mathrm{MHz}, \mathrm{CDCl}_{3}\right): \delta 5.08\left(\mathrm{t}, \mathrm{Si}-\mathrm{F},{ }^{2} J_{\mathrm{Si}-\mathrm{F}}=36.8 \mathrm{~Hz}, 1 \mathrm{Si}\right)$.

HRMS (ESI): $\mathrm{m} / \mathrm{z}[\mathrm{M}+\mathrm{H}]^{+}$calcd for $\mathrm{C}_{9} \mathrm{H}_{14} \mathrm{~F}_{2} \mathrm{NSi}$ : 202.0858; found: 202.0845.

FTIR (cm ${ }^{-1}$, neat): 2965, 1591, 1577, 1478, 1418, 1246, 1081, 991, 839, 803, 754, 715, 704. 
${ }^{1} \mathrm{H}$ NMR $\left(400 \mathrm{MHz}, \mathrm{CDCl}_{3}\right.$ ) of 2a:

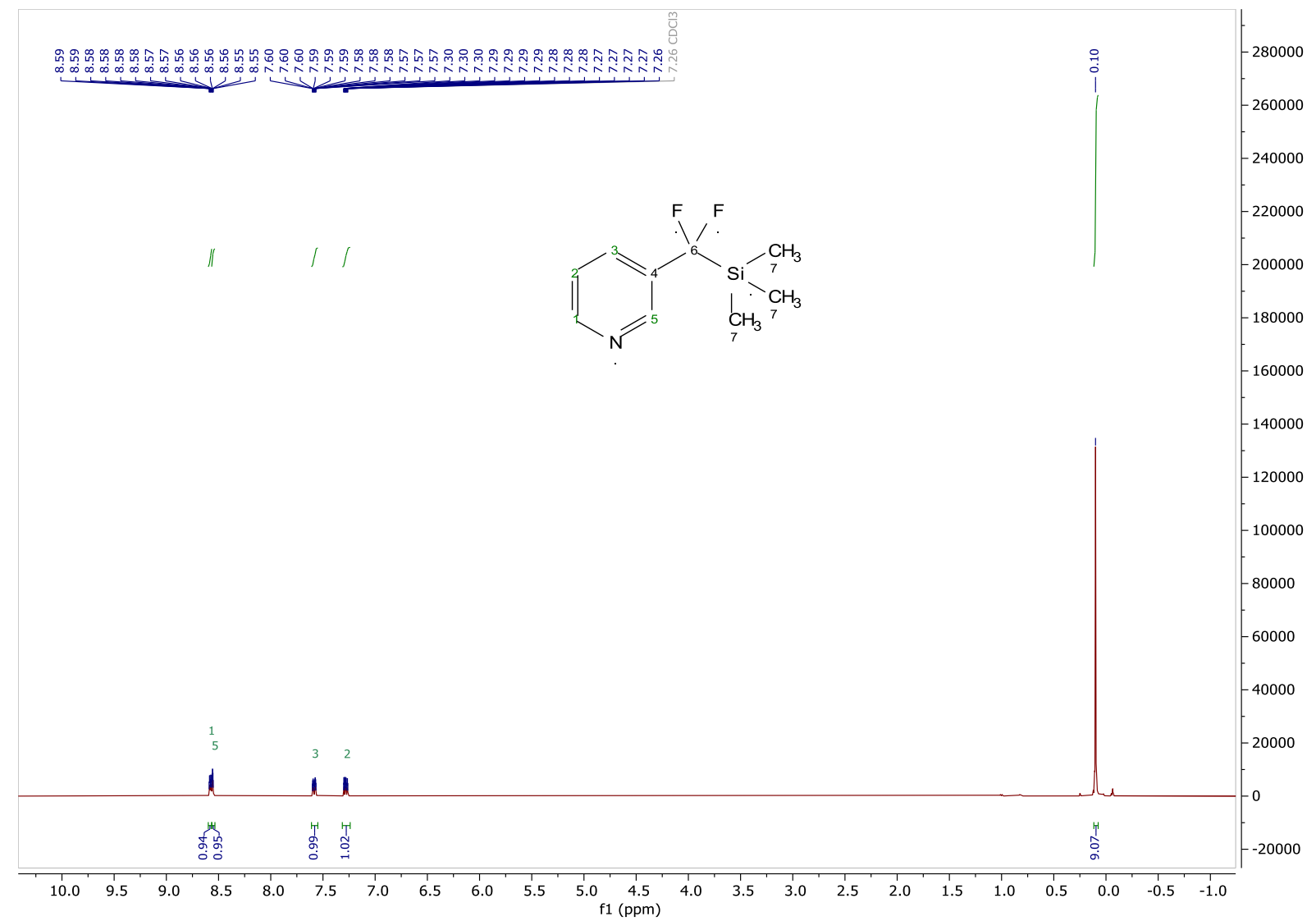

${ }^{13} \mathrm{C}$ NMR (101 MHz, $\left.\mathrm{CDCl}_{3}\right)$ of 2a:

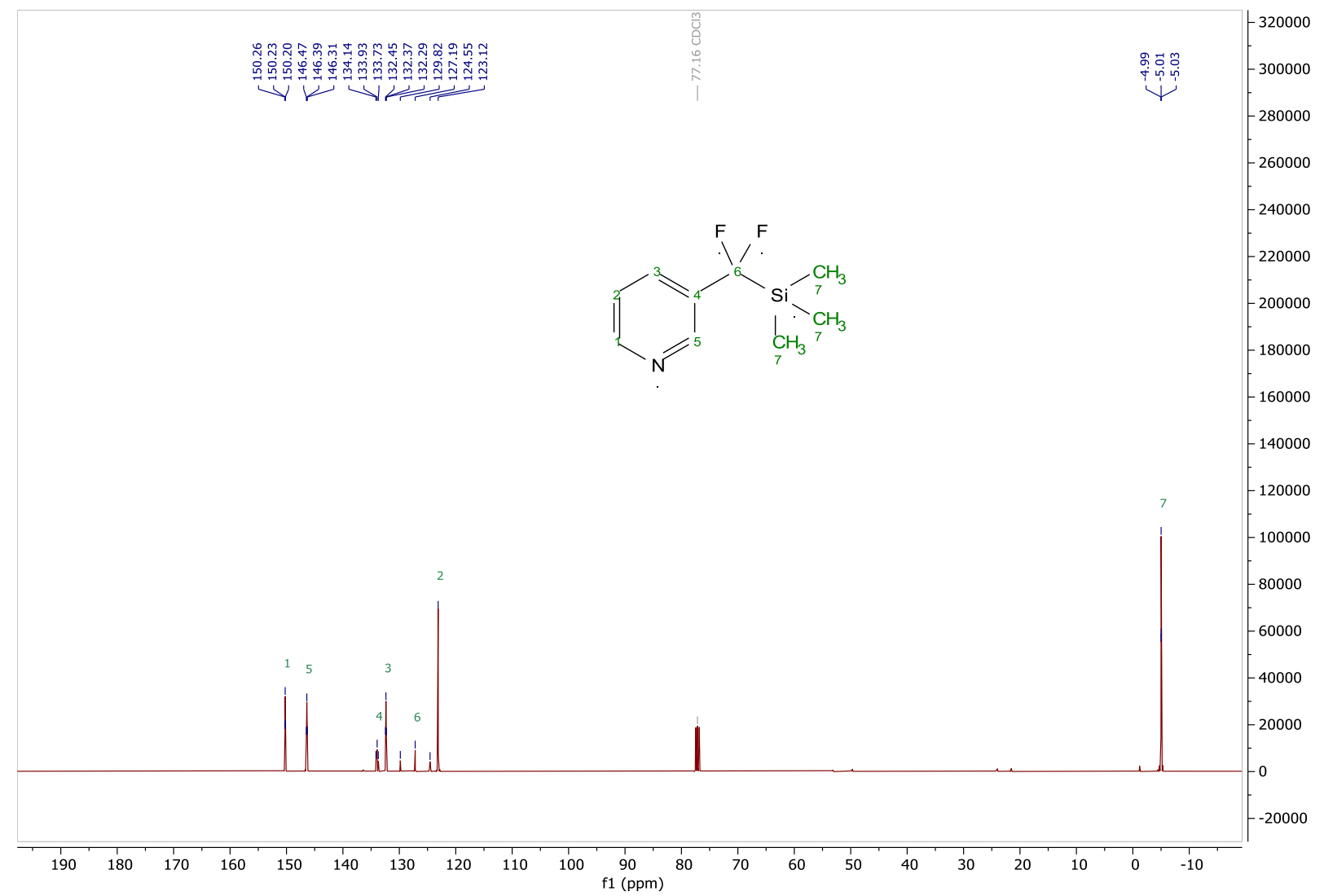


${ }^{19} \mathrm{~F} \mathrm{NMR} \mathrm{(376} \mathrm{MHz,} \mathrm{CDCl}_{3}$ ) of 2a:

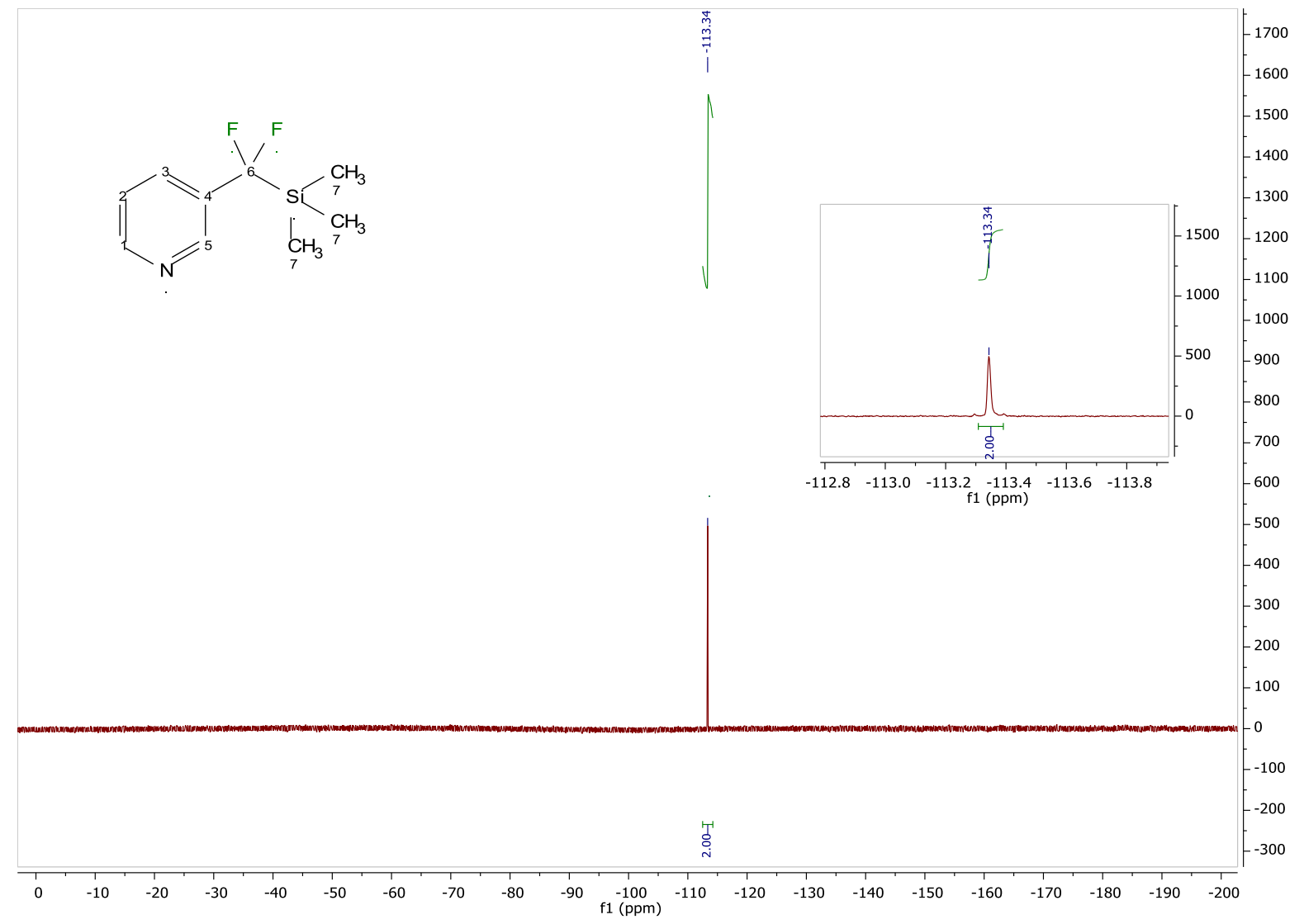

${ }^{29} \mathrm{Si} \mathrm{NMR} \mathrm{(99} \mathrm{MHz,} \mathrm{CDCl}_{3}$ ) of 2a:

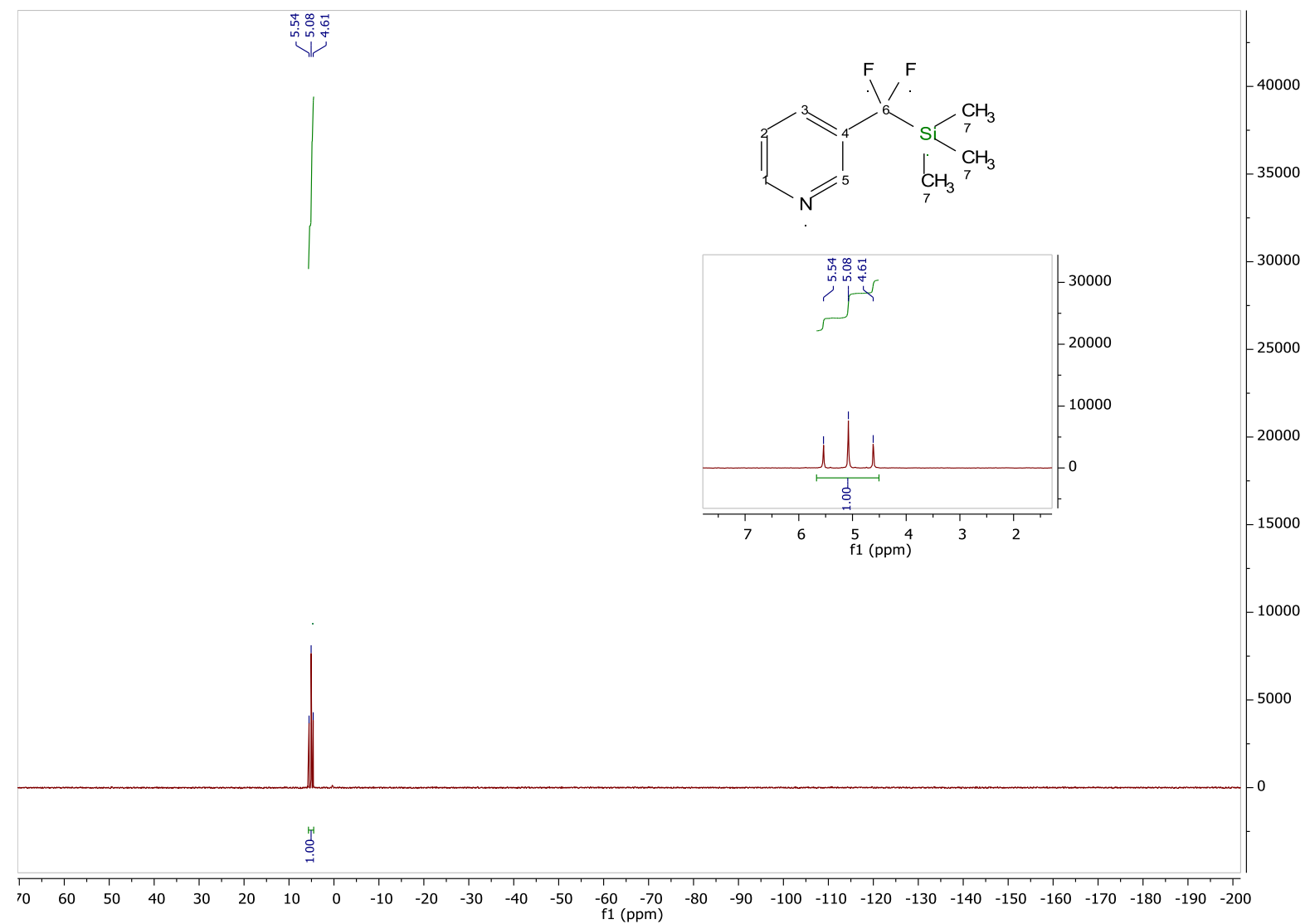




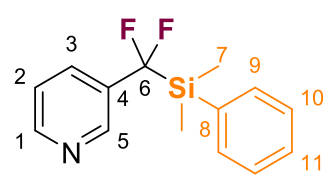

Sequential quench: $\mathbf{2} \mathbf{b}$ was prepared according to protocol $\mathbf{A}$ using chlorodimethylphenylsilane (2 equiv., $3.84 \mathrm{mmol}, 0.64 \mathrm{~mL}$ ). $77 \%$ of ${ }^{19} \mathrm{~F} \mathrm{NMR}$ yield was calculated with fluorobenzene as internal standard. Purification via flash column chromatography (silica gel, gradient from 0 to $40 \%$ of EtOAc in cyclohexane) yielded $\mathbf{2 b}$ as an orange oil (355 mg, $1.35 \mathrm{mmol}, 70 \%)$.

In situ quench: $\mathbf{2 b}$ was prepared according to protocol B using chlorodimethylphenylsilane (2 equiv., $3.84 \mathrm{mmol}, 0.64 \mathrm{~mL}$ ). $76 \%$ of ${ }^{19} \mathrm{~F} \mathrm{NMR}$ yield was calculated with fluorobenzene as internal standard.

${ }^{1} \mathrm{H}$ NMR $\left(500 \mathrm{MHz}, \mathrm{CDCl}_{3}\right): \delta 8.56(\mathrm{~d}, J=4.7 \mathrm{~Hz}, 1 \mathrm{H}, \mathrm{H1}), 8.39(\mathrm{~d}, J=1.2 \mathrm{~Hz}, 1 \mathrm{H}, \mathrm{H} 5), 7.46-7.30(\mathrm{~m}, 6 \mathrm{H}$, $\mathrm{H} 3, \mathrm{H} 9, \mathrm{H} 10, \mathrm{H} 11), 7.19$ (dd, J = 8.0, $4.8 \mathrm{~Hz}, 1 \mathrm{H}, \mathrm{H} 2), 0.47$ (s, 6H, H7).

${ }^{13} \mathrm{C}$ NMR $\left(126 \mathrm{MHz}, \mathrm{CDCl}_{3}\right): \delta 150.2\left(\mathrm{t}, \mathrm{C}-\mathrm{F},{ }^{5} \mathrm{~J}_{\mathrm{C}-\mathrm{F}}=2.7 \mathrm{~Hz}, \mathrm{C} 1\right), 146.5\left(\mathrm{t}, \mathrm{C}-\mathrm{F},{ }^{3} \mathrm{~J}_{\mathrm{C}-\mathrm{F}}=8.1 \mathrm{~Hz}, \mathrm{C} 5\right), 134.5\left(\mathrm{C}_{\mathrm{Ar}}\right)$, $133.5\left(\mathrm{t}, \mathrm{C}-\mathrm{F},{ }^{2} \mathrm{~J}_{\mathrm{C}-\mathrm{F}}=20.8 \mathrm{~Hz}, \mathrm{C} 4\right), 132.7\left(\mathrm{t}, \mathrm{C}-\mathrm{F},{ }^{3} \mathrm{~J}_{\mathrm{C}-\mathrm{F}}=7.5 \mathrm{~Hz}, \mathrm{C} 3\right), 131.6(\mathrm{C} 8), 130.6\left(\mathrm{C}_{\mathrm{Ar}}\right), 128.2\left(\mathrm{C}_{\mathrm{Ar}}\right), 126.9$ $\left(\mathrm{t}, \mathrm{C}-\mathrm{F},{ }^{1} \mathrm{~J}_{\mathrm{C}-\mathrm{F}}=266.0 \mathrm{~Hz}, \mathrm{C} 6\right), 122.9(\mathrm{C} 2),-6.7$ (C7).

${ }^{19} \mathrm{~F}$ NMR (471 MHz, $\left.\mathrm{CDCl}_{3}\right): \delta-111.61(\mathrm{~s}, 2 \mathrm{~F})$.

${ }^{29}$ Si NMR (99 MHz, CDCl 3 ): $\delta-2.87\left(\mathrm{t}, \mathrm{Si}-\mathrm{F},{ }^{2} \mathrm{~J}_{\mathrm{Si}-\mathrm{F}}=36.9 \mathrm{~Hz}, 1 \mathrm{Si}\right)$.

HRMS (ESI): $\mathrm{m} / \mathrm{z}[\mathrm{M}+\mathrm{H}]^{+}$calcd for $\mathrm{C}_{14} \mathrm{H}_{16} \mathrm{~F}_{2} \mathrm{NSi}$ : 264.1015; found: 264.1007 .

FTIR (cm ${ }^{-1}$, neat): $3072,2963,1591,1580,1478,1428,1418,1248,1117,1077,993,837,806,785$, 737, 707, 698. 
${ }^{1} \mathrm{H}$ NMR $\left(500 \mathrm{MHz}, \mathrm{CDCl}_{3}\right.$ ) of $2 \mathrm{~b}$ :

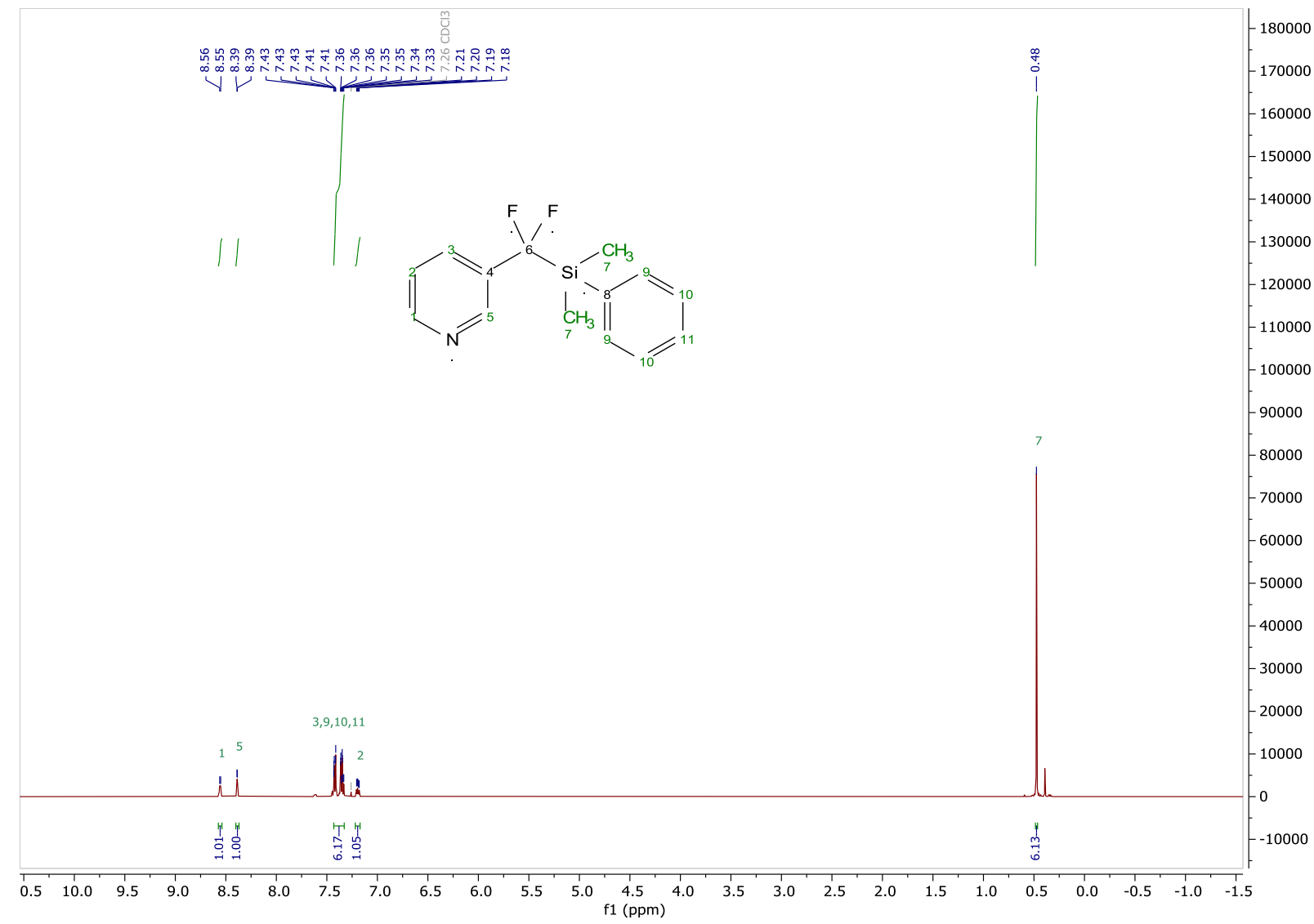

${ }^{13} \mathrm{C}$ NMR $\left(126 \mathrm{MHz}, \mathrm{CDCl}_{3}\right)$ of $2 \mathrm{~b}$ :

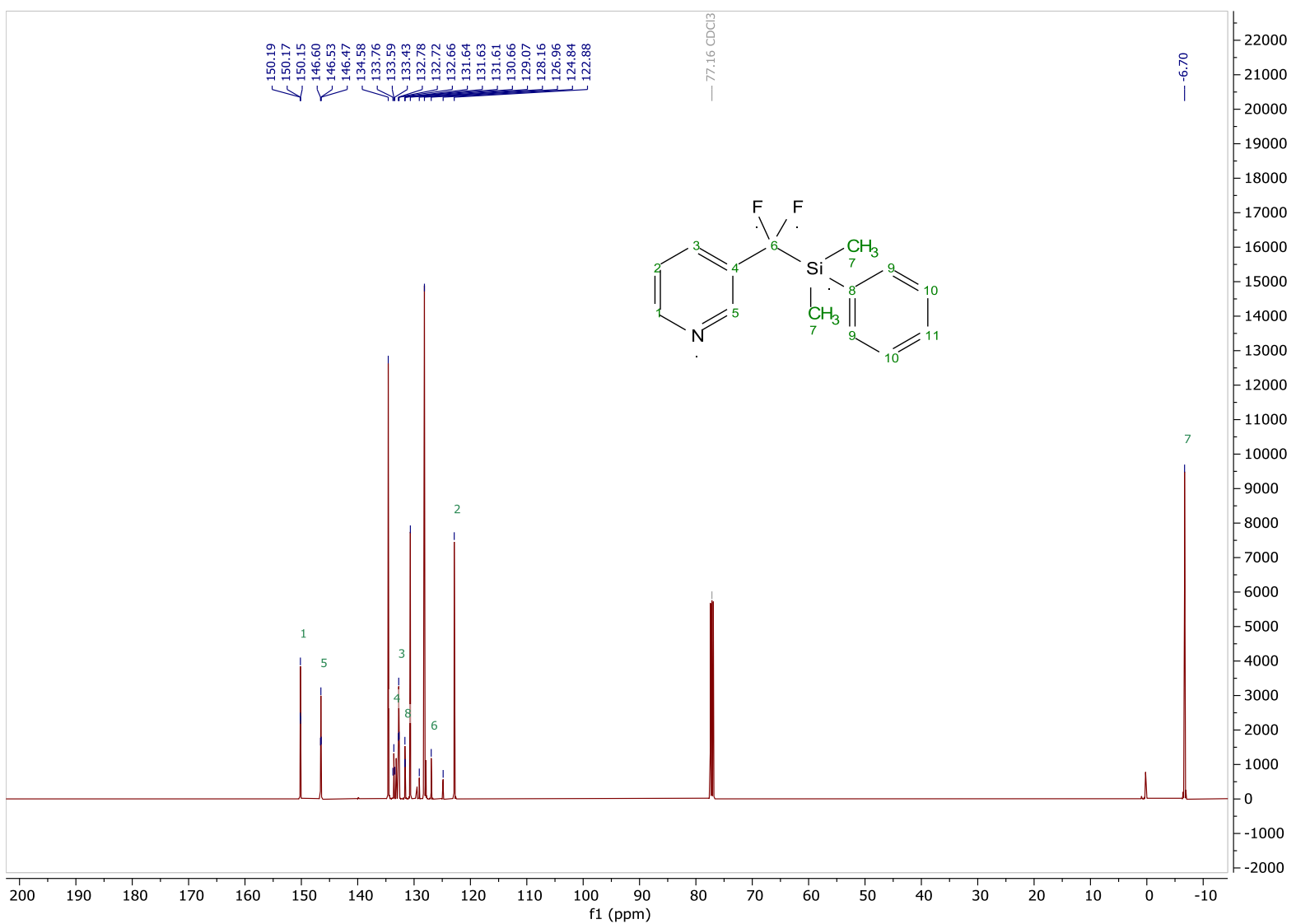


${ }^{19} \mathrm{~F} \mathrm{NMR} \mathrm{(471} \mathrm{MHz,} \mathrm{CDCl}_{3}$ ) of $2 \mathrm{~b}$ :

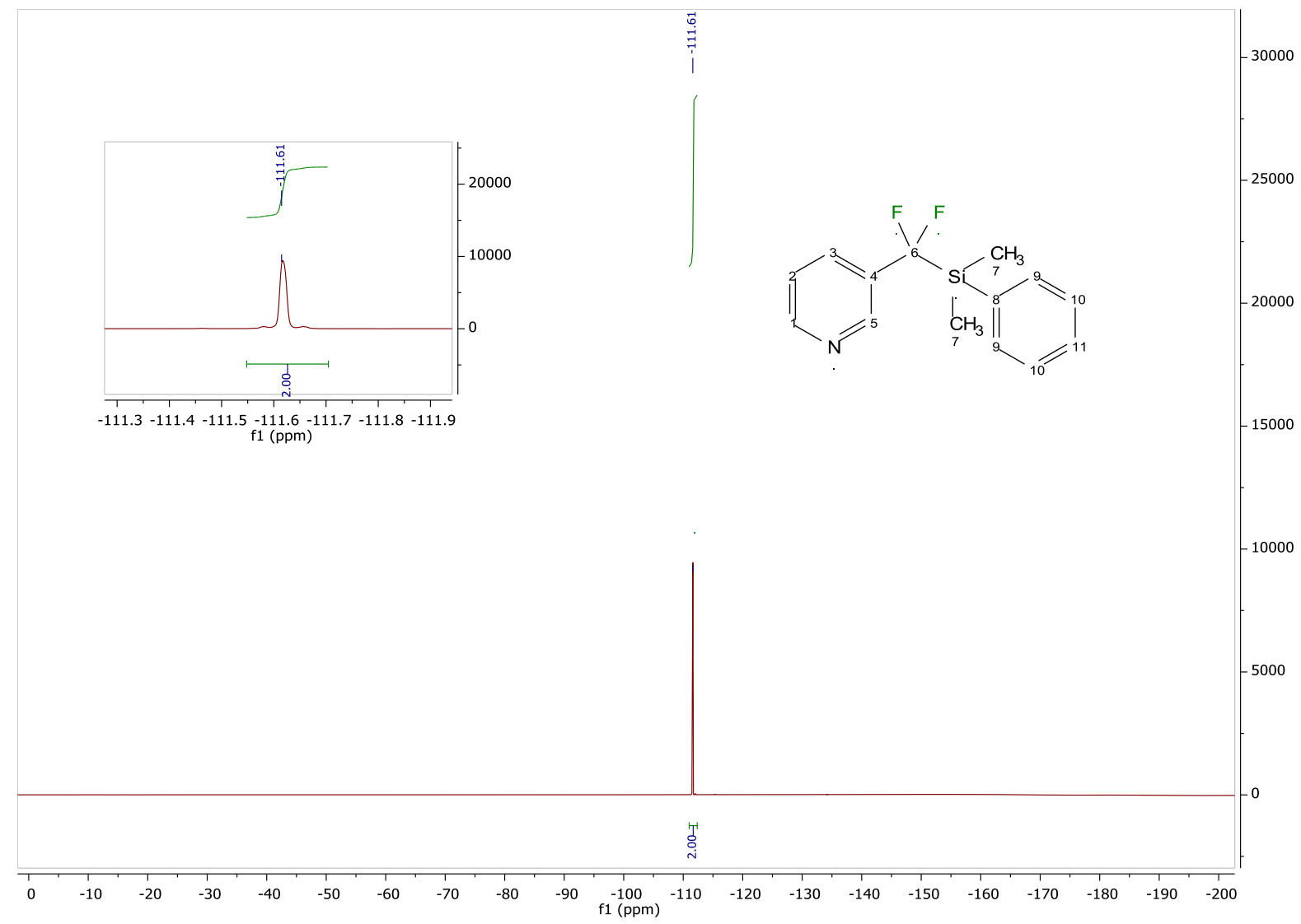

${ }^{29} \mathrm{Si} \mathrm{NMR} \mathrm{(99} \mathrm{MHz,} \mathrm{CDCl}_{3}$ ) of $2 \mathrm{~b}$ :

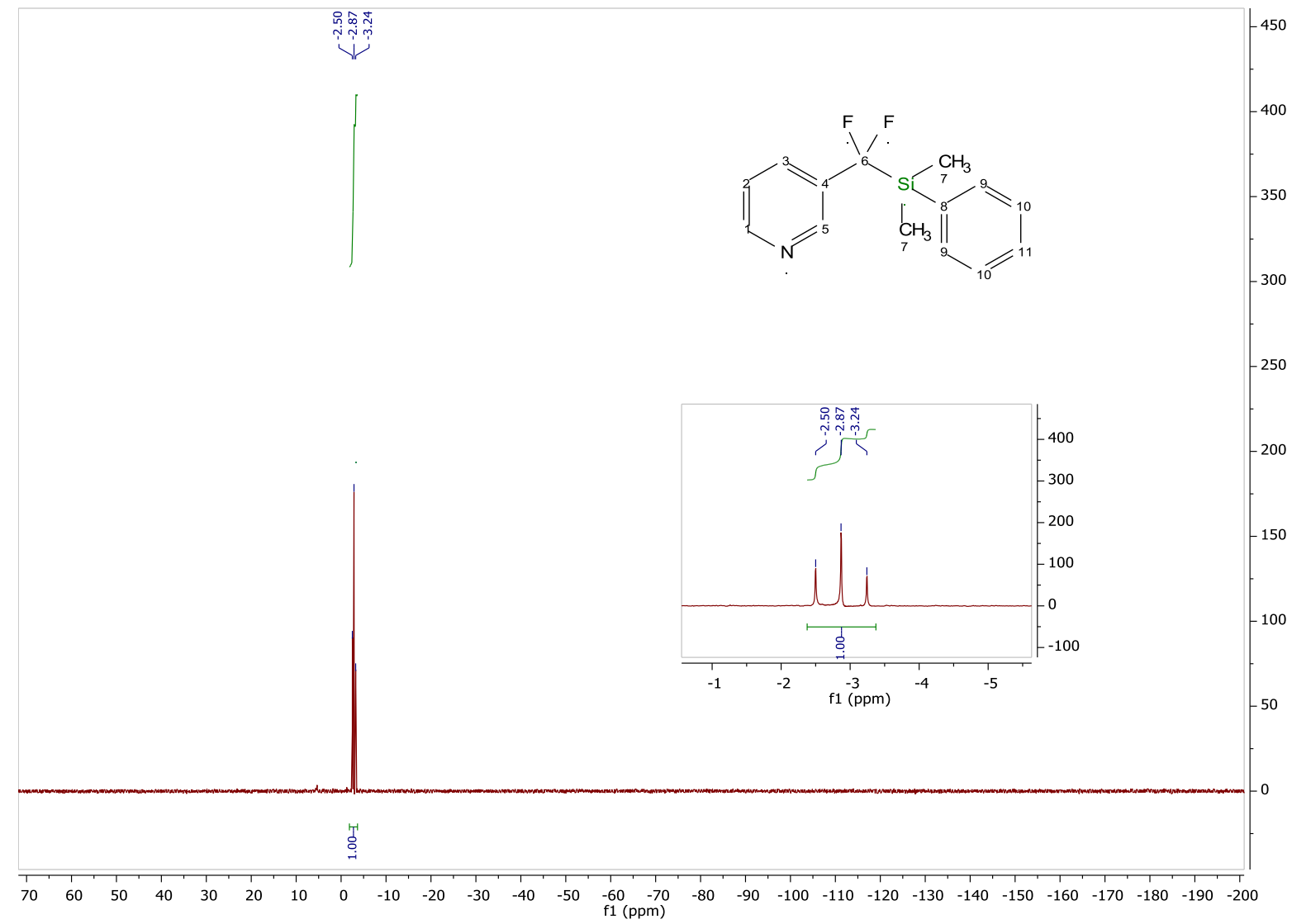


<smiles>CC(C)(C)[Si](C)(C)C(F)(F)c1cccnc1</smiles>

Sequential quench: $\mathbf{2 c}$ was prepared according to protocol A using tert-butyldimethylsilyl chloride (2 equiv., $3.84 \mathrm{mmol}, 579 \mathrm{mg}$ ). $21 \%$ of ${ }^{19} \mathrm{~F} \mathrm{NMR}$ yield was calculated with fluorobenzene as internal standard. Purification via flash column chromatography (silica gel, gradient from 0 to $30 \%$ of EtOAc in cyclohexane) yielded $\mathbf{2 c}$ as a yellow oil ( $70 \mathrm{mg}, 0.29 \mathrm{mmol}, 15 \%)$.

In situ quench: $2 \mathrm{c}$ was prepared according to protocol B using tert-butyldimethylsilyl chloride (2 equiv., $3.84 \mathrm{mmol}, 579 \mathrm{mg}$ ). $23 \%$ of ${ }^{19} \mathrm{~F}$ NMR yield was calculated with fluorobenzene as internal standard.

${ }^{1} \mathrm{H}$ NMR $\left(500 \mathrm{MHz}, \mathrm{CDCl}_{3}\right): \delta 8.65-8.58(\mathrm{~m}, 2 \mathrm{H}, \mathrm{H} 1, \mathrm{H} 5), 7.70-7.62(\mathrm{~m}, 1 \mathrm{H}, \mathrm{H} 3), 7.34$ (ddd, J = 7.8, 4.8, $0.9 \mathrm{~Hz}, 1 \mathrm{H}, \mathrm{H} 2), 0.95(\mathrm{~s}, 9 \mathrm{H}, \mathrm{H} 9), 0.09(\mathrm{~s}, 6 \mathrm{H}, \mathrm{H} 7)$.

${ }^{13} \mathrm{C}$ NMR (126 MHz, CDCl $)$ : $\delta 150.2\left(\mathrm{t}, \mathrm{C}-\mathrm{F},{ }^{5} \mathrm{~J}_{\mathrm{C}-\mathrm{F}}=2.8 \mathrm{~Hz}, \mathrm{C} 1\right), 146.6\left(\mathrm{t}, \mathrm{C}-\mathrm{F},{ }^{3} \mathrm{~J}_{\mathrm{C}-\mathrm{F}}=8.4 \mathrm{~Hz}, \mathrm{C} 5\right), 134.9(\mathrm{t}$, $\left.\mathrm{C}-\mathrm{F},{ }^{2} J_{\mathrm{C}-\mathrm{F}}=20.9 \mathrm{~Hz}, \mathrm{C} 4\right), 132.7\left(\mathrm{t}, \mathrm{C}-\mathrm{F},{ }^{3} J_{\mathrm{C}-\mathrm{F}}=7.8 \mathrm{~Hz}, \mathrm{C} 3\right), 128.3\left(\mathrm{t}, \mathrm{C}-\mathrm{F},{ }^{1} J_{\mathrm{C}-\mathrm{F}}=266.7 \mathrm{~Hz}, \mathrm{C} 6\right), 123.2(\mathrm{C} 2)$, 26.8 (C9), 17.2 (C8), -8.2 (C7).

${ }^{19}$ F NMR (471 MHz, $\left.\mathrm{CDCl}_{3}\right): \delta-107.83(\mathrm{~s}, 2 \mathrm{~F})$.

${ }^{29} \mathrm{Si} \mathrm{NMR}\left(99 \mathrm{MHz}, \mathrm{CDCl}_{3}\right): \delta 8.03\left(\mathrm{t}, \mathrm{Si}-\mathrm{F},{ }^{2} \mathrm{~J}_{\mathrm{Si}-\mathrm{F}}=33.5 \mathrm{~Hz}, 1 \mathrm{Si}\right)$.

HRMS (ESI): $\mathrm{m} / \mathrm{z}[\mathrm{M}+\mathrm{H}]^{+}$calcd for $\mathrm{C}_{12} \mathrm{H}_{20} \mathrm{~F}_{2} \mathrm{NSi}$ : 244.1328; found: 244.1332 .

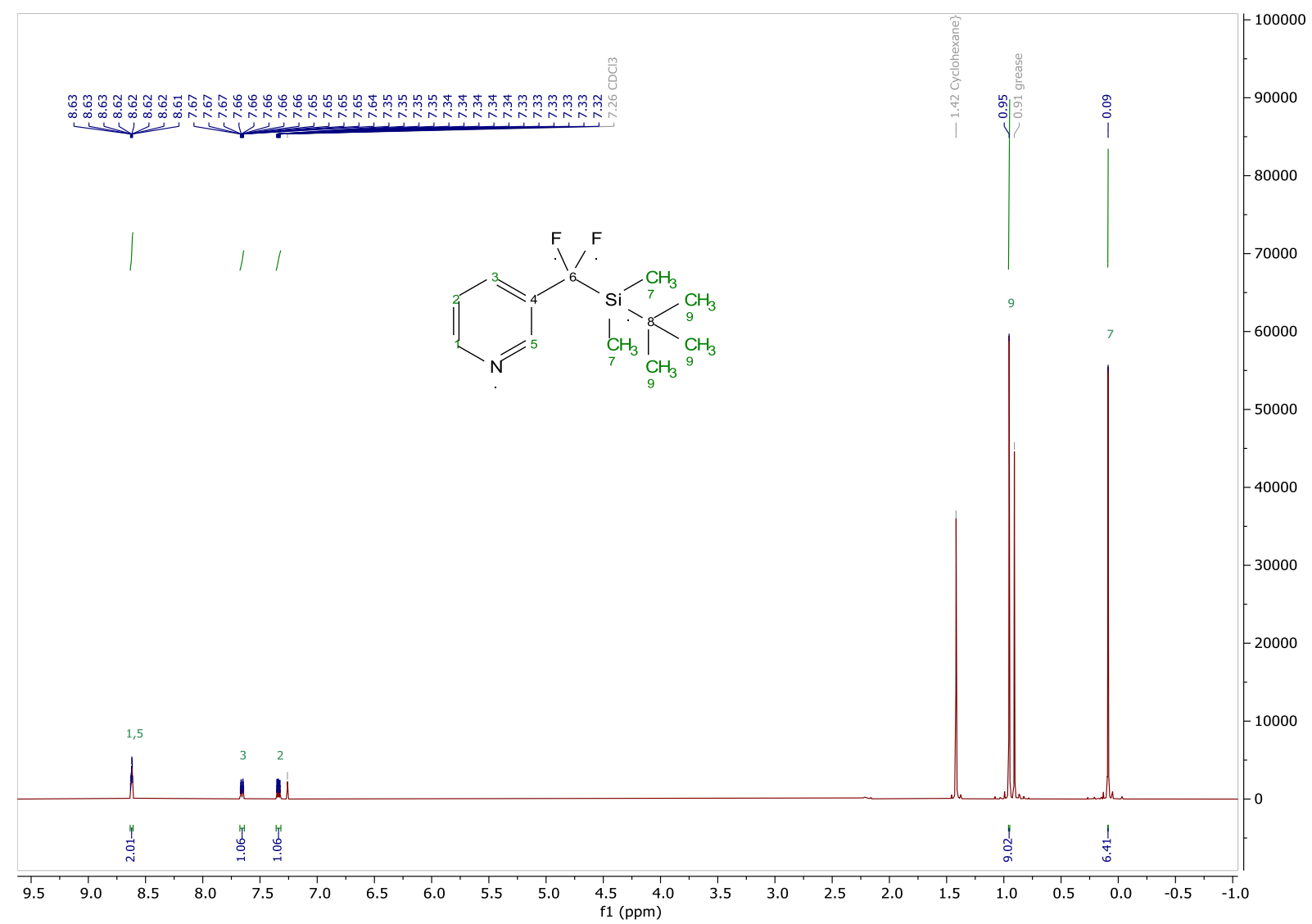


${ }^{13} \mathrm{C}$ NMR $\left(126 \mathrm{MHz}, \mathrm{CDCl}_{3}\right)$ of 2c:

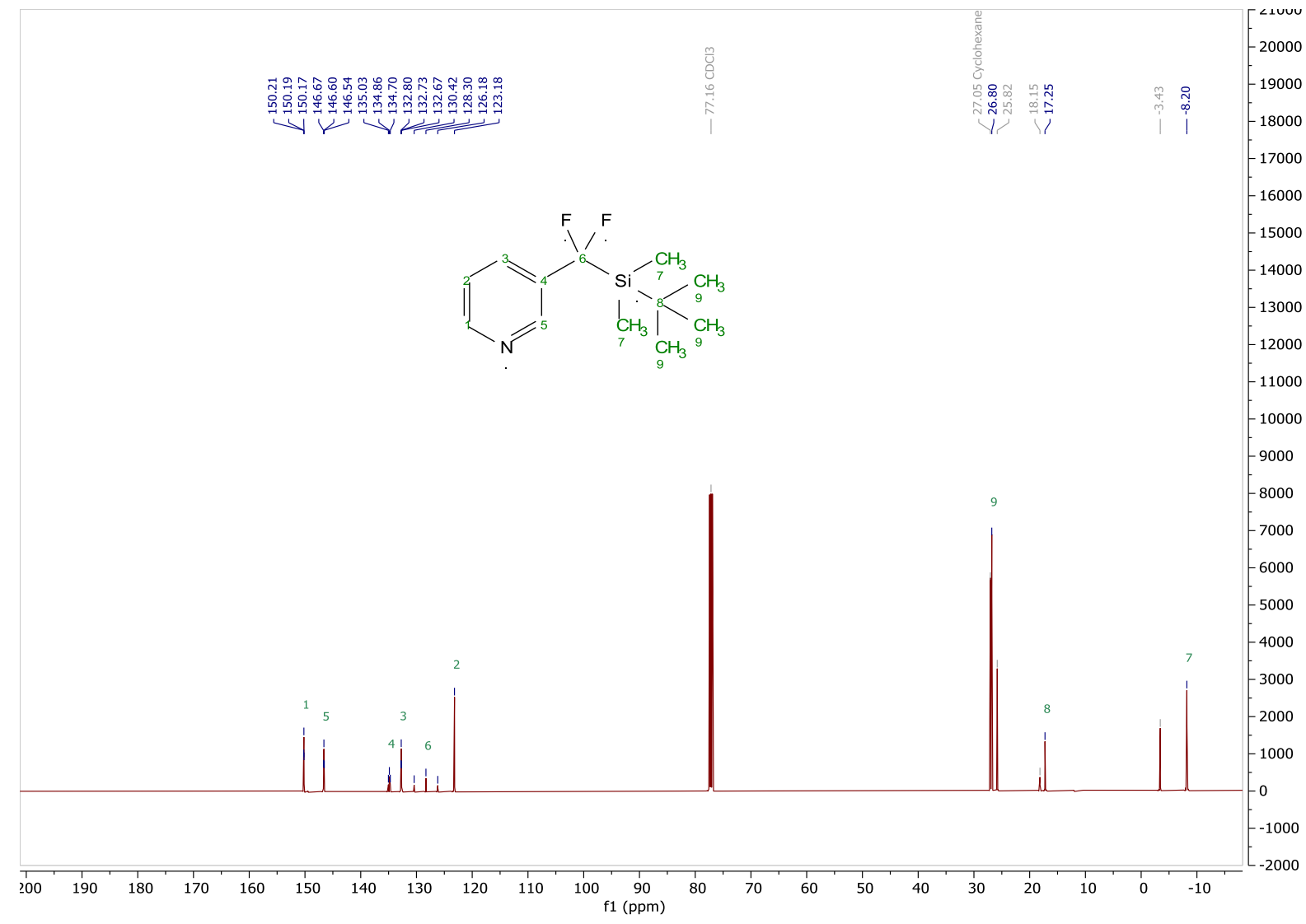

${ }^{19} \mathrm{~F}$ NMR (471 MHz, $\mathrm{CDCl}_{3}$ ) of 2c:

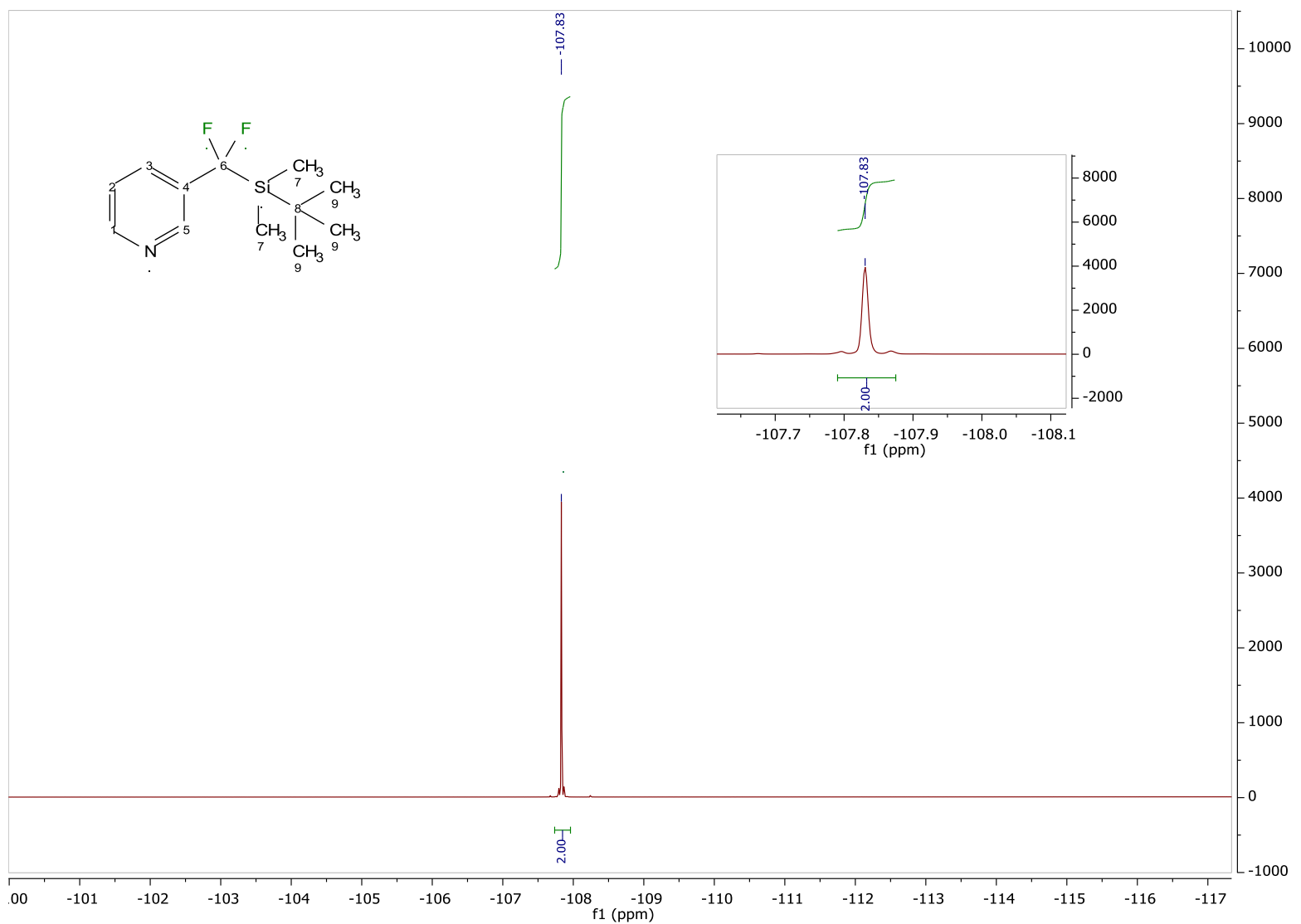


${ }^{29} \mathrm{Si} \mathrm{NMR} \mathrm{(99} \mathrm{MHz,} \mathrm{CDCl}_{3}$ ) of 2c:

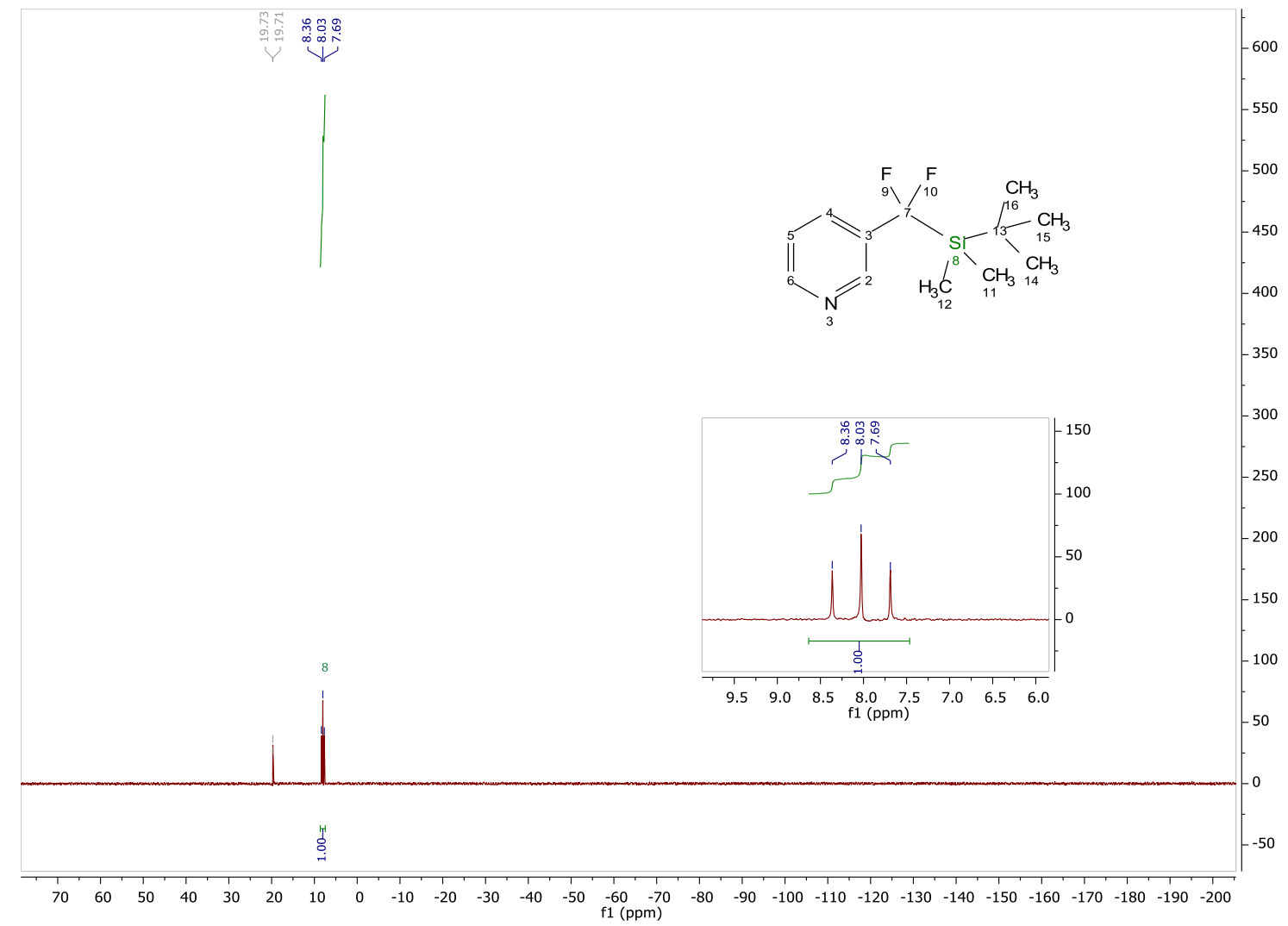




\section{3-(Difluoromethyl-d)pyridine (2d)}<smiles>[2H]C(F)(F)c1cccnc1</smiles>

Sequential quench: $\mathbf{2} \mathbf{d}$ was prepared according to protocol $\mathbf{A}$ using $\mathrm{DCl}$ previously prepared in situ by mixing TMSCl (1 equiv., $1.92 \mathrm{mmol}, 0.25 \mathrm{~mL}$ ) and $d$-Methanol (12.7 equiv. $24.51 \mathrm{mmol}, 1 \mathrm{~mL}$ ). $49 \%$ of ${ }^{19} \mathrm{~F}$ NMR yield was calculated with fluorobenzene as internal standard. Purification from the starting material (protonated analogue) was not possible.

${ }^{19}$ F NMR (471 MHz, $\left.\mathrm{CDCl}_{3}\right): \delta-113.17(\mathrm{t}, J=8.4 \mathrm{~Hz}, 2 \mathrm{~F})$.

${ }^{19} \mathrm{~F}$ NMR (471 MHz, $\mathrm{CDCl}_{3}$ ) of $2 \mathrm{~d}$ :

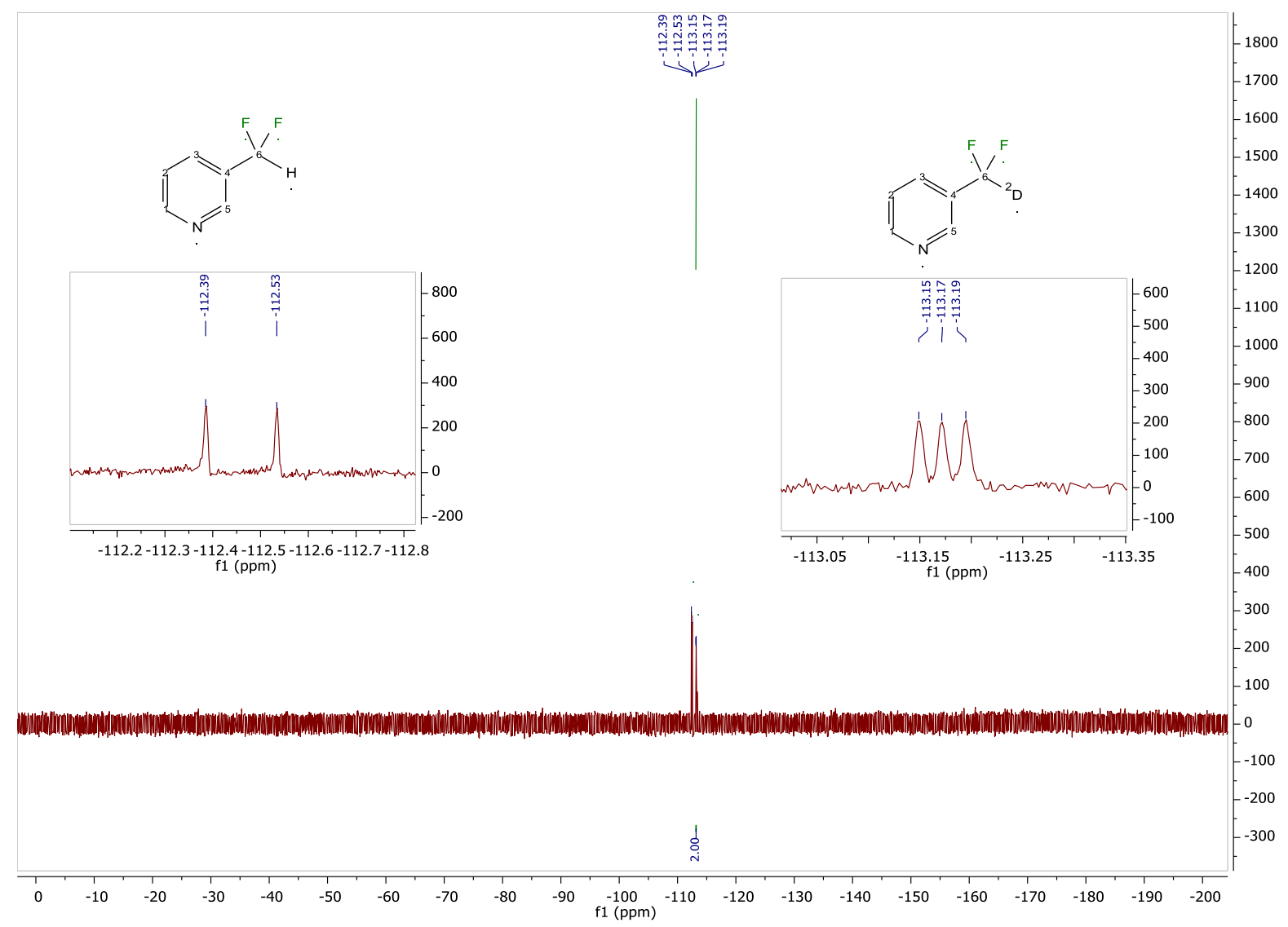

\section{3-(1,1-Difluoroethyl)pyridine (2e)}<smiles>CC(F)(F)c1cccnc1</smiles>

Sequential quench: $\mathbf{2 e}$ was prepared according to protocol A using iodomethane (1 equiv., $1.92 \mathrm{mmol}$, $0.12 \mathrm{~mL}$ ). $63 \%$ of ${ }^{19} \mathrm{~F}$ NMR yield was calculated with fluorobenzene as internal standard. Purification via flash column chromatography (silica gel, gradient from 0 to $40 \%$ of EtOAc in cyclohexane) yielded $2 e$ as a yellowish oil (60 $\mathrm{mg}, 0.42 \mathrm{mmol}, 22 \%$ ). 
${ }^{1} \mathrm{H}$ NMR (500 MHz, $\left.\mathrm{CDCl}_{3}\right): \delta 8.79-8.75(\mathrm{~m}, 1 \mathrm{H}, \mathrm{H} 5), 8.71-8.64(\mathrm{~m}, 1 \mathrm{H}, \mathrm{H} 1), 7.83-7.79(\mathrm{~m}, 1 \mathrm{H}, \mathrm{H} 3)$, 7.36 (ddd, $J=8.0,4.9,0.7 \mathrm{~Hz}, 1 \mathrm{H}, \mathrm{H} 2), 1.95$ (t, $J=18.3 \mathrm{~Hz}, 3 \mathrm{H}, \mathrm{H} 7$ ).

${ }^{13} \mathrm{C}$ NMR $\left(126 \mathrm{MHz}, \mathrm{CDCl}_{3}\right): \delta 151.2\left(\mathrm{t}, \mathrm{C}-\mathrm{F},{ }^{5} \mathrm{~J}_{\mathrm{C}-\mathrm{F}}=1.8 \mathrm{~Hz}, \mathrm{C} 1\right), 146.5\left(\mathrm{t}, \mathrm{C}-\mathrm{F},{ }^{3} \mathrm{~J}_{\mathrm{C}-\mathrm{F}}=6.4 \mathrm{~Hz}, \mathrm{C} 5\right), 133.9(\mathrm{t}$, $\left.C-F,{ }^{2} J_{C-F}=27.1 \mathrm{~Hz}, C 4\right), 132.6\left(t, C-F,{ }^{3} J_{C-F}=5.7 \mathrm{~Hz}, C 3\right), 123.4(C 2), 121.0\left(t, C-F,{ }^{1} J_{C-F}=239.4 \mathrm{~Hz}, C 6\right)$, $25.9\left(\mathrm{t}, \mathrm{C}-\mathrm{F},{ }^{2} J_{\mathrm{C}-\mathrm{F}}=29.4 \mathrm{~Hz}, \mathrm{C7}\right)$.

${ }^{19} \mathrm{~F}$ NMR (471 MHz, $\left.\mathrm{CDCl}_{3}\right): \delta-88.19\left(\mathrm{q}, \mathrm{F}-\mathrm{H},{ }^{3} \mathrm{~F}_{\mathrm{F}-\mathrm{H}}=18.3 \mathrm{~Hz}\right)$.

HRMS (ESI): $\mathrm{m} / \mathrm{z}[\mathrm{M}+\mathrm{H}]^{+}$calcd for $\mathrm{C}_{7} \mathrm{H}_{8} \mathrm{~F}_{2} \mathrm{~N}$ : 144.0619; found: 144.0613 .

These data are in accordance with previously reported results. ${ }^{4}$

${ }^{1} \mathrm{H}$ NMR $\left(500 \mathrm{MHz}, \mathrm{CDCl}_{3}\right.$ ) of $2 \mathrm{e}$ :

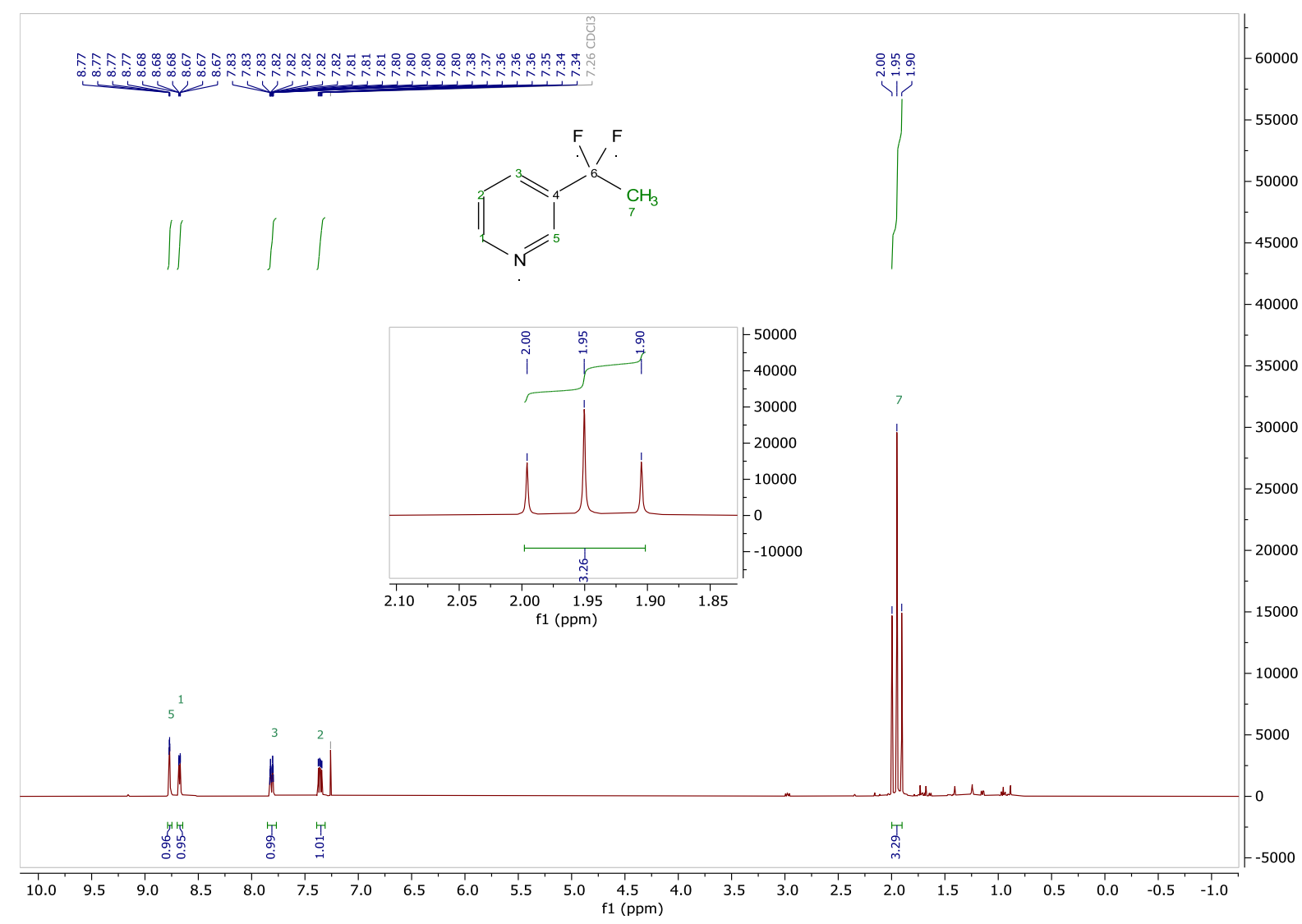


${ }^{13} \mathrm{C}$ NMR (126 MHz, $\left.\mathrm{CDCl}_{3}\right)$ of 2e:

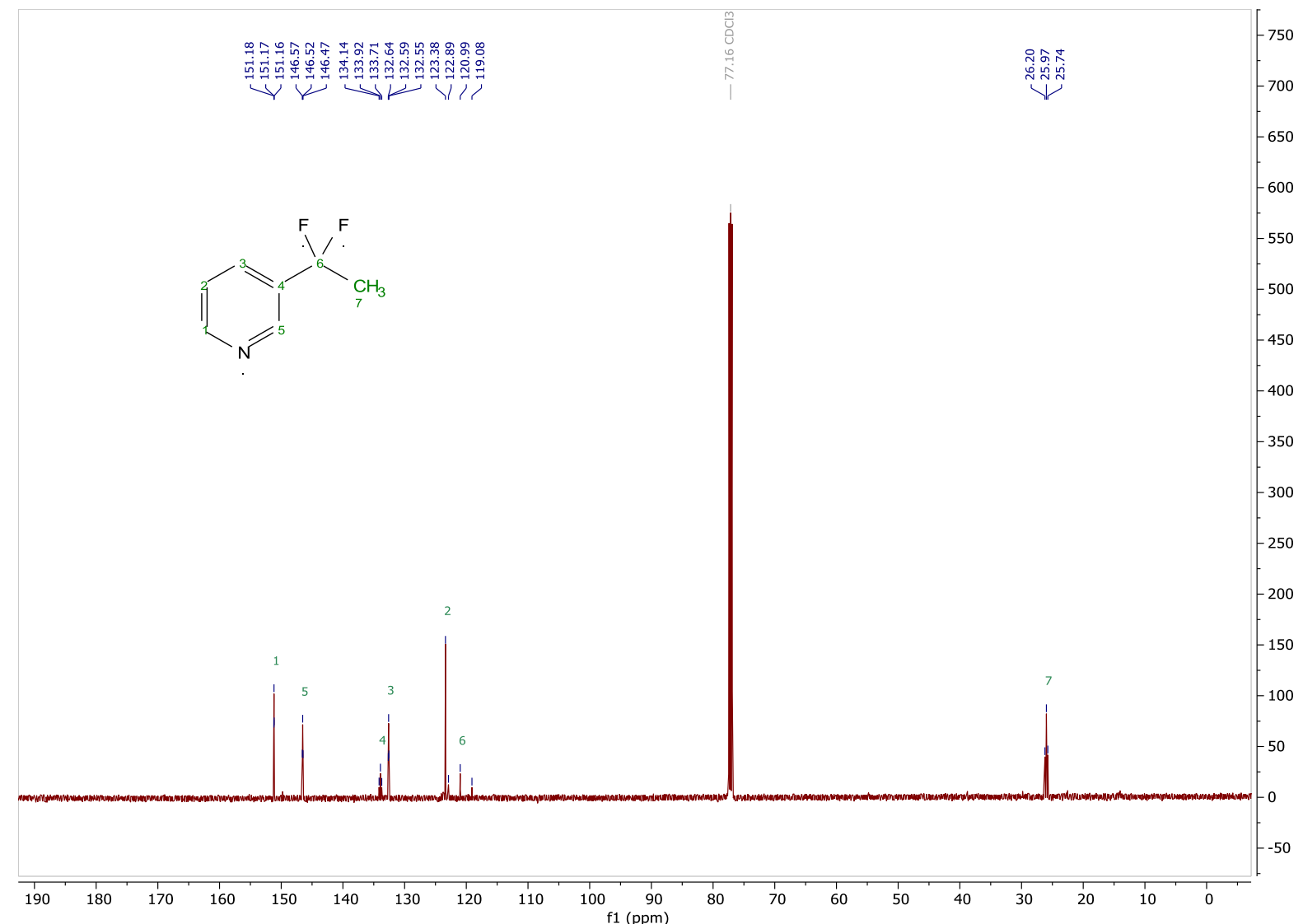

${ }^{19} \mathrm{~F}$ NMR (471 MHz, $\mathrm{CDCl}_{3}$ ) of 2e:

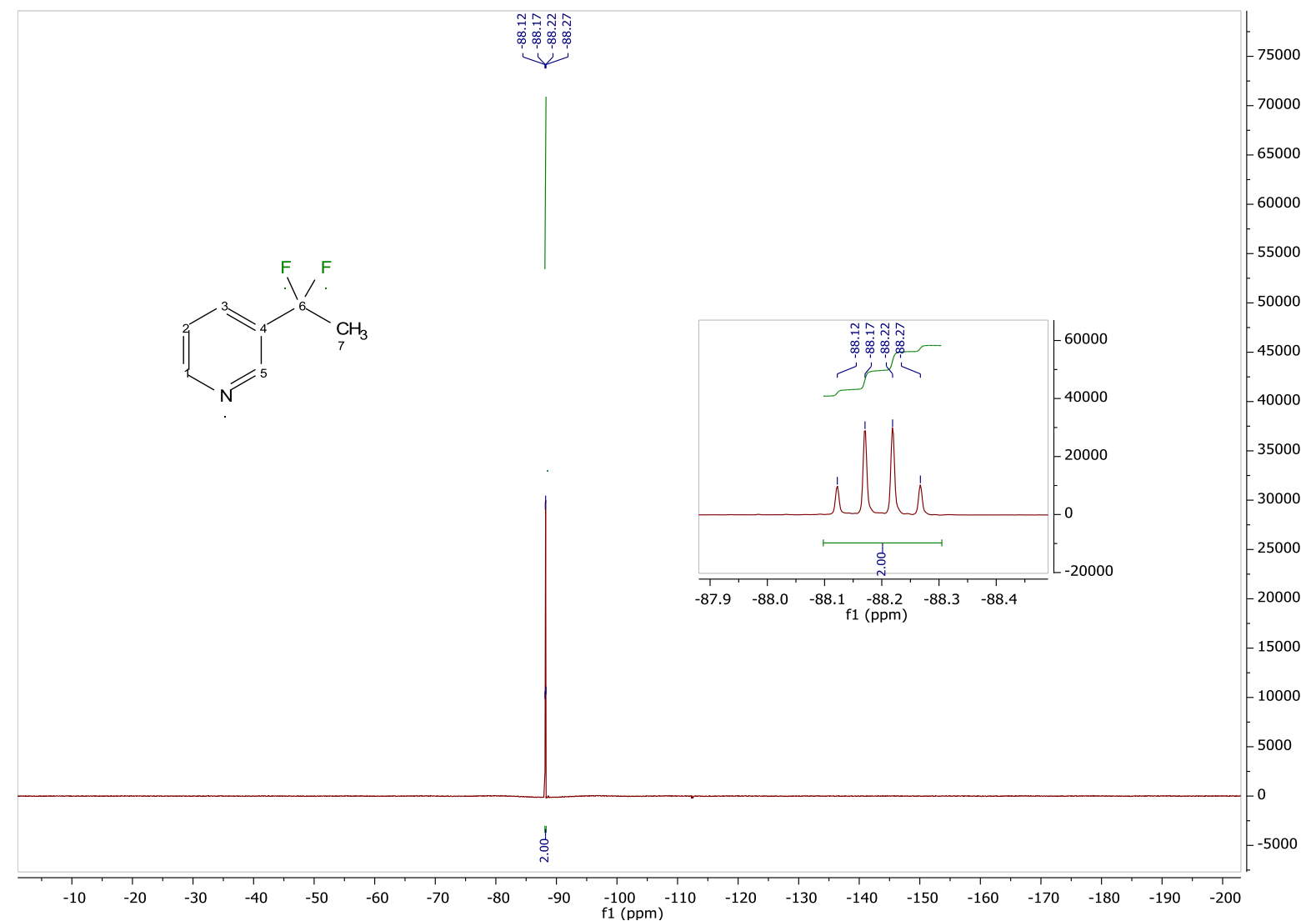




\section{3-(1,1-Difluoropentyl)pyridine (2f)}<smiles>CCCCC(F)(F)c1cccnc1</smiles>

Sequential quench: $\mathbf{2 f}$ was prepared according to protocol A using 1-iodobutane (5 equiv., $9.62 \mathrm{mmol}$, $1.1 \mathrm{~mL}$ ). $41 \%$ of ${ }^{19} \mathrm{~F}$ NMR yield was calculated with fluorobenzene as internal standard. Purification via flash column chromatography (silica gel, gradient from 0 to $20 \%$ of EtOAc in cyclohexane) yielded $\mathbf{2} f$ as a yellowish oil ( $25 \mathrm{mg}, 0.13 \mathrm{mmol}, 7 \%$ ).

${ }^{1} \mathrm{H}$ NMR (500 MHz, CDCl $)$ ): $\delta 8.73(\mathrm{~d}, J=1.0 \mathrm{~Hz}, 1 \mathrm{H}, \mathrm{H} 5), 8.68(\mathrm{~d}, J=4.6 \mathrm{~Hz}, 1 \mathrm{H}, \mathrm{H1}), 7.78(\mathrm{dt}, J=8.1$, $2.0 \mathrm{~Hz}, 1 \mathrm{H}, \mathrm{H} 3$ ), 7.36 (dd, J = 8.0, 4.8, H2), $2.21-2.06(\mathrm{~m}, 2 \mathrm{H}, \mathrm{H} 7), 1.46-1.30(\mathrm{~m}, 4 \mathrm{H}, \mathrm{H} 8, \mathrm{H} 9), 0.89$ (t, $J=7.2 \mathrm{~Hz}, 3 \mathrm{H}, \mathrm{H} 10)$.

${ }^{13} \mathrm{C}$ NMR (126 MHz, CDCl $): \delta 151.0(\mathrm{C} 1), 146.9\left(\mathrm{t}, \mathrm{C}-\mathrm{F},{ }^{3} \mathrm{~J}_{\mathrm{C}-\mathrm{F}}=6.6 \mathrm{~Hz}, \mathrm{C} 5\right), 133.4\left(\mathrm{t}, \mathrm{C}-\mathrm{F},{ }^{2} J_{\mathrm{C}-\mathrm{F}}=27.3 \mathrm{~Hz}\right.$, C4), $132.9\left(\mathrm{t}, \mathrm{C}-\mathrm{F},{ }^{3} \mathrm{~J}_{\mathrm{C}-\mathrm{F}}=6.1 \mathrm{~Hz}, \mathrm{C} 3\right), 123.3(\mathrm{C} 2), 122.3\left(\mathrm{t}, \mathrm{C}-\mathrm{F},{ }^{1} \mathrm{~J}_{\mathrm{C}-\mathrm{F}}=242.4 \mathrm{~Hz}, \mathrm{C} 6\right), 38.9\left(\mathrm{t}, \mathrm{C}-\mathrm{F},{ }^{2} \mathrm{~J}_{\mathrm{C}-\mathrm{F}}=26.8\right.$ $\mathrm{Hz}, \mathrm{C} 7), 24.5$ (t, C-F, $\left.{ }^{3} \mathrm{~J}_{\mathrm{C}-\mathrm{F}}=4.1 \mathrm{~Hz}, \mathrm{C} 8\right), 22.4$ (C9), 11.9 (C10).

${ }^{19} \mathrm{~F}$ NMR (471 MHz, $\left.\mathrm{CDCl}_{3}\right): \delta-95.94\left(\mathrm{t}, \mathrm{F}-\mathrm{H},{ }^{3} \mathrm{~J}_{\mathrm{F}-\mathrm{H}}=21.2 \mathrm{~Hz}, 2 \mathrm{~F}\right)$.

HRMS (ESI): $\mathrm{m} / \mathrm{z}[\mathrm{M}+\mathrm{H}]^{+}$calcd for $\mathrm{C}_{10} \mathrm{H}_{14} \mathrm{~F}_{2} \mathrm{~N}$ : 186.1089; found: 186.1089 .

${ }^{1} \mathrm{H}$ NMR $\left(500 \mathrm{MHz}, \mathrm{CDCl}_{3}\right)$ of $2 \mathrm{f}$ :

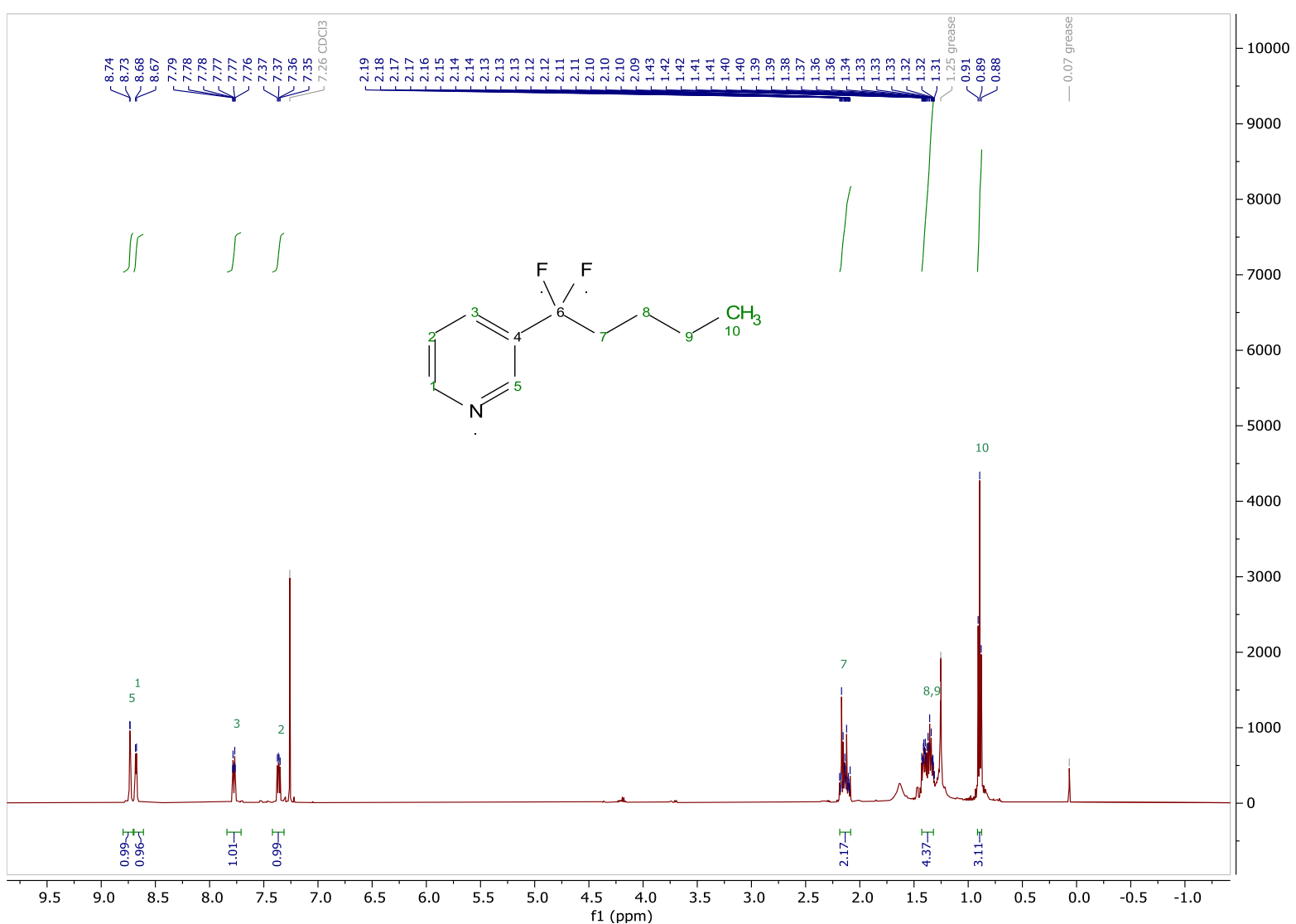


${ }^{13} \mathrm{C}$ NMR $\left(126 \mathrm{MHz}, \mathrm{CDCl}_{3}\right)$ of $2 \mathrm{f}$ :

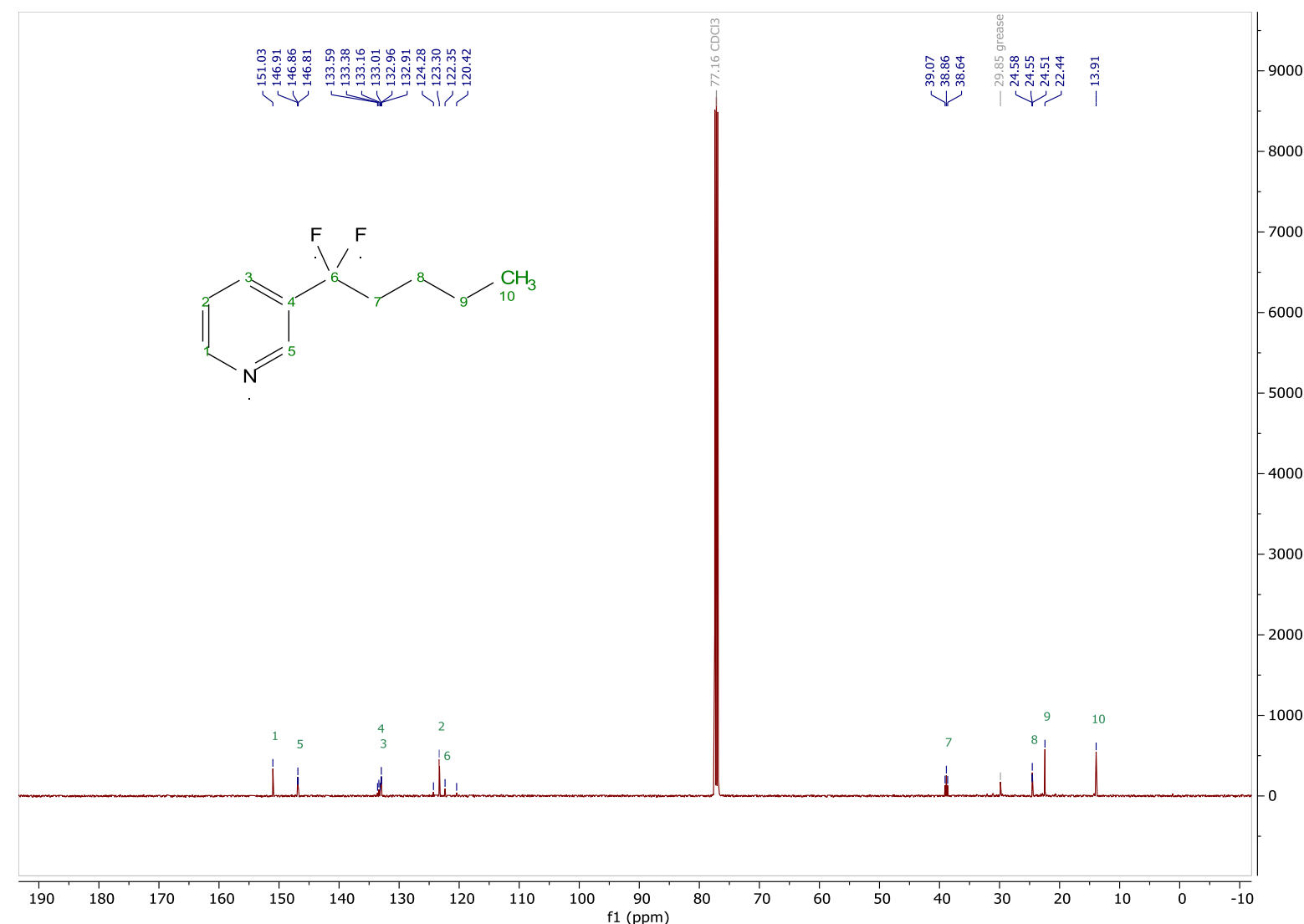

${ }^{19} \mathrm{~F} \mathrm{NMR} \mathrm{(471} \mathrm{MHz}, \mathrm{CDCl}_{3}$ ) of $2 \mathrm{f}$ :

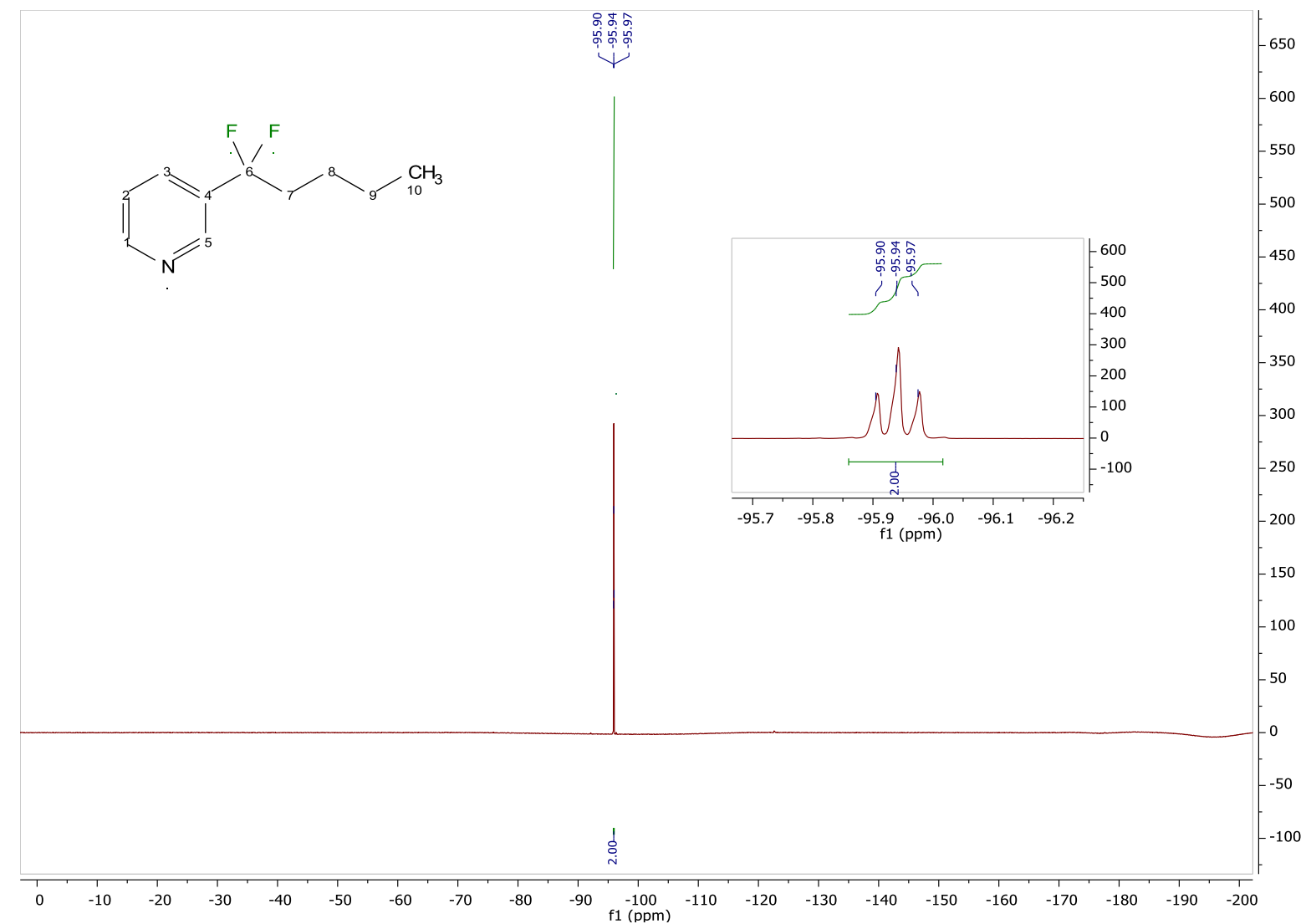


<smiles>[B][Si](C)(C)CC(F)(F)c1cccnc1</smiles>

Sequential quench: $\mathbf{2 g}$ was prepared according to protocol $\mathbf{A}$ using (iodomethyl)trimethylsilane (2.8 equiv., $5.38 \mathrm{mmol}, 0.80 \mathrm{~mL}$ ). $20 \%$ of ${ }^{19} \mathrm{~F}$ NMR yield was calculated with fluorobenzene as internal standard. Purification via flash column chromatography (silica gel, gradient from 0 to $20 \%$ of EtOAc in cyclohexane) yielded $2 \mathrm{~g}$ as a yellow oil ( $62 \mathrm{mg}, 0.29 \mathrm{mmol}, 15 \%)$.

${ }^{1} \mathrm{H}$ NMR (500 MHz, $\left.\mathrm{CDCl}_{3}\right): \delta 8.75-8.74(\mathrm{~m}, 1 \mathrm{H}, \mathrm{H} 5), 8.66-8.61(\mathrm{~m}, 1 \mathrm{H}, \mathrm{H} 1), 7.80-7.74(\mathrm{~m}, 1 \mathrm{H}, \mathrm{H} 3)$, 7.32 (ddd, $J=7.9,4.8,0.8 \mathrm{~Hz}, 1 \mathrm{H}, \mathrm{H} 2$ ), $1.67\left(\mathrm{t}, \mathrm{H}-\mathrm{F},{ }^{3} \mathrm{~J}_{\mathrm{H}-\mathrm{F}}=21.3 \mathrm{~Hz}, 2 \mathrm{H}, \mathrm{H} 7\right), 0.03(\mathrm{~s}, 9 \mathrm{H}, \mathrm{H} 8)$.

${ }^{13} \mathrm{C}$ NMR $\left(126 \mathrm{MHz}, \mathrm{CDCl}_{3}\right): \delta 150.8\left(\mathrm{t}, \mathrm{C}-\mathrm{F},{ }^{5} \mathrm{~J}_{\mathrm{C}-\mathrm{F}}=1.8 \mathrm{~Hz}, \mathrm{C} 1\right), 146.5\left(\mathrm{t}, \mathrm{C}-\mathrm{F},{ }^{3} \mathrm{~J}_{\mathrm{C}-\mathrm{F}}=6.1 \mathrm{~Hz}, \mathrm{C} 5\right), 135.4(\mathrm{t}$, $\left.\mathrm{C}-\mathrm{F},{ }^{2} J_{\mathrm{C}-\mathrm{F}}=28.9 \mathrm{~Hz}, \mathrm{C} 4\right), 132.4\left(\mathrm{t}, \mathrm{C}-\mathrm{F},{ }^{3} J_{\mathrm{C}-\mathrm{F}}=5.8 \mathrm{~Hz}, \mathrm{C} 3\right), 124.5\left(\mathrm{t}, \mathrm{C}-\mathrm{F},{ }^{1} J_{\mathrm{C}-\mathrm{F}}=240.1 \mathrm{~Hz}, \mathrm{C} 6\right), 123.2(\mathrm{C} 2)$, $29.7\left(\mathrm{t}, \mathrm{C}-\mathrm{F},{ }^{2} \mathrm{~J}_{\mathrm{C}-\mathrm{F}}=30.9 \mathrm{~Hz}, \mathrm{C} 7\right),-0.6(\mathrm{C} 8)$.

${ }^{19} \mathrm{~F}$ NMR (471 MHz, $\left.\mathrm{CDCl}_{3}\right): \delta-81.69\left(\mathrm{t}, \mathrm{F}-\mathrm{H},{ }^{3} \mathrm{~J}_{\mathrm{F}-\mathrm{H}}=21.2 \mathrm{~Hz}, 2 \mathrm{~F}\right)$.

${ }^{29} \mathrm{Si}$ NMR $\left(99 \mathrm{MHz}, \mathrm{CDCl}_{3}\right): \delta-0.52\left(\mathrm{t}, \mathrm{Si}-\mathrm{F},{ }^{3} \mathrm{~J}_{\mathrm{Si}-\mathrm{F}}=6.7 \mathrm{~Hz}, 1 \mathrm{Si}\right)$.

HRMS (ESI): $\mathrm{m} / \mathrm{z}[\mathrm{M}+\mathrm{H}]^{+}$calcd for $\mathrm{C}_{10} \mathrm{H}_{16} \mathrm{~F}_{2} \mathrm{NSi}$ : 216.1015; found: 216.1007 .

${ }^{1} \mathrm{H}$ NMR $\left(500 \mathrm{MHz}, \mathrm{CDCl}_{3}\right)$ of $2 \mathrm{~g}$ :

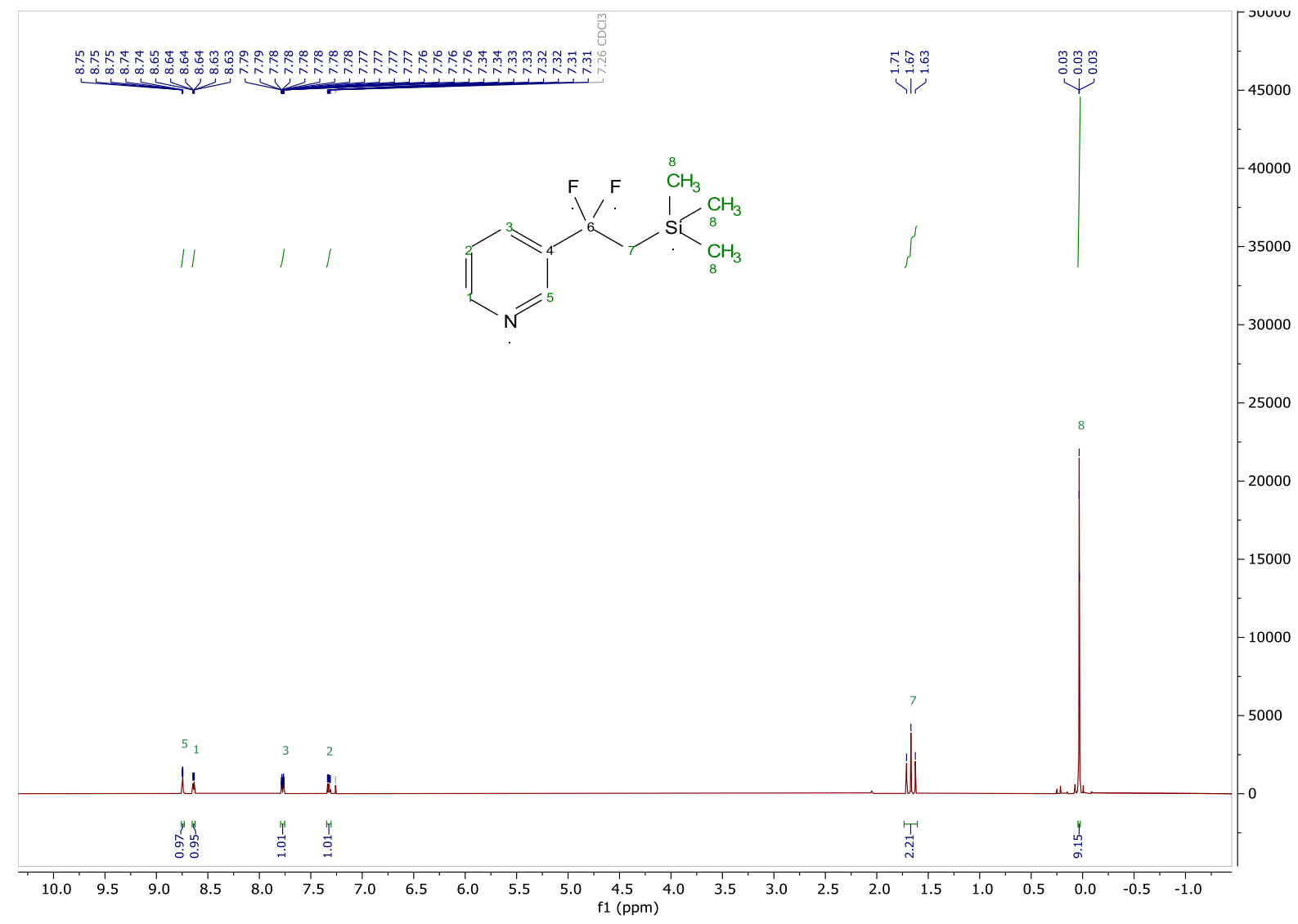


${ }^{13} \mathrm{C}$ NMR $\left(126 \mathrm{MHz}, \mathrm{CDCl}_{3}\right)$ of $2 \mathrm{~g}$ :

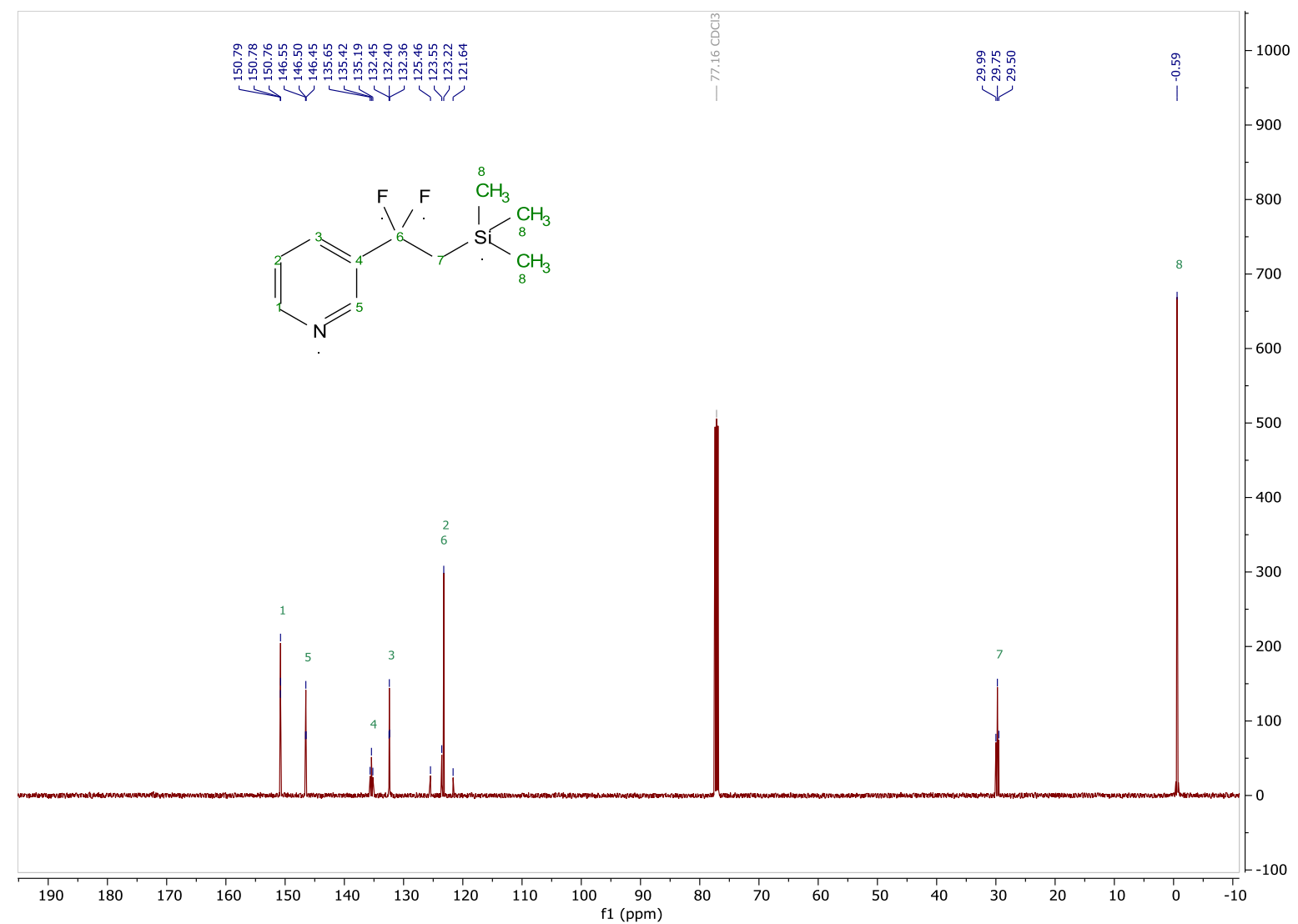

$\left.{ }^{29} \mathrm{Si} \mathrm{NMR} \mathrm{(471} \mathrm{MHz,} \mathrm{CDCl}_{3}\right)$ of $2 \mathrm{~g}$ :

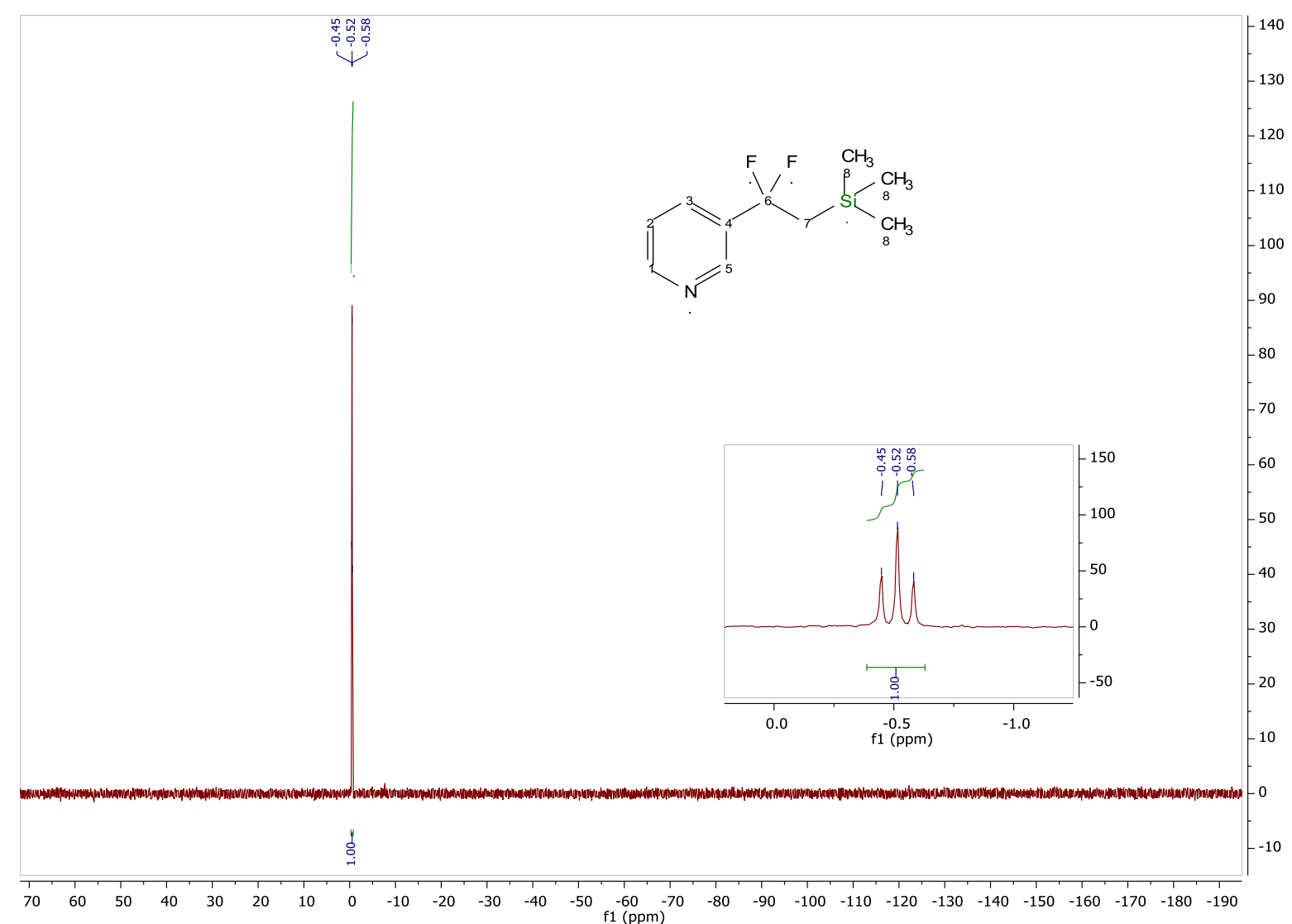




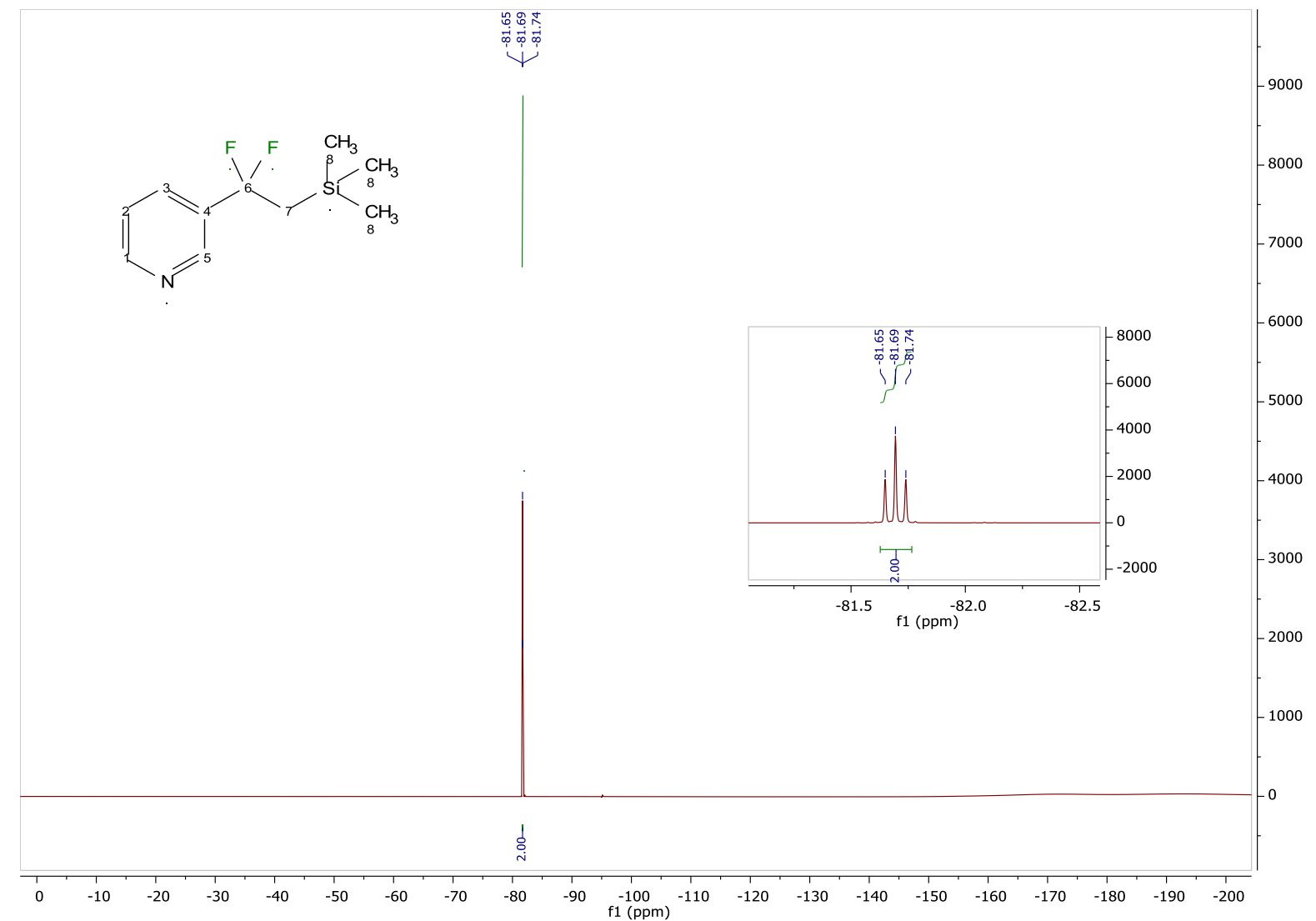

Ethyl 2,2-difluoro-2-(pyridin-3-yl)acetate (2h)<smiles>CCOC(=O)C(F)(F)c1cccnc1</smiles>

\section{By sequential quench:}

$2 \mathrm{~h}$ was prepared according to protocol A using ethyl chloroformate (5 equiv., $9.62 \mathrm{mmol}, 0.92 \mathrm{~mL}$ ). $29 \%$ of ${ }^{19} \mathrm{~F}$ NMR yield was calculated with fluorobenzene as internal standard. Purification via flash column chromatography (silica gel, gradient from 0 to $20 \%$ of EtOAc in cyclohexane) yielded $\mathbf{2 h}$ as a colorless gum (67 mg, $0.12 \mathrm{mmol}, 16 \%$ ).

\section{By desilylative post-functionalization:}

Ethyl chloroformate (1.2 equiv., $0.21 \mathrm{mmol}, 0.02 \mathrm{~mL}$ ) was added to a solution of TBAT (1 equiv., 0.17 mmol, $90 \mathrm{mg})$ in DMF $(1 \mathrm{~mL})$ at $0{ }^{\circ} \mathrm{C}$ and under inert atmosphere. A solution of 3((dimethyl(phenyl)silyl)difluoromethyl)pyridine $\mathbf{2 b}$ (1 equiv., $0.17 \mathrm{mmol}, 44 \mathrm{mg}$ ) in DMF $(0.5 \mathrm{~mL}$ ) precooled to $0{ }^{\circ} \mathrm{C}$ was added to the reaction mixture, which was stirred for $17 \mathrm{~h}$ at $100{ }^{\circ} \mathrm{C}$. $26 \%$ of ${ }^{19} \mathrm{~F} \mathrm{NMR}$ yield was calculated with fluorobenzene as internal standard. Purification via flash column chromatography (silica gel, gradient from 0 to $20 \%$ of EtOAc in cyclohexane) yielded $\mathbf{2} \mathbf{h}$ as a colorless gum (7 mg, $0.03 \mathrm{mmol}, 21 \%)$.

${ }^{1} \mathrm{H}$ NMR (500 MHz, CDCl 3 ): $\delta 8.86(\mathrm{~d}, J=1.3 \mathrm{~Hz}, 1 \mathrm{H}, \mathrm{H} 5), 8.74(\mathrm{~d}, J=4.9 \mathrm{~Hz}, 1 \mathrm{H}, \mathrm{H} 1), 7.99-7.88(\mathrm{~m}, 1 \mathrm{H}$, $\mathrm{H} 3$ ), 7.40 (ddd, $J=7.9,4.9,0.8 \mathrm{~Hz}, 1 \mathrm{H}, \mathrm{H} 2$ ), 4.31 (q, $J=7.1 \mathrm{~Hz}, 2 \mathrm{H}, \mathrm{H} 8), 1.31$ (t, $J=7.1 \mathrm{~Hz}, 3 \mathrm{H}, \mathrm{H} 9$ ). 
${ }^{13} \mathrm{C}$ NMR (126 MHz, CDCl $): \delta 163.5\left(\mathrm{t}, \mathrm{C}-\mathrm{F},{ }^{2} \mathrm{~J}_{\mathrm{C}-\mathrm{F}}=34.7 \mathrm{~Hz}, \mathrm{C} 7\right), 152.3(\mathrm{C} 1), 147.2\left(\mathrm{t}, \mathrm{C}-\mathrm{F},{ }^{3} \mathrm{~J}_{\mathrm{C}-\mathrm{F}}=6.5 \mathrm{~Hz}\right.$, C5), $133.6\left(\mathrm{t}, \mathrm{C}-\mathrm{F},{ }^{3} \mathrm{~J}_{\mathrm{C}-\mathrm{F}}=5.9 \mathrm{~Hz}, \mathrm{C} 3\right), 129.0\left(\mathrm{t}, \mathrm{C}-\mathrm{F},{ }^{2} \mathrm{~J}_{\mathrm{C}-\mathrm{F}}=26.0 \mathrm{~Hz}, \mathrm{C} 4\right), 123.4(\mathrm{C} 2), 112.6\left(\mathrm{t}, \mathrm{C}-\mathrm{F},{ }^{1} \mathrm{~J}_{\mathrm{C}-\mathrm{F}}=\right.$ $253.1 \mathrm{~Hz}, \mathrm{C} 6), 63.7$ (C8), 13.9 (C9).

${ }^{19} \mathrm{~F}$ NMR (471 MHz, $\left.\mathrm{CDCl}_{3}\right): \delta-104.50(2 \mathrm{~F})$.

HRMS (ESI): $\mathrm{m} / \mathrm{z}[\mathrm{M}+\mathrm{H}]^{+}$calcd for $\mathrm{C}_{9} \mathrm{H}_{10} \mathrm{~F}_{2} \mathrm{NO}_{2}: 202.0674$; found: 202.0660 .

These data are in accordance with previously reported results. ${ }^{5}$

${ }^{1} \mathrm{H}$ NMR $\left(500 \mathrm{MHz}, \mathrm{CDCl}_{3}\right.$ ) of $2 \mathrm{~h}$ :

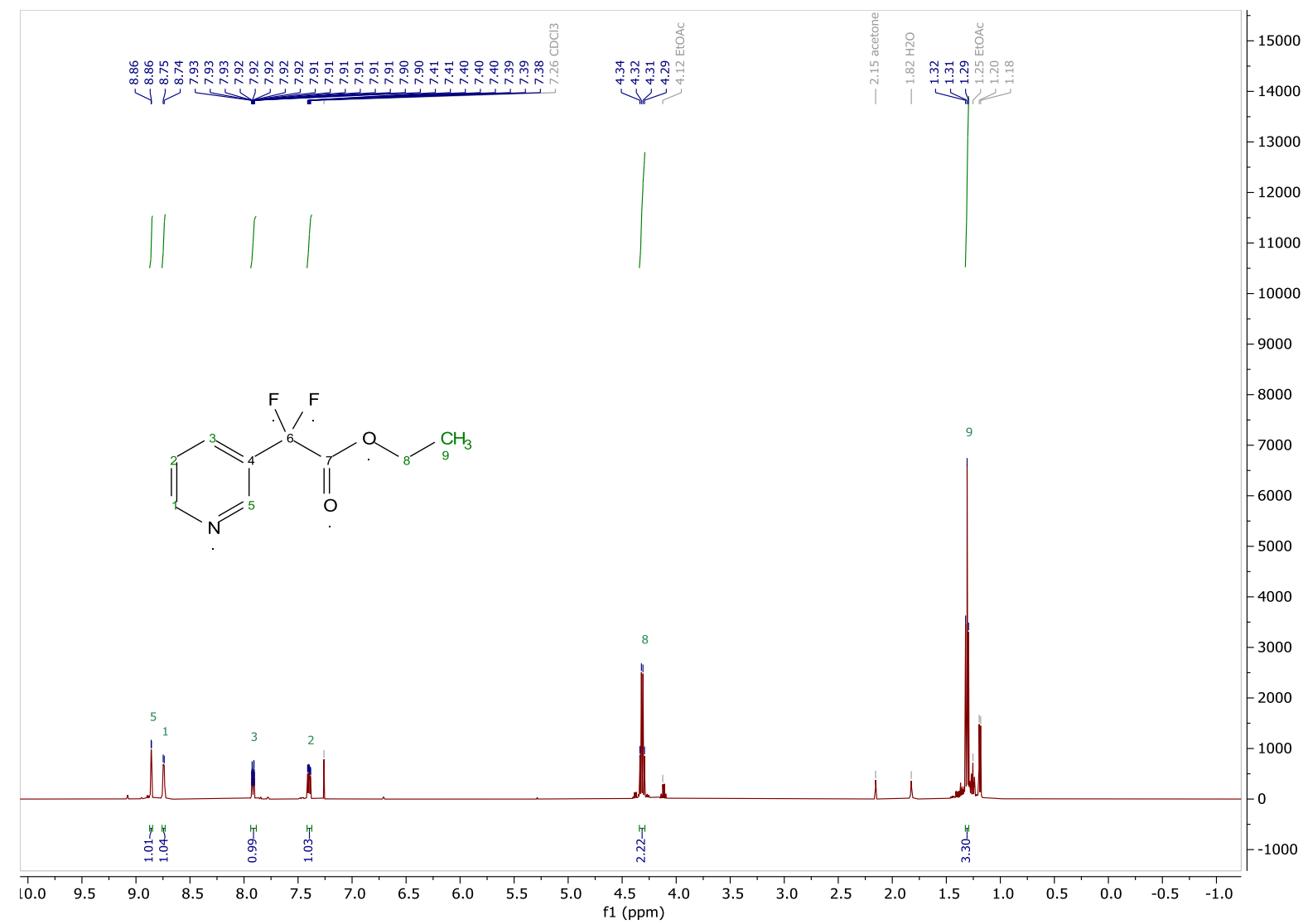


${ }^{13} \mathrm{C}$ NMR $\left(126 \mathrm{MHz}, \mathrm{CDCl}_{3}\right)$ of $2 \mathrm{~h}$ :

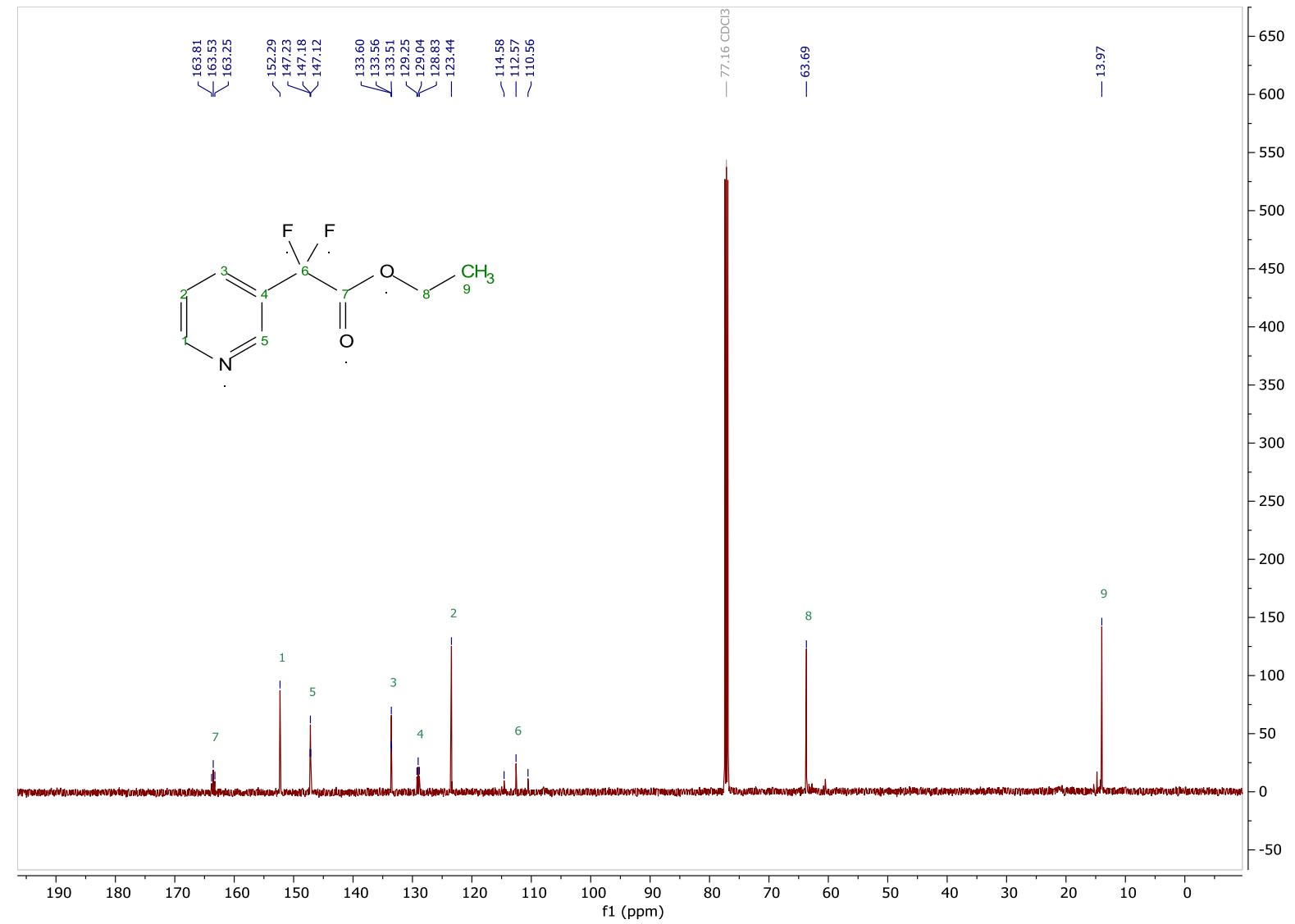

${ }^{19} \mathrm{~F} \mathrm{NMR}\left(471 \mathrm{MHz}, \mathrm{CDCl}_{3}\right)$ of $2 \mathrm{~h}$ :

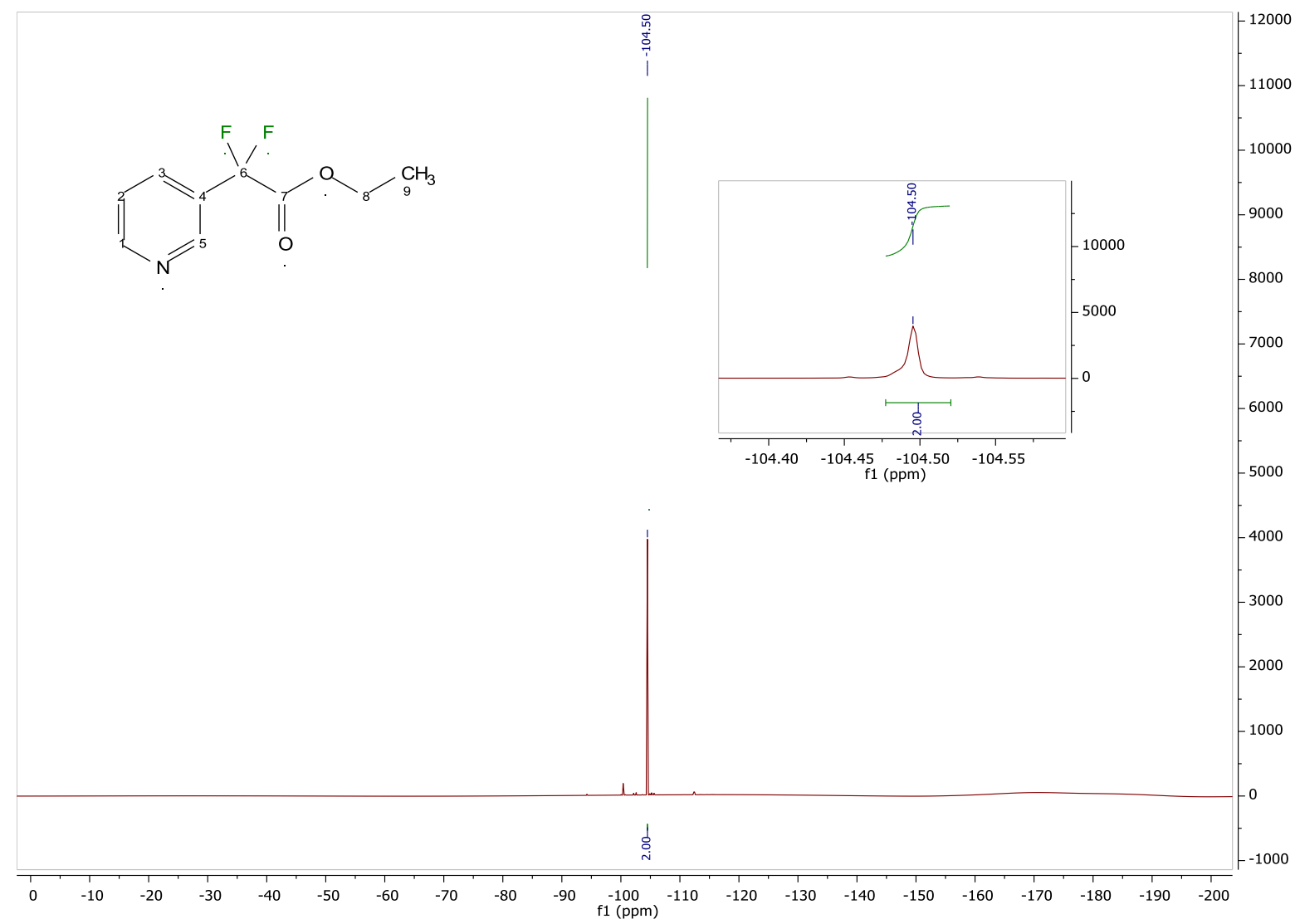


<smiles>FC(F)(Sc1ccccc1)c1cccnc1</smiles>

\section{By sequential quench:}

2i was prepared according to protocol A using diphenyl disulfide (2 equiv., $3.85 \mathrm{mmol}, 840 \mathrm{mg}$ ). 15\% of ${ }^{19} \mathrm{~F}$ NMR yield was calculated with fluorobenzene as internal standard. Purification via flash column chromatography (silica gel, gradient from 0 to $15 \%$ of EtOAc in cyclohexane) yielded $\mathbf{2} \mathbf{i}$ as a yellow amorphous solid (36 mg, $0.15 \mathrm{mmol}, 8 \%$ ).

\section{By desilylative post-functionalization:}

Diphenyl disulfide (1 equiv., $0.49 \mathrm{mmol}, 107 \mathrm{mg}$ ) was added to a solution of 3((dimethyl(phenyl)silyl)difluoromethyl)pyridine $\mathbf{2 b}$ (1 equiv., $0.49 \mathrm{mmol}, 128 \mathrm{mg}$ ) in DMF $(1 \mathrm{~mL})$ at $0{ }^{\circ} \mathrm{C}$ and under inert atmosphere. A solution of tetrabutylammonium difluorotriphenylsilicate (TBAT) (1 equiv., $0.49 \mathrm{mmol}, 264 \mathrm{mg})$ in DMF $(3 \mathrm{~mL})$ pre-cooled to $0{ }^{\circ} \mathrm{C}$ was added to the reaction mixture, which was then stirred for $18 \mathrm{~h}$ at $80{ }^{\circ} \mathrm{C} .65 \%$ of ${ }^{19} \mathrm{~F}$ NMR yield was calculated with fluorobenzene as internal standard. Purification via flash column chromatography (silica gel, gradient from 0 to $15 \%$ of EtOAc in cyclohexane) yielded $\mathbf{2} \mathbf{i}$ as a yellow amorphous solid ( $66 \mathrm{mg}, 0.28 \mathrm{mmol}, 57 \%$ ).

${ }^{1} \mathrm{H}$ NMR (500 MHz, $\left.\mathrm{CDCl}_{3}\right): \delta 8.80-8.76(\mathrm{~m}, 1 \mathrm{H}, \mathrm{H} 5), 8.68(\mathrm{~d}, J=4.4 \mathrm{~Hz}, 1 \mathrm{H}, \mathrm{H} 1), 7.86-7.78(\mathrm{~m}, 1 \mathrm{H}$, $\mathrm{H} 3), 7.61-7.55(\mathrm{~m}, 2 \mathrm{H}, \mathrm{H} 8), 7.47-7.41(\mathrm{~m}, 1 \mathrm{H}, \mathrm{H} 10), 7.40-7.30(\mathrm{~m}, 3 \mathrm{H}, \mathrm{H} 2, \mathrm{H} 9)$.

${ }^{13} \mathrm{C}$ NMR $\left(126 \mathrm{MHz}, \mathrm{CDCl}_{3}\right): \delta 151.8\left(\mathrm{t}, \mathrm{C}-\mathrm{F},{ }^{5} \mathrm{~J}_{\mathrm{C}-\mathrm{F}}=1.8 \mathrm{~Hz}, \mathrm{C} 1\right), 147.0\left(\mathrm{t}, \mathrm{C}-\mathrm{F},{ }^{3} J_{\mathrm{C}-\mathrm{F}}=4.9 \mathrm{~Hz}, \mathrm{C} 5\right), 136.6$ (C8), $133.2\left(\mathrm{t}, \mathrm{C}-\mathrm{F},{ }^{3} \mathrm{~J}_{\mathrm{C}-\mathrm{F}}=4.5 \mathrm{~Hz}, \mathrm{C} 3\right), 132.1\left(\mathrm{t}, \mathrm{C}-\mathrm{F},{ }^{2} \mathrm{~J}_{\mathrm{C}-\mathrm{F}}=25.9 \mathrm{~Hz}, \mathrm{C} 4\right), 130.4$ (C10), 129.3 (C9), 126.8 (t, C-F, $\left.{ }^{1} J_{C-F}=279.2 \mathrm{~Hz}, C 6\right), 126.8\left(\mathrm{t}, \mathrm{C}-\mathrm{F},{ }^{3} \mathrm{~J}_{\mathrm{C}-\mathrm{F}}=1.8 \mathrm{~Hz}, \mathrm{C} 7\right), 123.1(\mathrm{C} 2)$.

${ }^{19} \mathrm{~F}$ NMR (471 MHz, $\left.\mathrm{CDCl}_{3}\right): \delta-72.65(2 \mathrm{~F})$.

HRMS (ESI): $\mathrm{m} / \mathrm{z}[\mathrm{M}+\mathrm{H}]^{+}$calcd for $\mathrm{C}_{12} \mathrm{H}_{10} \mathrm{~F}_{2} \mathrm{NS}: 238.0497$; found: 238.0478 .

FTIR (cm ${ }^{-1}$, neat): 3060, 1591, 1580, 1475, 1441, 1422, 1268, 1057, 1033, 1017, 963, 933, 910, 803, 748, 719, 702, 631, 611, 500.

These data are in accordance with previously reported results. ${ }^{6}$ 
${ }^{1} \mathrm{H}$ NMR (500 MHz, $\mathrm{CDCl}_{3}$ ) of 2i:

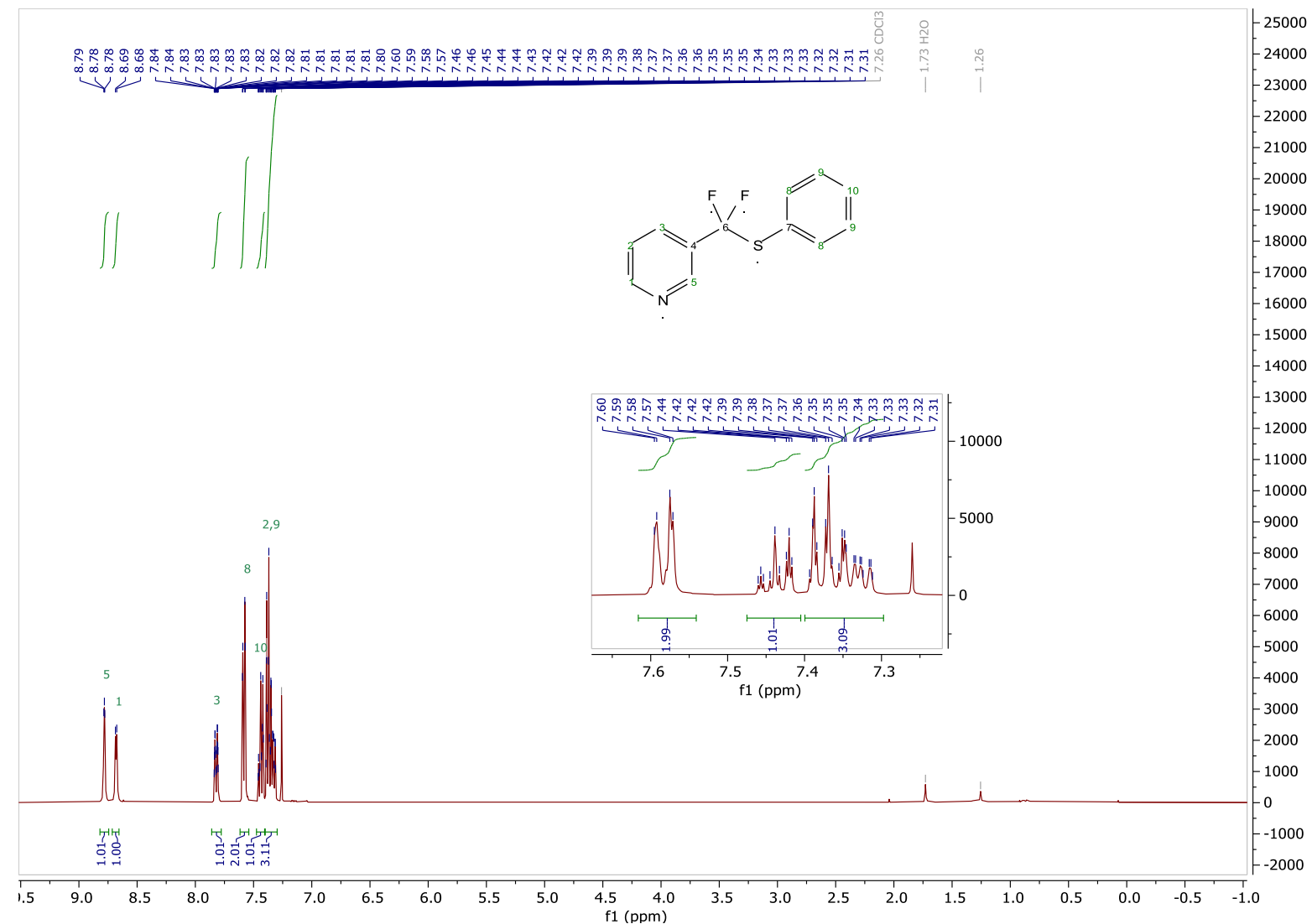

${ }^{13} \mathrm{C}$ NMR $\left(126 \mathrm{MHz} \mathrm{CDCl}_{3}\right)$ of 2i:

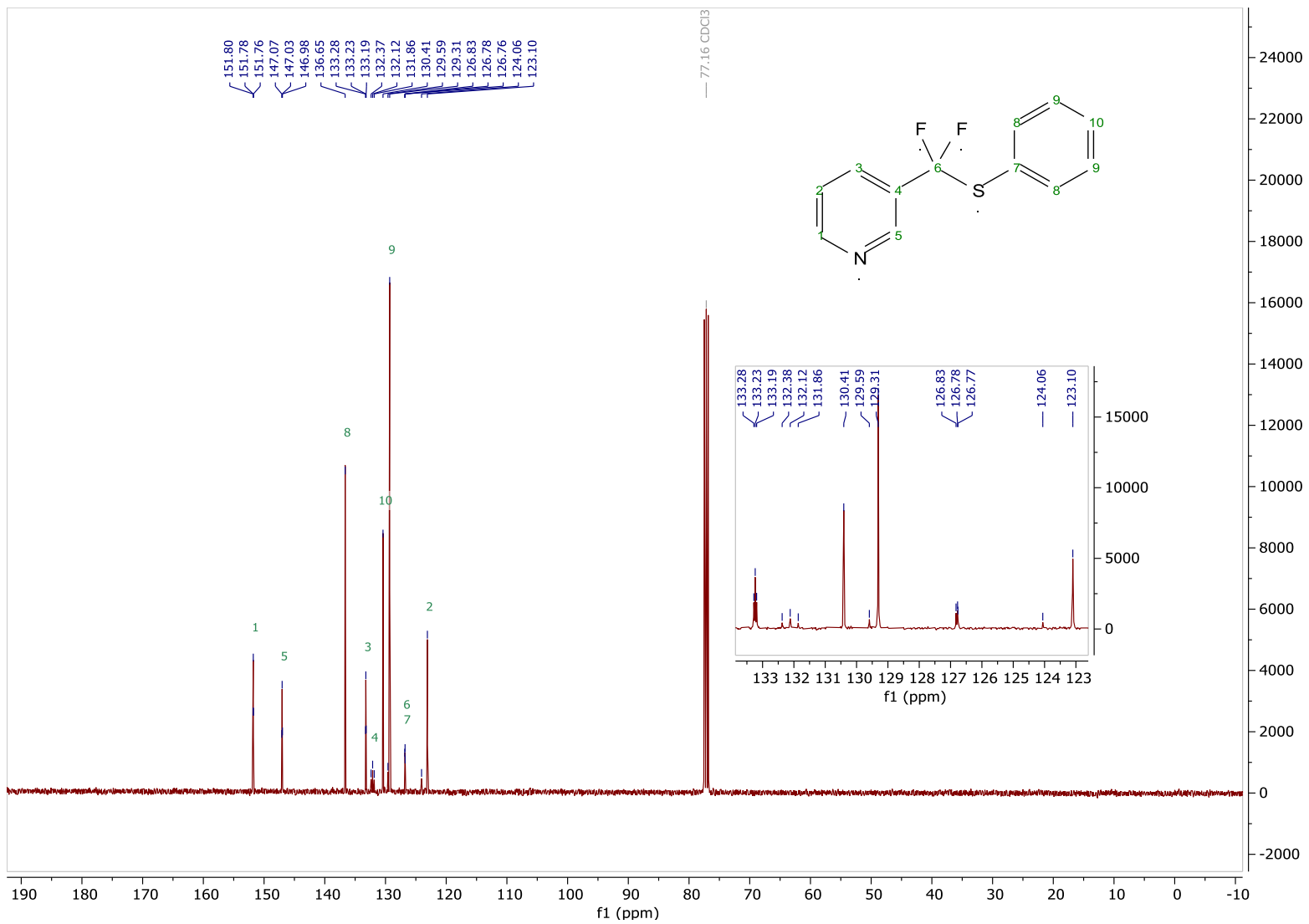




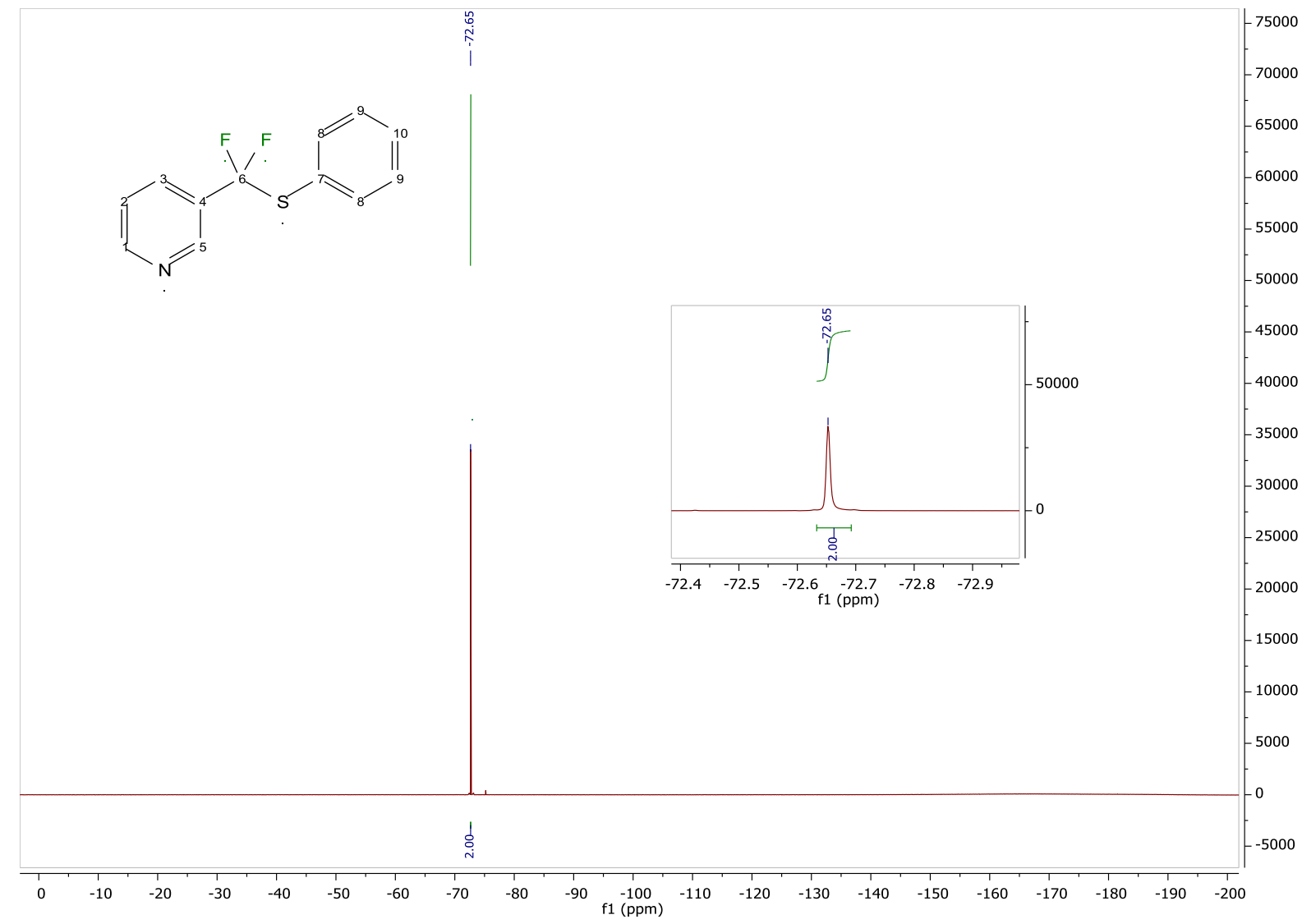

3-(Difluoro(tributylstannyl)methyl)pyridine (2j)<smiles>CCCCCCCCC(C)C(F)(F)c1cccnc1</smiles>

Sequential quench: $\mathbf{2} \mathbf{j}$ was prepared according to protocol $\mathbf{A}$ using tributyltin chloride (2 equiv., 3.85 mmol, $1.1 \mathrm{~mL}$ ). $72 \%$ of ${ }^{19} \mathrm{~F} \mathrm{NMR}$ yield was calculated with fluorobenzene as internal standard. Purification via flash column chromatography (silica gel, gradient from 0 to $15 \%$ of EtOAc in cyclohexane) yielded $\mathbf{2} \mathbf{j}$ as a yellow amorphous solid (552 $\mathrm{mg}, 1.32 \mathrm{mmol}, 69 \%$ ).

${ }^{1} \mathrm{H}$ NMR (400 MHz, $\left.\mathrm{CDCl}_{3}\right): \delta 8.57(\mathrm{~s}, 2 \mathrm{H}, \mathrm{H} 1, \mathrm{H} 5), 7.65-7.52(\mathrm{~m}, 1 \mathrm{H}, \mathrm{H} 3), 7.31$ (dd, J = 8.1, 4.8 Hz, $1 \mathrm{H}$, $\mathrm{H} 2), 1.51-1.40(\mathrm{~m}, 6 \mathrm{H}, \mathrm{H} 8), 1.31-1.20(\mathrm{~m}, 6 \mathrm{H}, \mathrm{H} 9), 1.05-0.98(\mathrm{~m}, 6 \mathrm{H}, \mathrm{H} 7), 0.86(\mathrm{t}, J=7.3 \mathrm{~Hz}, 9 \mathrm{H}$, $\mathrm{H} 10)$.

${ }^{13} \mathrm{C}$ NMR $\left(101 \mathrm{MHz}, \mathrm{CDCl}_{3}\right): \delta 149.6\left(\mathrm{t}, \mathrm{C}-\mathrm{F},{ }^{5} \mathrm{~J}_{\mathrm{C}-\mathrm{F}}=2.9 \mathrm{~Hz}, \mathrm{C} 1\right), 145.5\left(\mathrm{t}, \mathrm{C}-\mathrm{F},{ }^{3} \mathrm{~J}_{\mathrm{C}-\mathrm{F}}=8.9 \mathrm{~Hz}, \mathrm{C} 5\right), 137.5(\mathrm{t}$, $\left.\mathrm{C}-\mathrm{F},{ }^{2} J_{\mathrm{C}-\mathrm{F}}=17.4 \mathrm{~Hz}, \mathrm{C} 4\right), 132.8\left(\mathrm{t}, \mathrm{C}-\mathrm{F},{ }^{1} J_{\mathrm{C}-\mathrm{F}}=293.4 \mathrm{~Hz}, \mathrm{C} 6\right), 131.0\left(\mathrm{t}, \mathrm{C}-\mathrm{F},{ }^{3} J_{\mathrm{C}-\mathrm{F}}=8.1 \mathrm{~Hz}, \mathrm{C} 3\right), 123.3(\mathrm{C} 2)$, 28.6 (C8), 27.3 (C9), 13.7 (C10), 10.2 (C7).

${ }^{19} \mathrm{~F}$ NMR (376 MHz, $\left.\mathrm{CDCl}_{3}\right): \delta-98.41(2 \mathrm{~F})$.

HRMS (ESI): $\mathrm{m} / \mathrm{z}[\mathrm{M}+\mathrm{H}]^{+}$calcd for $\mathrm{C}_{18} \mathrm{H}_{32} \mathrm{~F}_{2} \mathrm{NSn}: 420.1519$; found: 420.1523 .

FTIR (cm ${ }^{-1}$, neat): 2957, 2924, 2854, 1590, 1576, 1464, 1417, 1248, 1050, 1031, 1019, 988, 799, 714, 695. 
${ }^{1} \mathrm{H}$ NMR (400 MHz, $\mathrm{CDCl}_{3}$ ) of 2j:

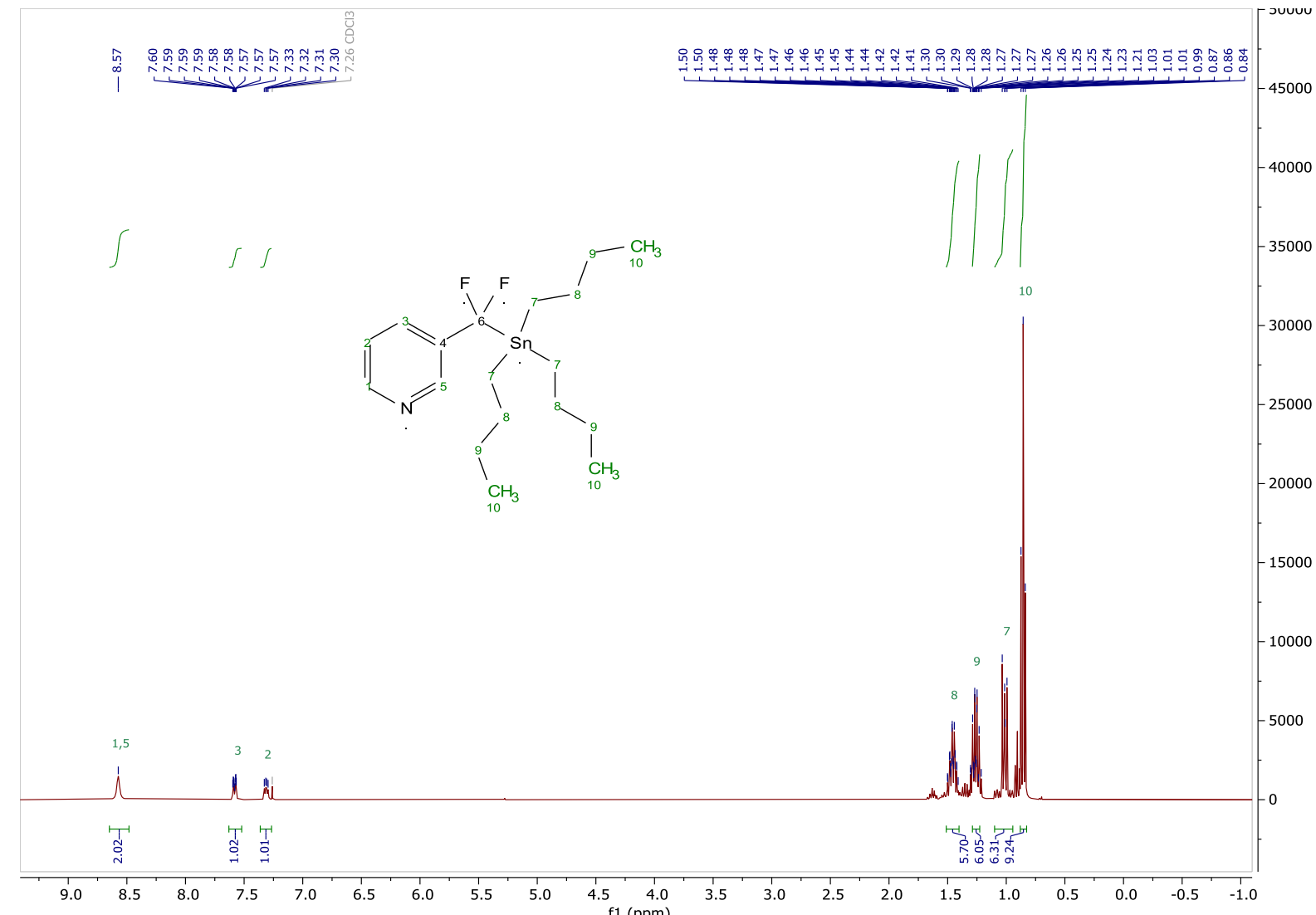

${ }^{13} \mathrm{C}$ NMR (101 MHz, $\mathrm{CDCl}_{3}$ ) of 2j:

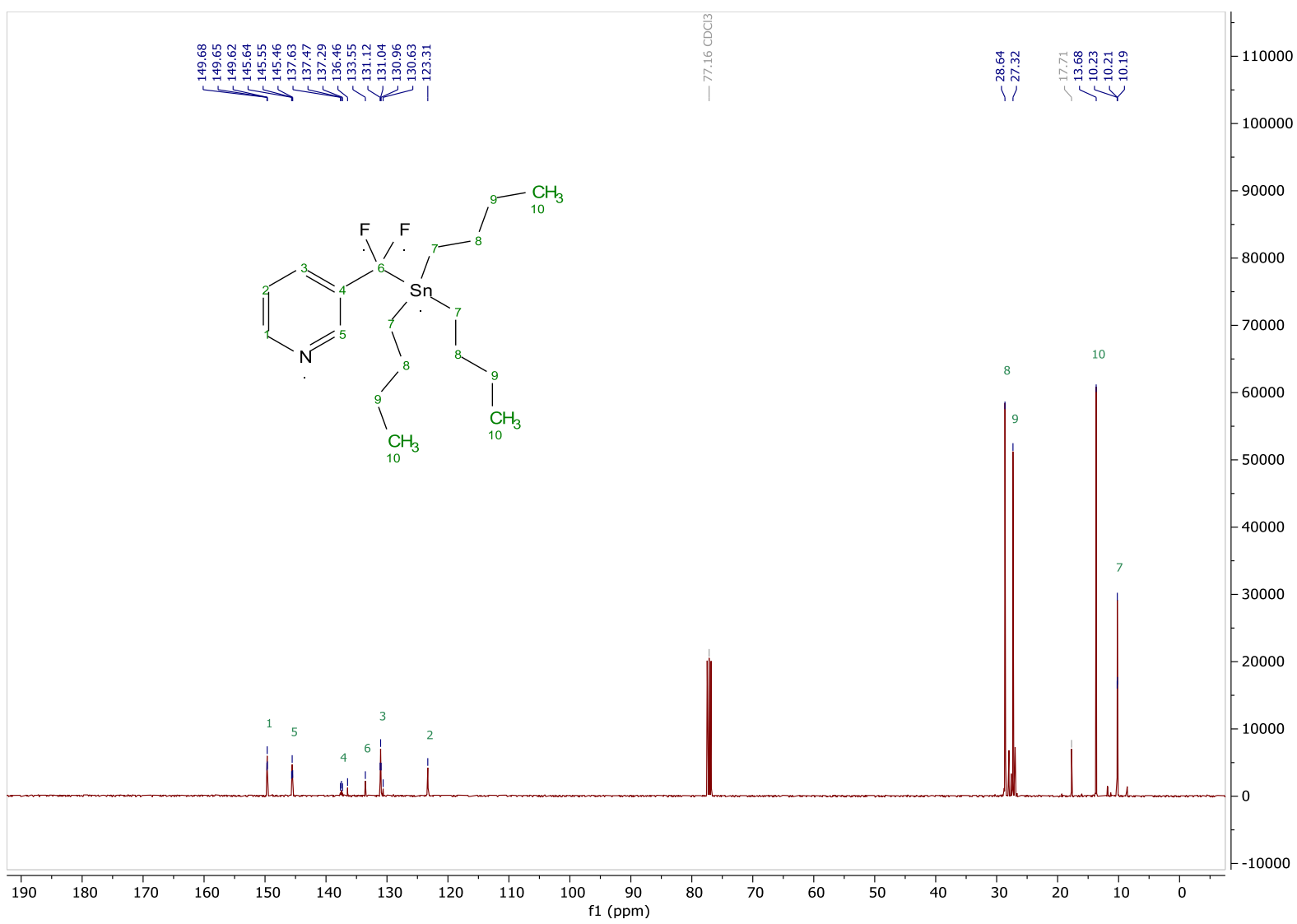




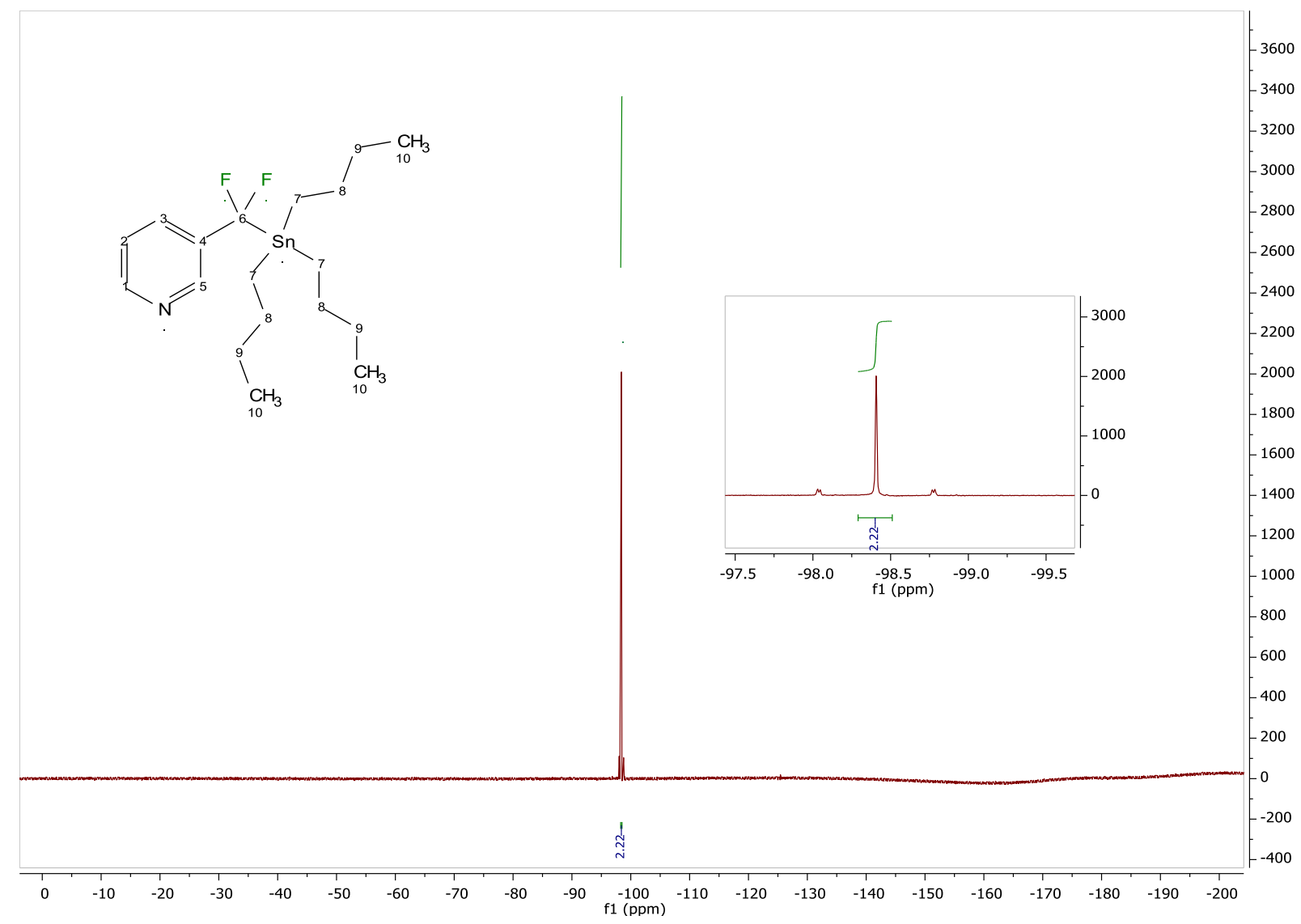

\section{3-(difluoro(phenylselanyl)methyl)pyridine (2k)}<smiles>FC(F)([Se]c1ccccc1)c1cccnc1</smiles>

\section{By sequential quench:}

$\mathbf{2 k}$ was prepared according to protocol A using phenylselenyl chloride (1.3 equiv., $2.56 \mathrm{mmol}, 491 \mathrm{mg}$ ). $4 \%$ of ${ }^{19} \mathrm{~F}$ NMR yield was calculated with fluorobenzene as internal standard. Purification via flash column chromatography (silica gel, gradient from 0 to $20 \%$ of EtOAc in cyclohexane) yielded $\mathbf{2 k}$ as a yellow oil (11 mg, $0.04 \mathrm{mmol}, 2 \%$ ).

\section{By desilylative post-functionalization:}

Phenylselenyl chloride (1 equiv., $0.14 \mathrm{mmol}, 27.6 \mathrm{mg}$ ) was added to a solution of 3((dimethyl(phenyl)silyl)difluoromethyl)pyridine $\mathbf{2 b}$ (1 equiv., $0.14 \mathrm{mmol}, 38 \mathrm{mg}$ ) in DMF $(0.4 \mathrm{~mL}$ ) at 0 ${ }^{\circ} \mathrm{C}$ and under inert atmosphere. A solution of tetrabutylammonium difluorotriphenylsilicate (TBAT) (1 equiv., $0.15 \mathrm{mmol}, 80.9 \mathrm{mg})$ in DMF $(0.7 \mathrm{~mL})$ pre-cooled to $0{ }^{\circ} \mathrm{C}$ was added to the reaction mixture, which was then stirred for $14 \mathrm{~h}$ at $80{ }^{\circ} \mathrm{C} .55 \%$ of ${ }^{19} \mathrm{~F} \mathrm{NMR}$ yield was calculated with fluorobenzene as internal standard. Purification via flash column chromatography (silica gel, gradient from 0 to $20 \%$ of EtOAc in cyclohexane) yielded $\mathbf{2 k}$ as a yellow oil (39 $\mathrm{mg}, 0.056 \mathrm{mmol}, 39 \%)$.

${ }^{1} \mathrm{H}$ NMR $\left(500 \mathrm{MHz}, \mathrm{CDCl}_{3}\right)$ : $\delta 8.67(\mathrm{~s}, 1 \mathrm{H}, \mathrm{H} 5), 8.63(\mathrm{~d}, J=4.7 \mathrm{~Hz}, 1 \mathrm{H}, \mathrm{H} 1), 7.76-7.67(\mathrm{~m}, 1 \mathrm{H}, \mathrm{H} 3), 7.62$ $-7.55(\mathrm{~m}, 2 \mathrm{H}, \mathrm{H} 8), 7.46-7.37$ (m, 1H, H10), $7.34-7.29$ (m, 2H, H9), $7.29-7.26(\mathrm{~m}, 1 \mathrm{H}, \mathrm{H} 2)$. 
${ }^{13} \mathrm{C}$ NMR (126 MHz, CDCl 3 ): $\delta 151.5$ (C1), $146.5\left(\mathrm{t}, \mathrm{C}-\mathrm{F},{ }^{3} \mathrm{~J}_{\mathrm{C}-\mathrm{F}}=5.4 \mathrm{~Hz}, \mathrm{C} 5\right), 137.4$ (C8), 133.2 (C4), 132.7 (t, C-F, $\left.{ }^{3} J_{C-F}=4.9 \mathrm{~Hz}, C 3\right), 130.0$ (C10), $129.4(C 9), 125.1\left(\mathrm{t}, \mathrm{C}-\mathrm{F},{ }^{4} \mathrm{~J}_{\mathrm{C}-\mathrm{F}}=1.4 \mathrm{~Hz}, \mathrm{C} 2\right), 123.2\left(\mathrm{t}, \mathrm{C}-\mathrm{F},{ }^{1} \mathrm{~J}_{\mathrm{C}-\mathrm{F}}=\right.$ $292.9 \mathrm{~Hz}, \mathrm{C} 6), 123.0$ (C7).

${ }^{19}$ F NMR (471 MHz, $\left.\mathrm{CDCl}_{3}\right): \delta-71.31(2 \mathrm{~F})$.

HRMS (ESI): $\mathrm{m} / \mathrm{z}[\mathrm{M}+\mathrm{H}]^{+}$calcd for $\mathrm{C}_{12} \mathrm{H}_{10} \mathrm{~F}_{2} \mathrm{NSe}$ : 285.9941; found: 285.9956.

FTIR (cm ${ }^{-1}$, neat): 1731, 1694, 1590, 1579, 1477, 1440, 1422, 1268, 1057, 1017, 893, 802, 741, 715, 690.

${ }^{1} \mathrm{H}$ NMR $\left(500 \mathrm{MHz}, \mathrm{CDCl}_{3}\right)$ of $2 \mathrm{k}$ :

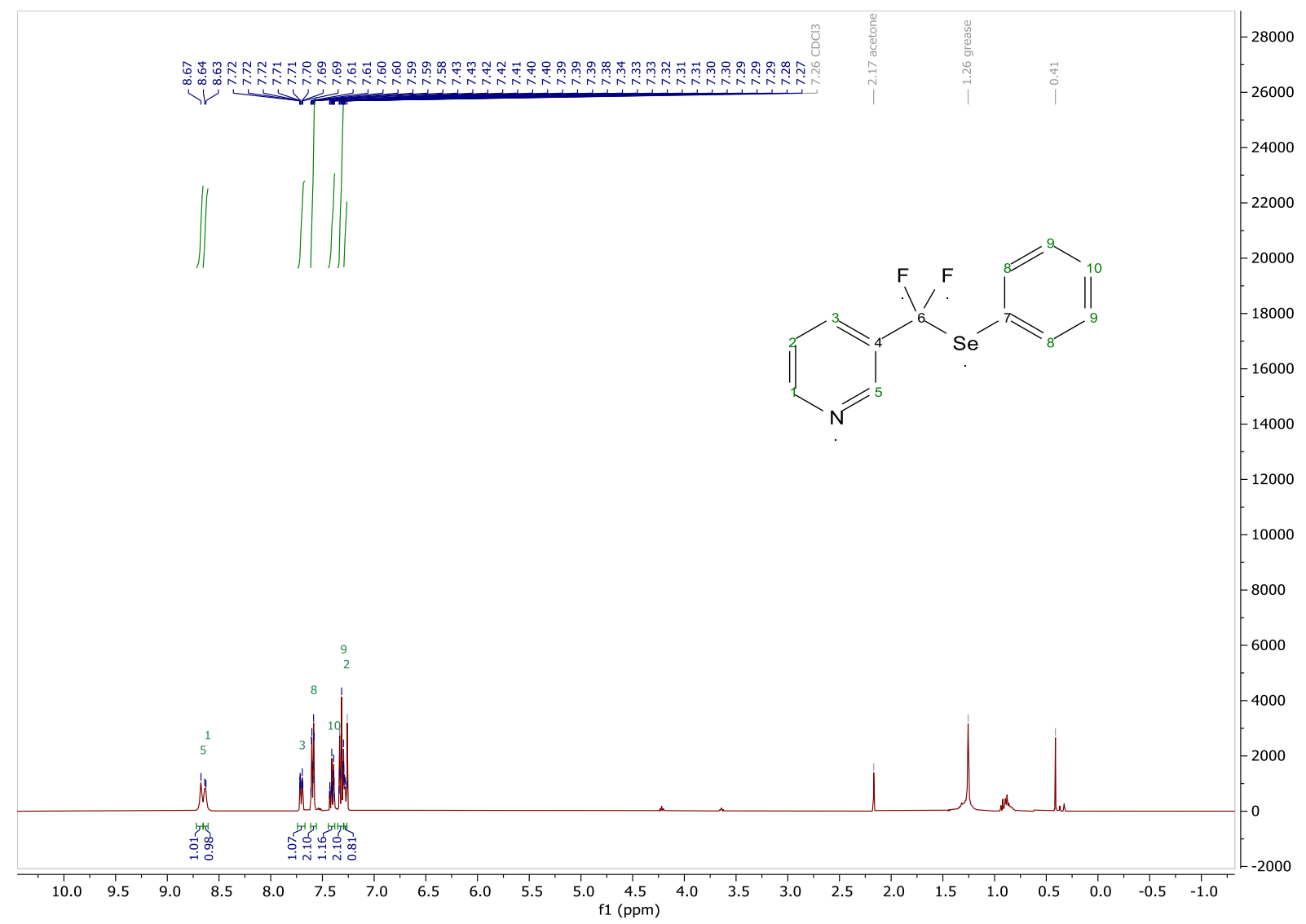


${ }^{13} \mathrm{C}$ NMR $\left(126 \mathrm{MHz}, \mathrm{CDCl}_{3}\right)$ of $2 \mathrm{k}$ :

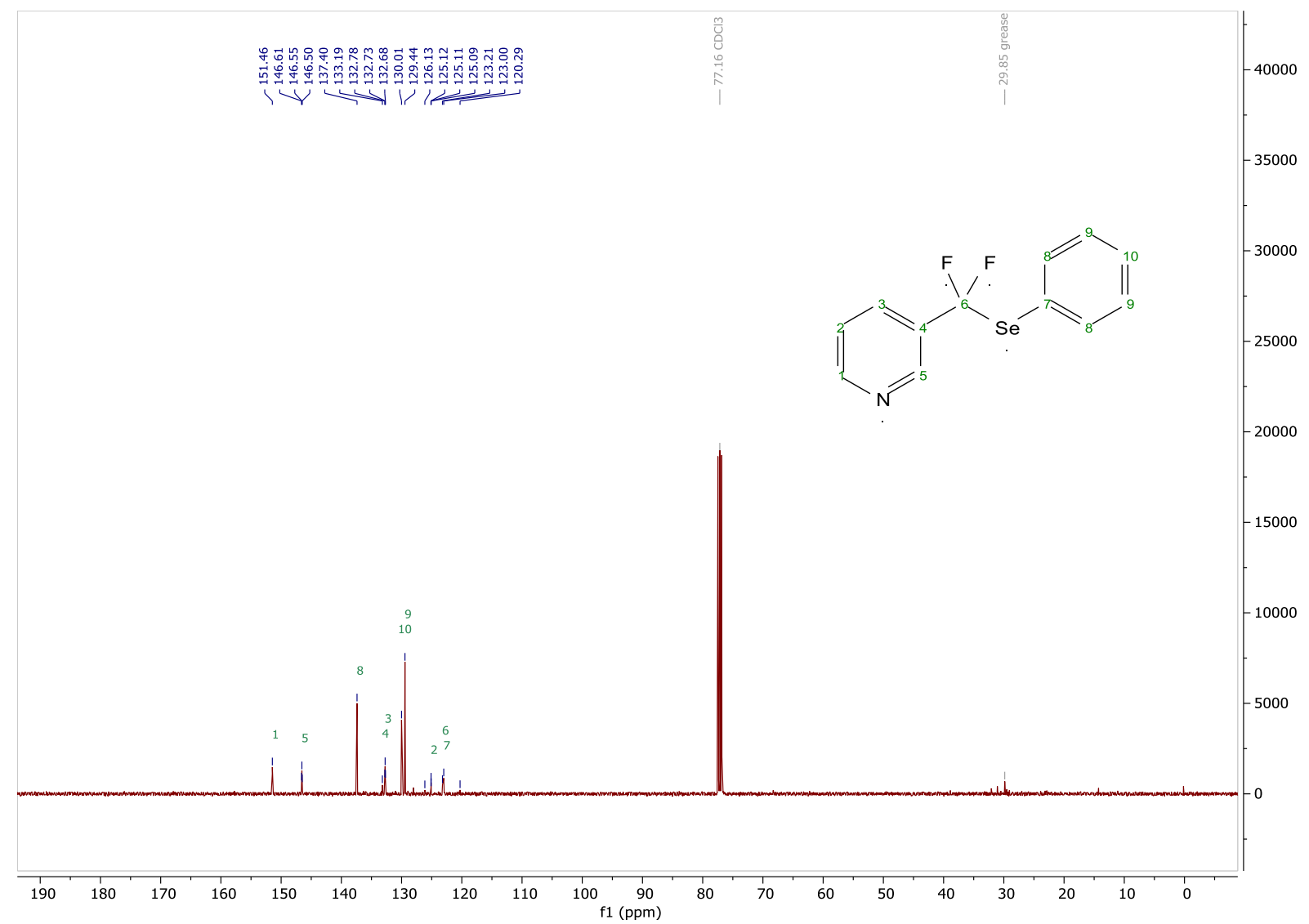

${ }^{19} \mathrm{~F} \mathrm{NMR} \mathrm{(471} \mathrm{MHz}, \mathrm{CDCl}_{3}$ ) of $2 \mathrm{k}$ :

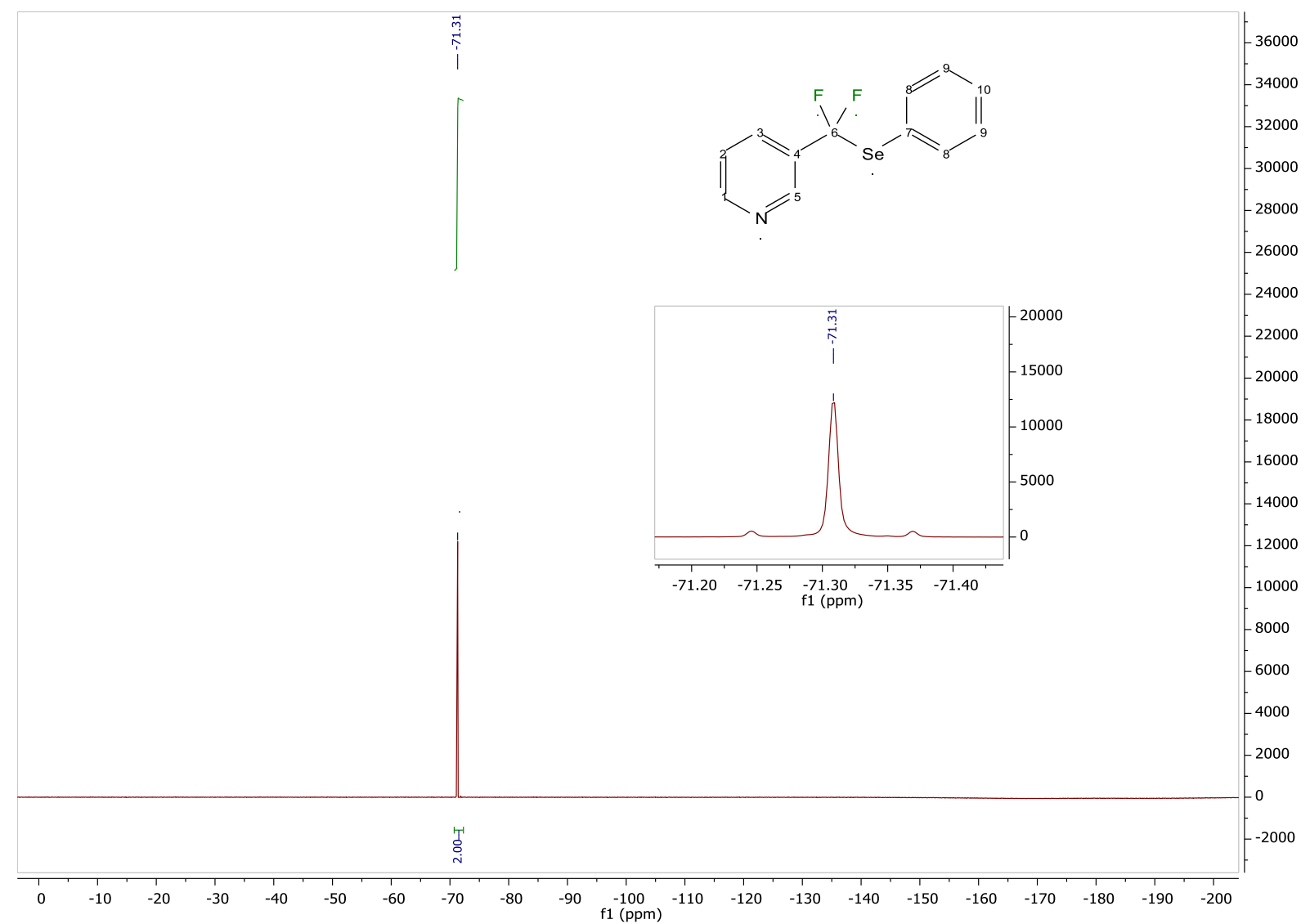




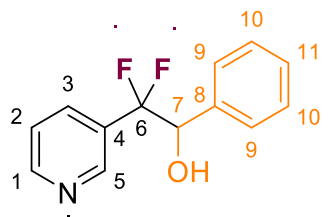

By sequential quench:

2I was prepared according to protocol A using benzaldehyde from 1 to 5 equivalents. $0 \%$ of ${ }^{19} \mathrm{~F} \mathrm{NMR}$ yield was calculated with fluorobenzene as internal standard.

\section{By desilylative post-functionalization:}

Benzaldehyde (1 equiv., $0.4 \mathrm{mmol}, 0.04 \mathrm{~mL}$ ) was added to a solution of 3((dimethyl(phenyl)silyl)difluoromethyl)pyridine $\mathbf{2 b}$ (1 equiv., $0.4 \mathrm{mmol}, 109 \mathrm{mg}$ ) in DMF $(2 \mathrm{~mL})$ at $0{ }^{\circ} \mathrm{C}$ and under inert atmosphere. A solution of TBAT (0.1 equiv., $0.04 \mathrm{mmol}, 21 \mathrm{mg}$ ) in DMF (4 mL) precooled to $0{ }^{\circ} \mathrm{C}$ was added to the reaction mixture, which was then stirred for $30 \mathrm{~min}$ at $20^{\circ} \mathrm{C}$. TBAF (1 equiv., $1.0 \mathrm{M}$ in THF, $0.38 \mathrm{mmol}, 0.11 \mathrm{~mL}$ ) was added to obtain the desilylated alcohol $21.99 \%$ of ${ }^{19} \mathrm{~F}$ NMR yield was calculated with fluorobenzene as internal standard. Purification via flash column chromatography (silica gel, gradient from 0 to $40 \%$ of EtOAc in cyclohexane) yielded $2 \mathbf{2}$ as a white crystalline solid ( $75 \mathrm{mg}, 0.32 \mathrm{mmol}, 81 \%$ ).

${ }^{1} \mathrm{H}$ NMR (400 MHz, $\left.\mathrm{CDCl}_{3}\right): \delta 8.63(\mathrm{~d}, J=4.9 \mathrm{~Hz}, 1 \mathrm{H}, \mathrm{H} 1), 8.48(\mathrm{~s}, 1 \mathrm{H}, \mathrm{H} 5), 7.50(\mathrm{dt}, J=7.9,2.2 \mathrm{~Hz}, 1 \mathrm{H}$, $\mathrm{H} 3), 7.33-2.23\left(\mathrm{~m}, 4 \mathrm{H}, \mathrm{H} 2, \mathrm{H}_{\mathrm{Ar}}\right), 7.18\left(\mathrm{~m}, 2 \mathrm{H}, \mathrm{H}_{\mathrm{Ar}}\right), 5.14\left(\mathrm{t}, \mathrm{H}-\mathrm{F},{ }^{3}{ }_{\mathrm{H}-\mathrm{F}}=9.1 \mathrm{~Hz}, 1 \mathrm{H}, \mathrm{H} 7\right), 2.76$ (brs, $1 \mathrm{H}$, $\mathrm{OH}$.

${ }^{13} \mathrm{C}$ NMR $\left(101 \mathrm{MHz}, \mathrm{CDCl}_{3}\right): \delta 151.0(\mathrm{C} 1), 147.8\left(\mathrm{t}, \mathrm{C}-\mathrm{F},{ }^{3} \mathrm{~J}_{\mathrm{C}-\mathrm{F}}=6.6 \mathrm{~Hz}, \mathrm{C} 5\right), 135.5(\mathrm{C} 8), 134.6\left(\mathrm{t}, \mathrm{C}-\mathrm{F},{ }^{3} \mathrm{~J}_{\mathrm{C}-\mathrm{F}}=\right.$ $6.1 \mathrm{~Hz}, \mathrm{C} 3), 129.8\left(\mathrm{t}, \mathrm{C}-\mathrm{F},{ }^{2} \mathrm{~J}_{\mathrm{C}-\mathrm{F}}=26.8 \mathrm{~Hz}, \mathrm{C} 4\right), 129.1\left(\mathrm{C}_{\mathrm{Ar}}\right), 128.3\left(\mathrm{C}_{\mathrm{Ar}}\right), 127.7\left(\mathrm{C}_{\mathrm{Ar}}\right), 122.7(\mathrm{C} 2), 120.5(\mathrm{t}, \mathrm{C}-$ $\left.F,{ }^{1} J_{C-F}=248.3 \mathrm{~Hz}, C 6\right), 76.5(C 7)$.

${ }^{19} \mathrm{~F}$ NMR $\left(377 \mathrm{MHz}, \mathrm{CDCl}_{3}\right): \delta-105.32\left(\mathrm{dd},{ }^{2} J_{\mathrm{F}-\mathrm{F}}=255.5 \mathrm{~Hz},{ }^{3} \mathrm{~J}_{\mathrm{F}-\mathrm{H}}=9.5 \mathrm{~Hz}, 1 \mathrm{~F}\right),-107.19\left(\mathrm{dd},{ }^{2} J_{\mathrm{F}-\mathrm{F}}=255.0\right.$ $\left.\mathrm{Hz},{ }^{3} \mathrm{JF}_{\mathrm{F}-\mathrm{H}}=8.6 \mathrm{~Hz}, 1 \mathrm{~F}\right)$.

HRMS (ESI): $\mathrm{m} / \mathrm{z}[\mathrm{M}+\mathrm{H}]^{+}$calcd for $\mathrm{C}_{13} \mathrm{H}_{12} \mathrm{~F}_{2} \mathrm{NO}$ : 236.0881; found: 236.0883 .

FTIR (cm ${ }^{-1}$, neat): 3066, 1600, 1426, 1345, 1132, 1064, 1022, 711.

These data are in accordance with previously reported results. ${ }^{6}$ 
${ }^{1} \mathrm{H}$ NMR (400 MHz, $\mathrm{CDCl}_{3}$ ) of 2l:

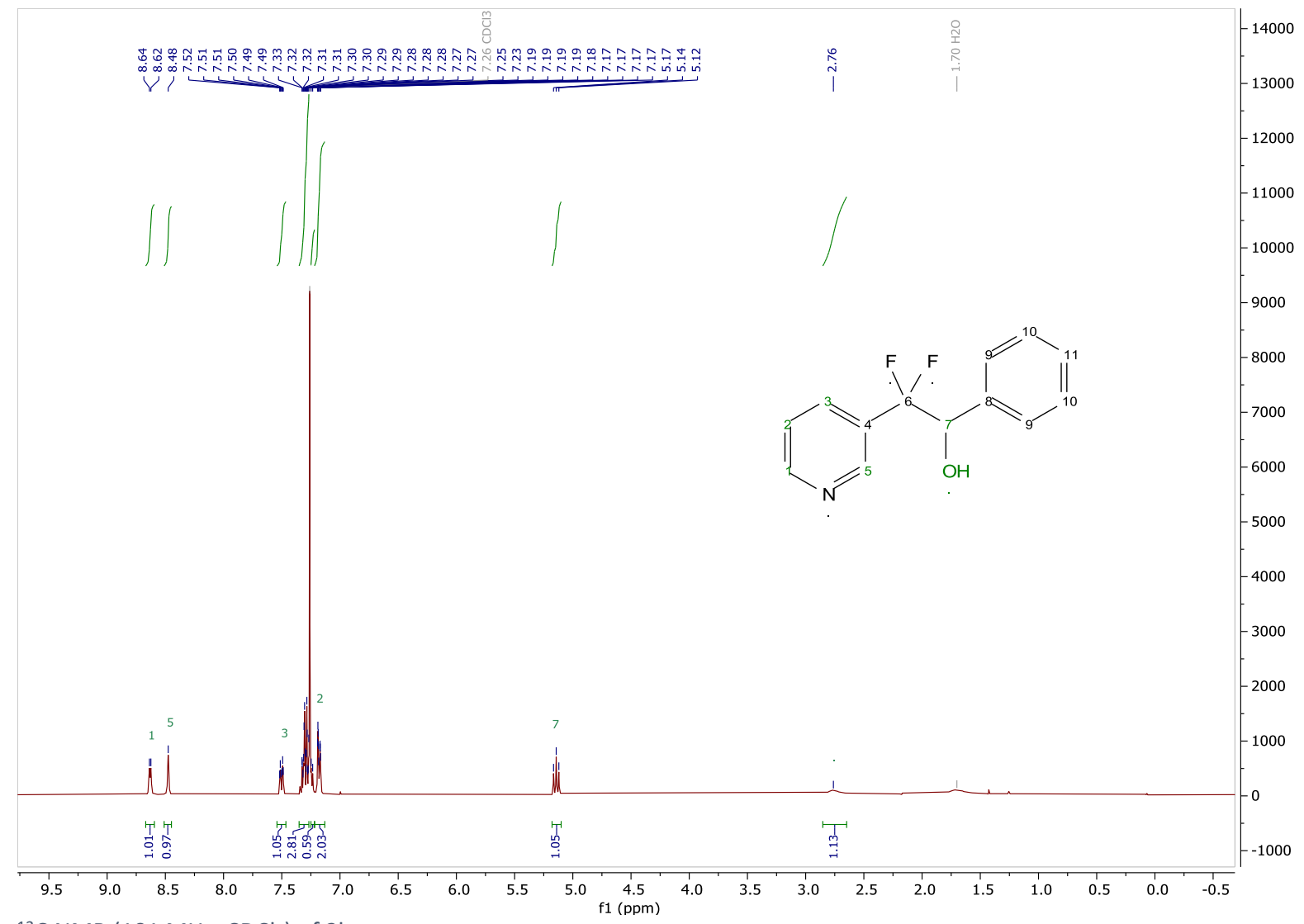

${ }^{13} \mathrm{C}$ NMR (101 MHz, $\mathrm{CDCl}_{3}$ ) of 2l:

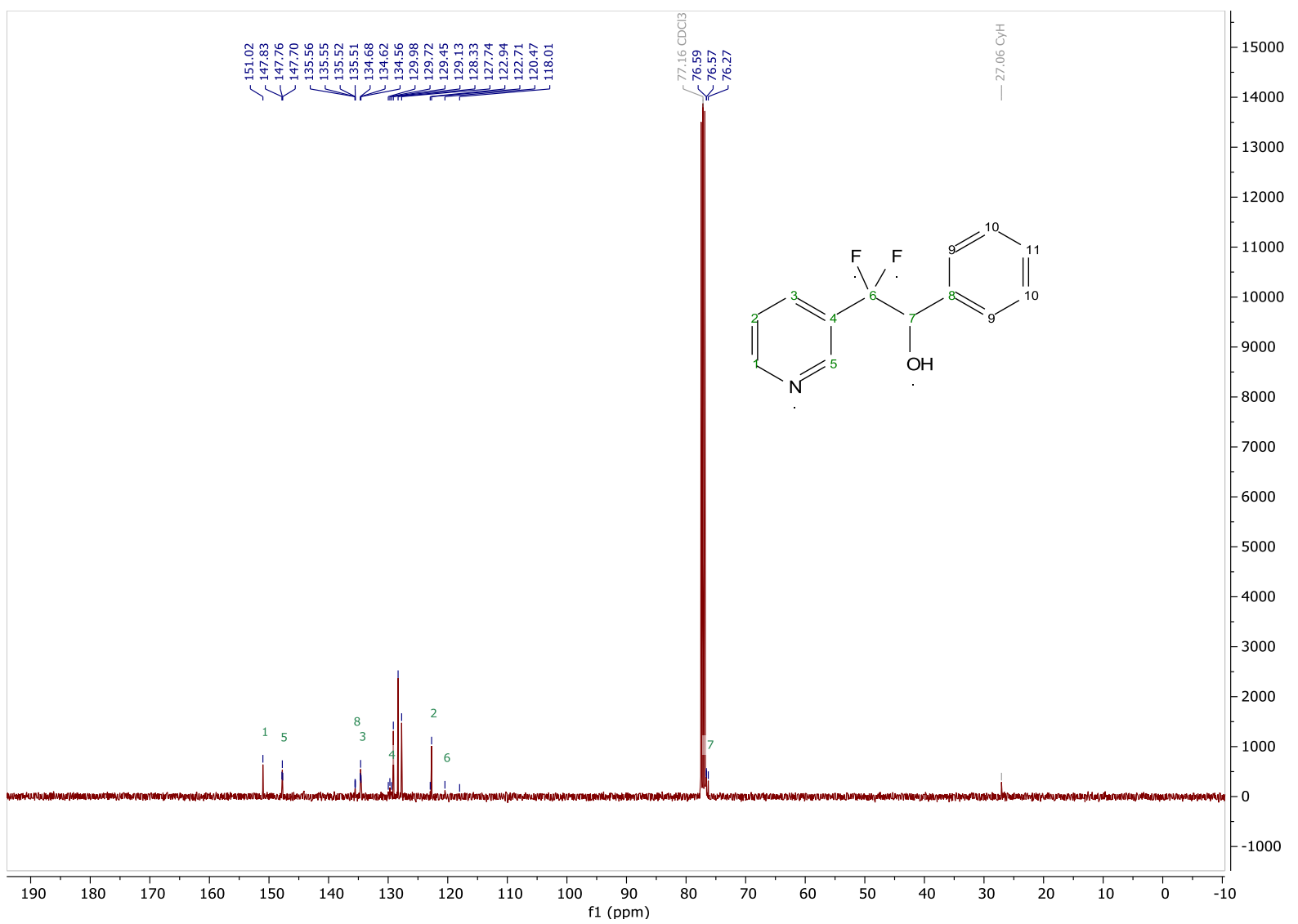




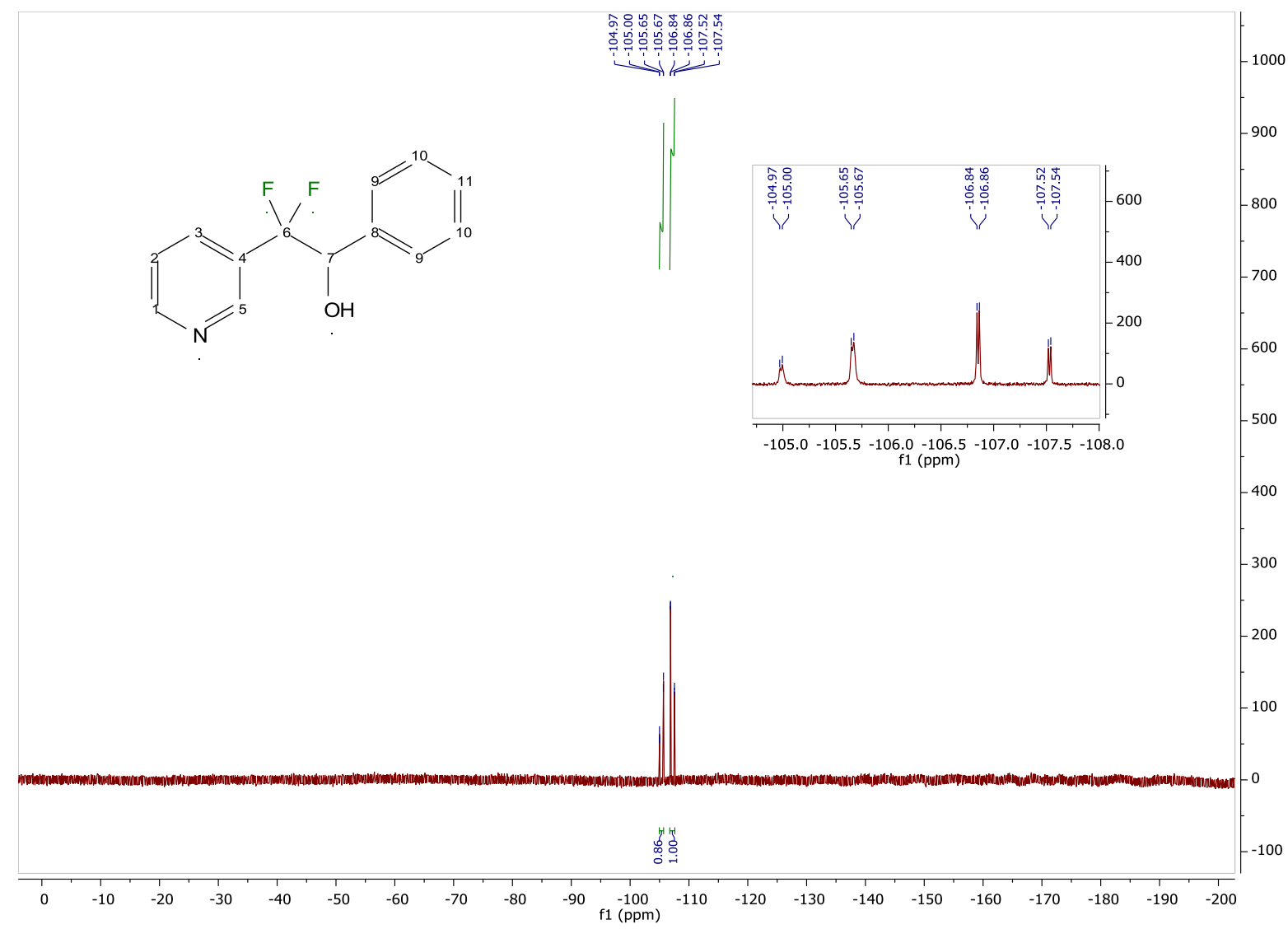

1-(4-chlorophenyl)-2,2-difluoro-2-(pyridin-3-yl)ethan-1-ol (2m)<smiles>OC(c1ccc(Cl)cc1)C(F)(F)c1cccnc1</smiles>

\section{By sequential quench:}

$\mathbf{2 m}$ was prepared according to protocol A using $p$-chloro-benzaldehyde from 1 to 5 equivalents. $0 \%$ of ${ }^{19} \mathrm{~F}$ NMR yield was calculated with fluorobenzene as internal standard.

\section{By desilylative post-functionalization:}

p-chloro-benzaldehyde (1 equiv., $0.23 \mathrm{mmol}, 32 \mathrm{mg}$ ) was added to a solution of 3((dimethyl(phenyl)silyl)difluoromethyl)pyridine $\mathbf{2 b}$ (1 equiv., $0.23 \mathrm{mmol}, 60 \mathrm{mg}$ ) in DMF $(1.1 \mathrm{~mL}$ ) at 0 ${ }^{\circ} \mathrm{C}$ and under inert atmosphere. A solution of TBAT (0.1 equiv., $0.023 \mathrm{mmol}, 12 \mathrm{mg}$ ) in DMF $(0.6 \mathrm{~mL})$ pre-cooled to $0{ }^{\circ} \mathrm{C}$ was added to the reaction mixture, which was then stirred for $30 \mathrm{~min}$ at $20^{\circ} \mathrm{C}$. TBAF (1 equiv., $1.0 \mathrm{M}$ in THF, $0.23 \mathrm{mmol}, 0.23 \mathrm{~mL}$ ) was added to obtain the desilylated alcohol $2 \mathrm{~m}$. $72 \%$ of ${ }^{19} \mathrm{~F}$ NMR yield was calculated with fluorobenzene as internal standard. Purification via flash column chromatography (silica gel, gradient from 0 to $40 \%$ of EtOAc in cyclohexane) yielded $2 \mathrm{~m}$ as a white amorphous solid (39 mg, $0.14 \mathrm{mmol}, 63 \%$ ).

${ }^{1} \mathrm{H}$ NMR (400 MHz, CDCl$)$ ): $\delta 8.63(\mathrm{dd}, J=4.3 \mathrm{~Hz}, 1 \mathrm{H}, \mathrm{H} 1), 8.51(\mathrm{~d}, J=1.8 \mathrm{~Hz}, 1 \mathrm{H}, \mathrm{H} 5), 7.58(\mathrm{dt},, J=8.0$, $2.0 \mathrm{~Hz} 1 \mathrm{H}, \mathrm{H} 3), 7.35-7.31(\mathrm{~m}, 3 \mathrm{H}, \mathrm{H} 2, \mathrm{H} 9), 7.21(\mathrm{~d}, \mathrm{~J}=8.2 \mathrm{~Hz}, 2 \mathrm{H}, \mathrm{H} 10), 5.18\left(\mathrm{t}, \mathrm{H}-\mathrm{F},{ }^{3} \mathrm{~J}_{\mathrm{H}-\mathrm{F}}=8.9 \mathrm{~Hz}, 1 \mathrm{H}\right.$, $\mathrm{H} 7), 4.52$ (brs, $1 \mathrm{H}, \mathrm{OH})$. 
${ }^{13} \mathrm{C}$ NMR (101 MHz, CDCl 3 ): $\delta 150.8$ (C1), 147.6 (t, C-F, $\left.{ }^{3} \mathrm{~J}_{\mathrm{C}-\mathrm{F}}=6.5 \mathrm{~Hz}, \mathrm{C} 5\right), 134.9$ (C11), 134.8 (t, C-F, ${ }^{3} \mathrm{~J}_{\mathrm{C}-\mathrm{F}}$ $=6.1 \mathrm{~Hz}, \mathrm{C} 3), 134.4\left(\mathrm{t}, \mathrm{C}-\mathrm{F},{ }^{3} \mathrm{~J}_{\mathrm{C}-\mathrm{F}}=4.3 \mathrm{~Hz}, \mathrm{C} 8\right), 129.7\left(\mathrm{t}, \mathrm{C}-\mathrm{F},{ }^{2} \mathrm{~J}_{\mathrm{C}-\mathrm{F}}=26.5 \mathrm{~Hz}, \mathrm{C} 4\right), 129.1$ (C9), 128.5 (C10), $122.9(\mathrm{C} 2), 120.3\left(\mathrm{t}, \mathrm{C}-\mathrm{F},{ }^{1} \mathrm{~J}_{\mathrm{C}-\mathrm{F}}=248.7 \mathrm{~Hz}, \mathrm{C} 6\right), 75.6\left(\mathrm{t}, \mathrm{C}-\mathrm{F},{ }^{2} \mathrm{~J}_{\mathrm{C}-\mathrm{F}}=30.6 \mathrm{~Hz}, \mathrm{C}\right.$ ) $)$.

${ }^{19} \mathrm{~F} \mathrm{NMR}\left(471 \mathrm{MHz}, \mathrm{CDCl}_{3}\right): \delta-104.02\left(\mathrm{dd},{ }^{2} \mathrm{~J}_{\mathrm{F}-\mathrm{F}}=255.8 \mathrm{~Hz},{ }^{3} \mathrm{~J}_{\mathrm{F}-\mathrm{H}}=8.9 \mathrm{~Hz}, 1 \mathrm{~F}\right),-107.72\left(\mathrm{dd},{ }^{2} \mathrm{~J}_{\mathrm{F}-\mathrm{F}}=255.78\right.$ $\left.\mathrm{Hz},{ }^{3} \mathrm{~J}_{\mathrm{F}-\mathrm{H}}=9.2 \mathrm{~Hz}, 1 \mathrm{~F}\right)$.

HRMS (ESI): $\mathrm{m} / \mathrm{z}[\mathrm{M}+\mathrm{H}]^{+}$calcd for $\mathrm{C}_{13} \mathrm{H}_{11} \mathrm{ClF}_{2} \mathrm{NO}: 270.0492$; found: 270.0493.

FTIR (cm ${ }^{-1}$, neat): 2860, 1588, 1574, 1485, 1385, 1283, 1206, 1090, 101, 838, 815.

${ }^{1} \mathrm{H}$ NMR (400 MHz, $\mathrm{CDCl}_{3}$ ) of $2 \mathrm{~m}$ :

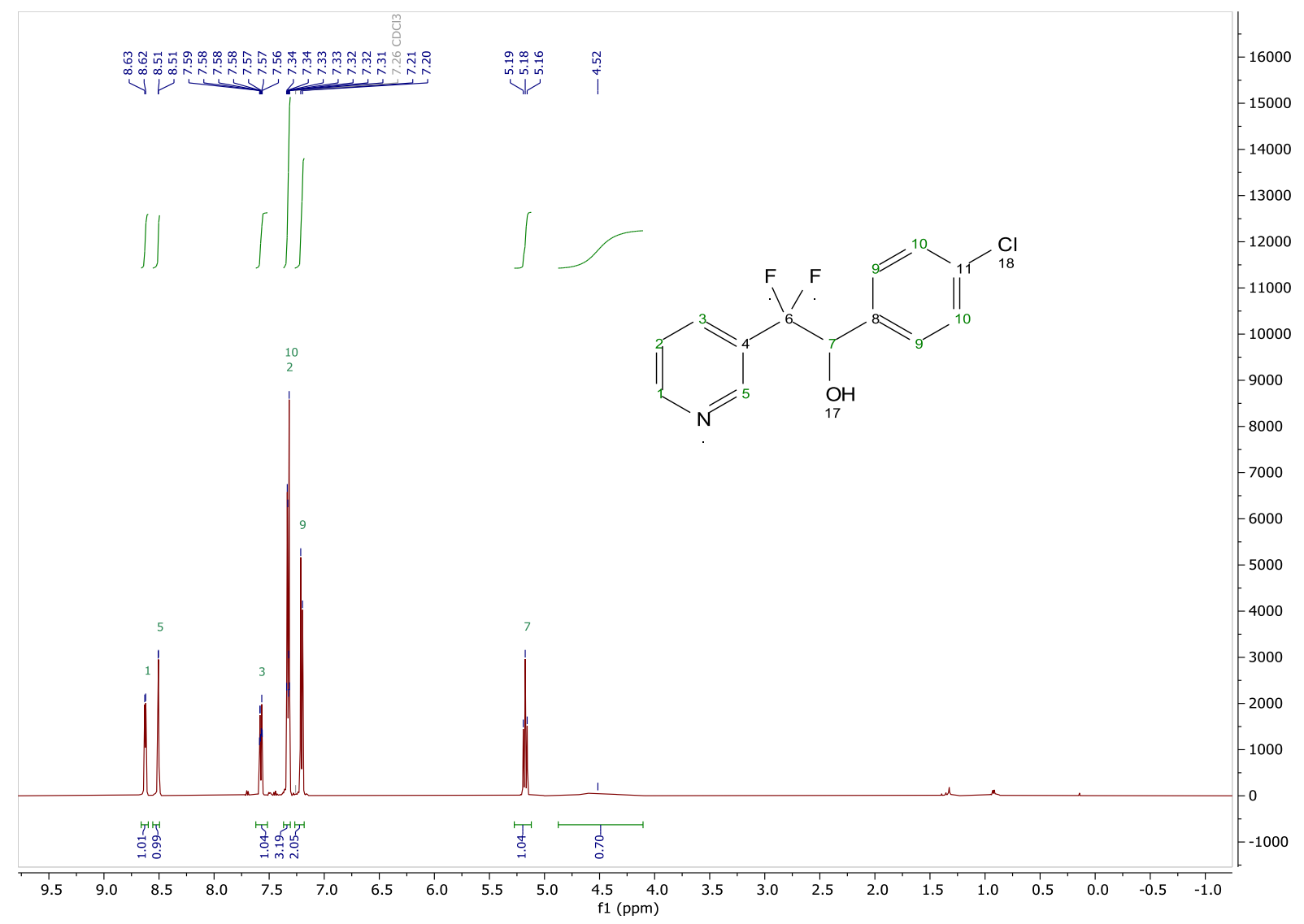


${ }^{13} \mathrm{C}$ NMR (101 MHz, $\mathrm{CDCl}_{3}$ ) of $2 \mathrm{~m}$ :

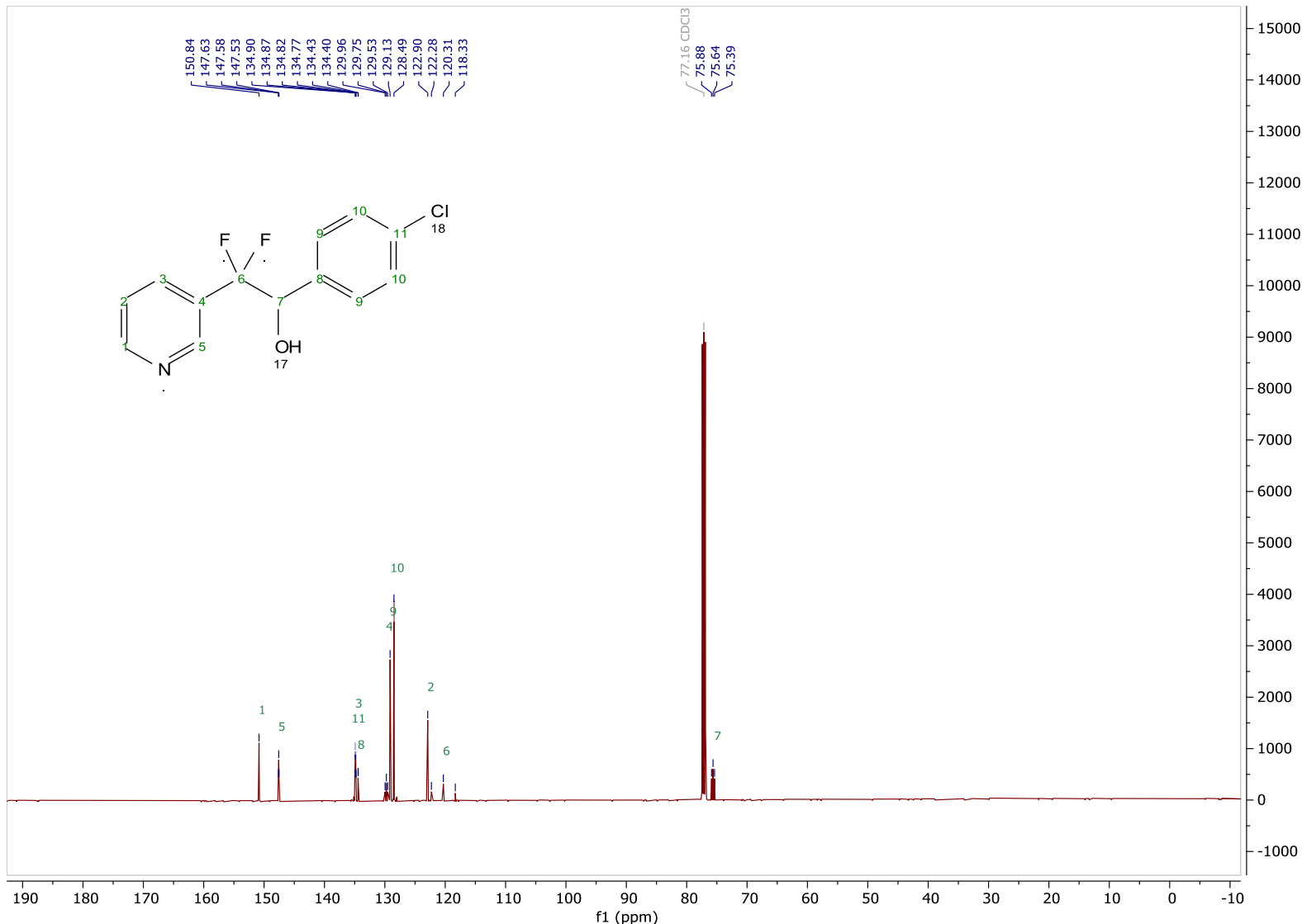

${ }^{19} \mathrm{~F}$ NMR (471 MHz, $\mathrm{CDCl}_{3}$ ) of $2 \mathrm{~m}$ :

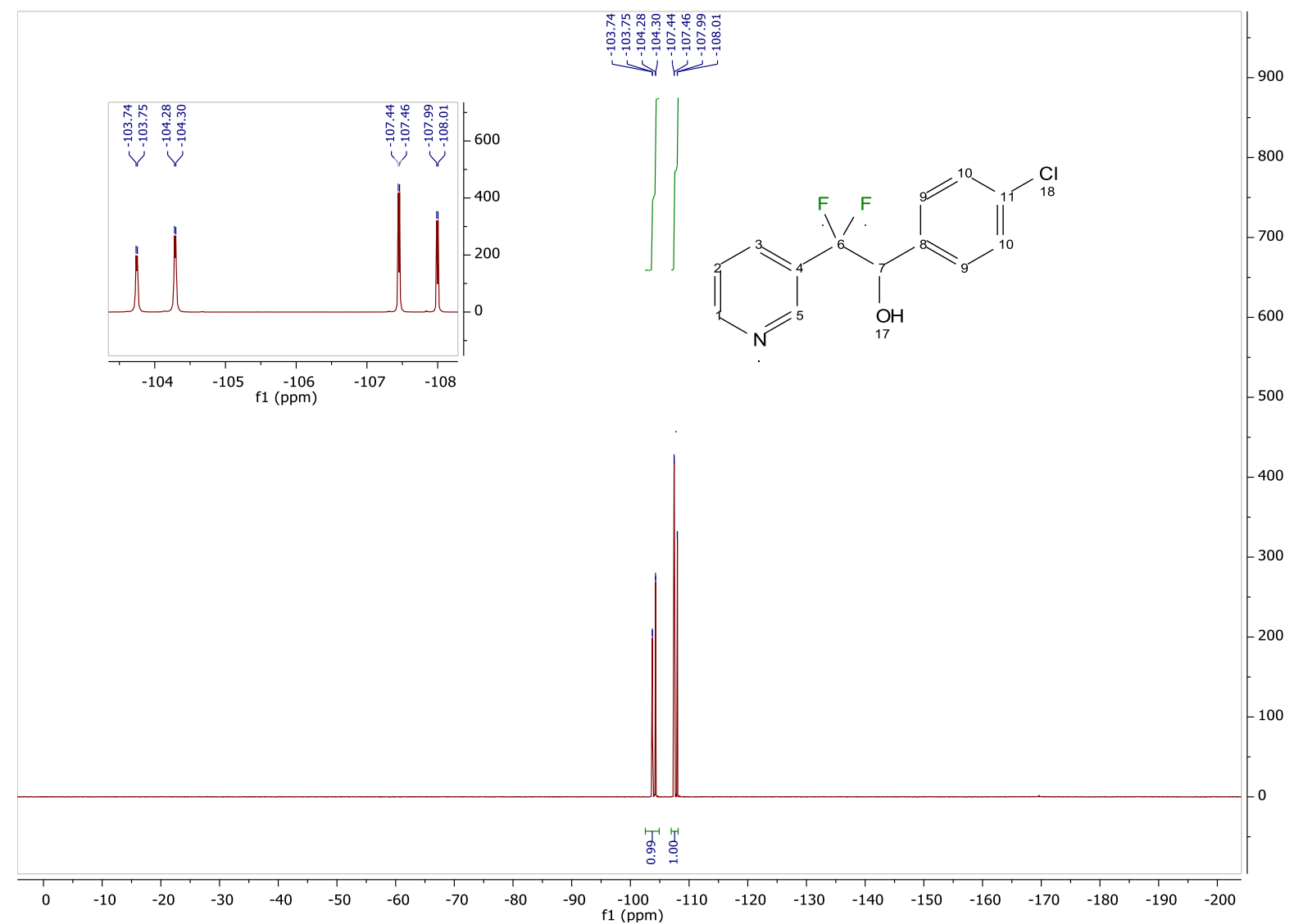




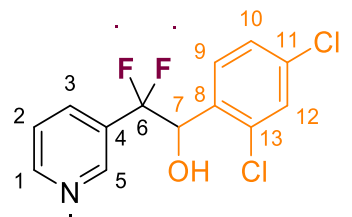

By sequential quench:

2n was prepared according to protocol A using 1 equivalent of 2,4-dichloro-benzaldehyde. $0 \%$ of ${ }^{19} \mathrm{~F}$ NMR yield was calculated with fluorobenzene as internal standard.

\section{By desilylative post-functionalization:}

2,4-dichloro-benzaldehyde (1.1 equiv., $1.46 \mathrm{mmol}, 256 \mathrm{mg}$ ) was added to a solution of 3((dimethyl(phenyl)silyl)difluoromethyl)pyridine $\mathbf{2 b}$ (1 equiv., $1.33 \mathrm{mmol}, 350 \mathrm{mg}$ ) in DMF $(6 \mathrm{~mL})$ at $0{ }^{\circ} \mathrm{C}$ and under inert atmosphere. A solution of TBAT (0.1 equiv., $0.13 \mathrm{mmol}, 72 \mathrm{mg}$ ) in DMF ( $3 \mathrm{~mL}$ ) precooled to $0{ }^{\circ} \mathrm{C}$ was added to the reaction mixture, which was then stirred for $1 \mathrm{~h}$ at $20{ }^{\circ} \mathrm{C}$. TBAF (1 equiv., $1.0 \mathrm{M}$ in THF, $1.33 \mathrm{mmol}, 1.3 \mathrm{~mL}$ ) was added to obtain the desilylated alcohol $2 \mathrm{n} .80 \%$ of ${ }^{19} \mathrm{~F}$ NMR yield was calculated with fluorobenzene as internal standard. Purification via flash column chromatography (silica gel, gradient from 0 to $40 \%$ of EtOAc in cyclohexane) yielded $\mathbf{2 n}$ as a white amorphous solid ( $275 \mathrm{mg}, 0.90 \mathrm{mmol}, 68 \%$ ).

${ }^{1} \mathrm{H}$ NMR (400 MHz, $\left.\mathrm{CDCl}_{3}\right): 8.70-8.62(\mathrm{~m}, 1 \mathrm{H}, \mathrm{H} 1), 8.55(\mathrm{~d}, J=2.4 \mathrm{~Hz}, 1 \mathrm{H}, \mathrm{H} 5), 7.61(\mathrm{dt}, J=8.0,2.0 \mathrm{~Hz}$, $1 \mathrm{H}, \mathrm{H} 3), 7.36$ (d, J = 8.5 Hz, 1H, H9), 7.34 (d, J=2.1 Hz, 1H, H12), $7.32-7.29(\mathrm{~m}, 1 \mathrm{H}, \mathrm{H} 2), 7.25$ (dd, $J=$ 8.5, 2.2 Hz, 1H, H10), 5.61 (td, J = 9.7, $4.5 \mathrm{~Hz}, 1 \mathrm{H}, \mathrm{H7}$ ), 3.26 (d, J = 4.5 Hz, $1 \mathrm{H}, \mathrm{OH}$ ).

${ }^{13} \mathrm{C}$ NMR (101 MHz, CDCl $)$ : $\delta 151.4$ (C1), $147.8\left(\mathrm{t}, \mathrm{C}-\mathrm{F},{ }^{3} \mathrm{~J}_{\mathrm{C}-\mathrm{F}}=6.5 \mathrm{~Hz}, \mathrm{C} 5\right), 135.6$ (C11), 134.6 (C13), 134.4 (t, C-F, $\left.{ }^{3} J_{C-F}=5.9 \mathrm{~Hz}, C 3\right), 132.2$ (C8), 130.7 (C9), 129.8 (t, C-F, $\left.{ }^{2} J_{C-F}=26.3 \mathrm{~Hz}, \mathrm{C} 4\right), 129.3$ (C12), 127.5 (C10), 123.0 (C2), $120.2\left(\mathrm{t}, \mathrm{C}-\mathrm{F},{ }^{1} \mathrm{~J}_{\mathrm{C}-\mathrm{F}}=250.0 \mathrm{~Hz}, \mathrm{C} 6\right), 72.0\left(\mathrm{t}, \mathrm{C}-\mathrm{F},{ }^{2} \mathrm{~J}_{\mathrm{C}-\mathrm{F}}=30.7 \mathrm{~Hz}, \mathrm{C} 7\right)$.

${ }^{19} \mathrm{~F} \mathrm{NMR}\left(471 \mathrm{MHz}, \mathrm{CDCl}_{3}\right): \delta-106.41\left(\mathrm{dd},{ }^{2} \mathrm{~J}_{\mathrm{F}-\mathrm{F}}=254.0 \mathrm{~Hz},{ }^{3} \mathrm{~J}_{\mathrm{F}-\mathrm{H}}=10.4 \mathrm{~Hz}, 1 \mathrm{~F}\right),-109.44\left(\mathrm{dd},{ }^{2} \mathrm{~J}_{\mathrm{F}-\mathrm{F}}=254.3\right.$ $\left.\mathrm{Hz},{ }^{3} \mathrm{JF}_{\mathrm{F}-\mathrm{H}}=9.1 \mathrm{~Hz}, 1 \mathrm{~F}\right)$.

HRMS (ESI): $\mathrm{m} / \mathrm{z}[\mathrm{M}+\mathrm{H}]^{+}$calcd for $\mathrm{C}_{13} \mathrm{H}_{10} \mathrm{Cl}_{2} \mathrm{~F}_{2} \mathrm{NO}$ : 304.0102; found: 304.0090 . 
${ }^{1} \mathrm{H}$ NMR $\left(400 \mathrm{MHz}, \mathrm{CDCl}_{3}\right)$ of $2 \mathrm{n}$ :

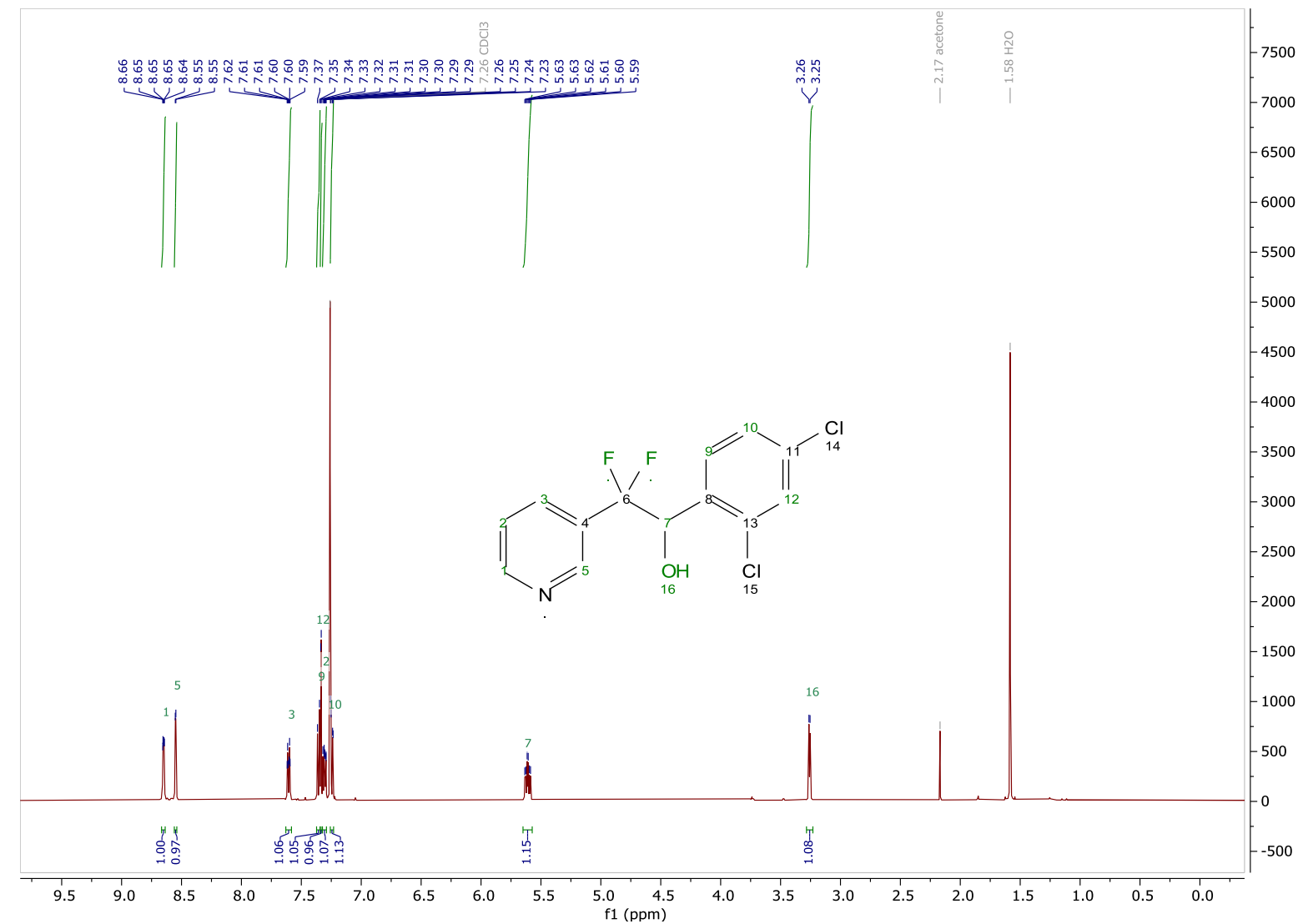

${ }^{13} \mathrm{C}$ NMR (101 MHz, $\mathrm{CDCl}_{3}$ ) of $2 \mathrm{n}$ :

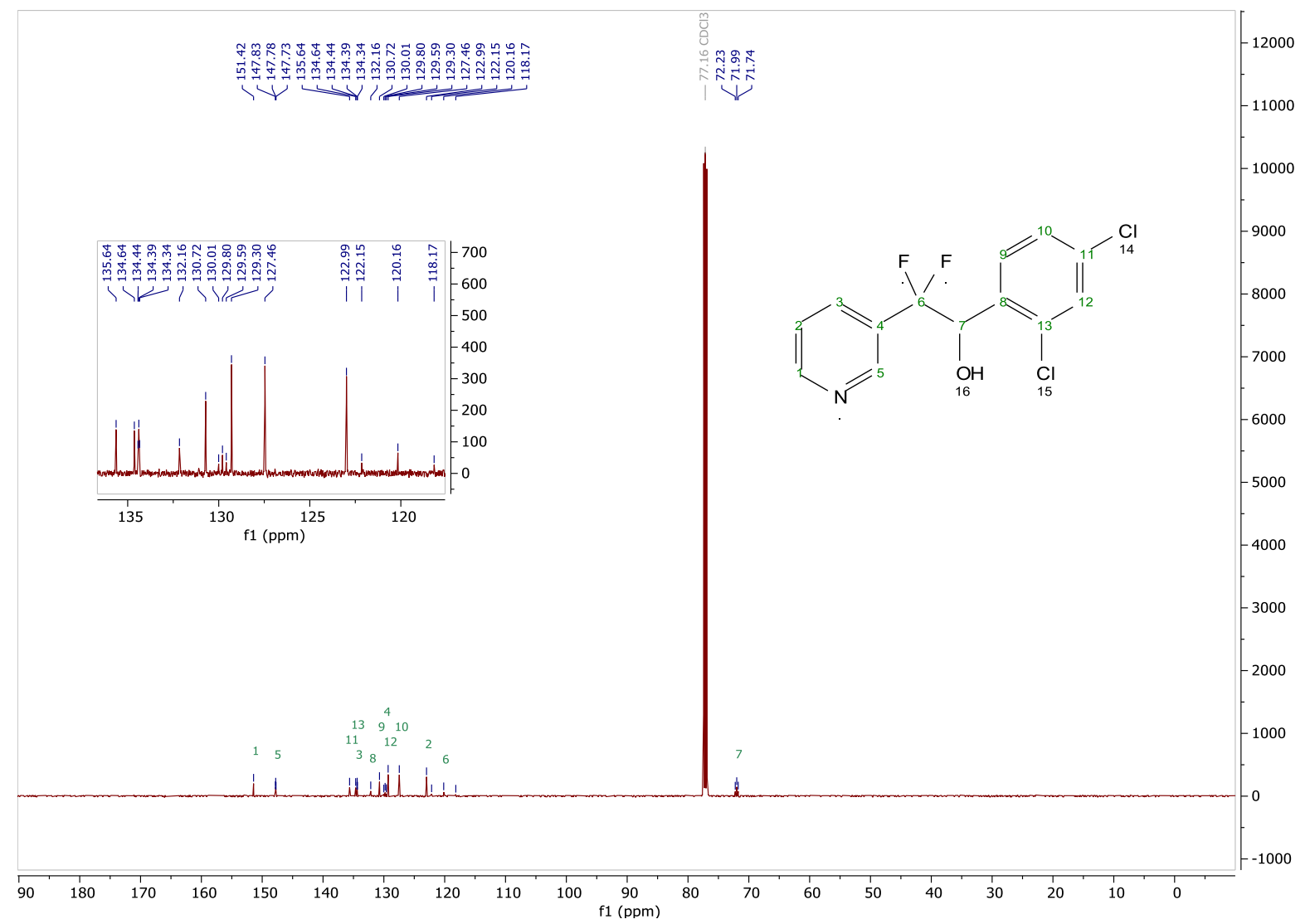




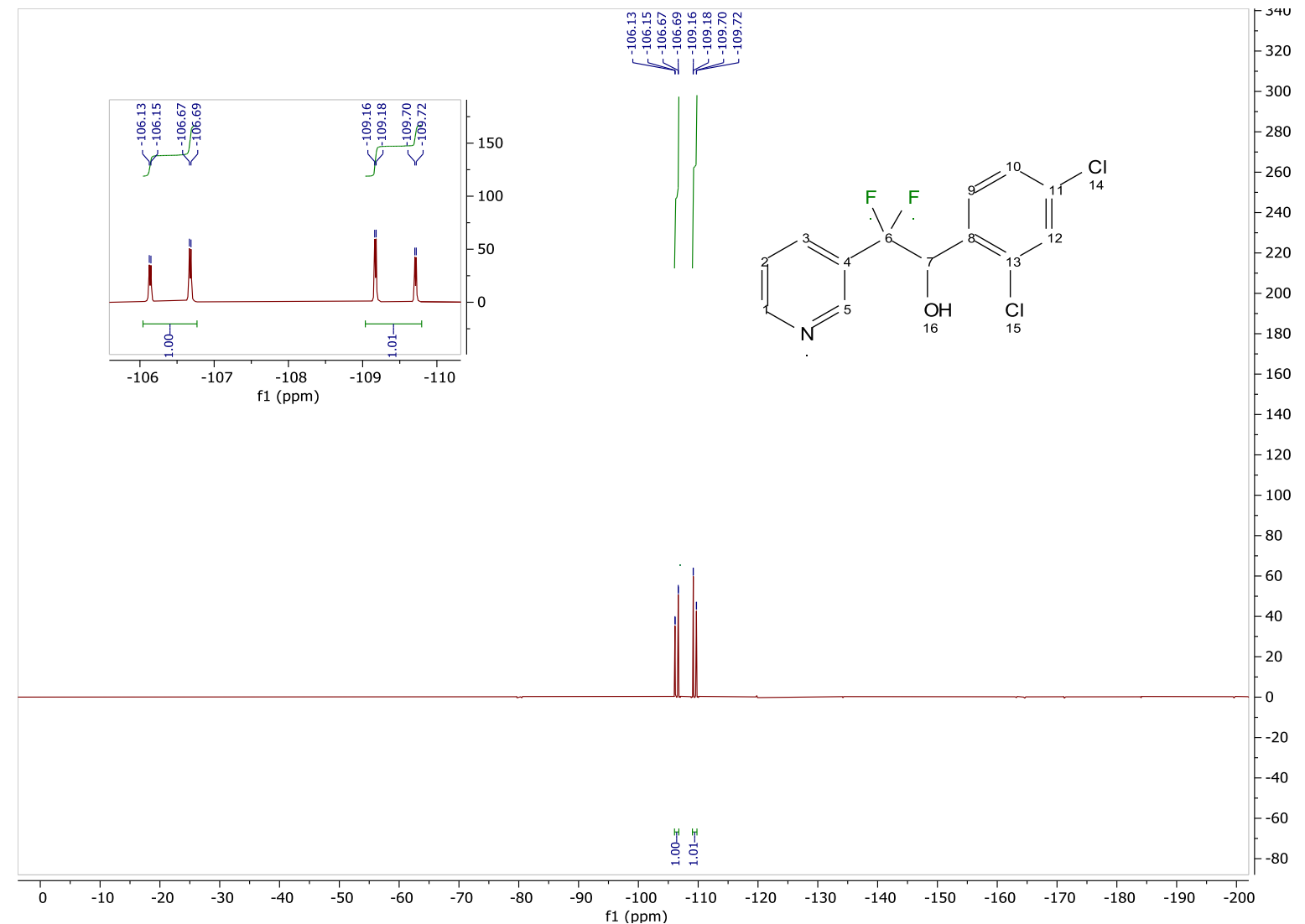

N-(2,2-difluoro-1-phenyl-2-(pyridin-3-yl)ethyl)-4-methylbenzenesulfonamide (20)

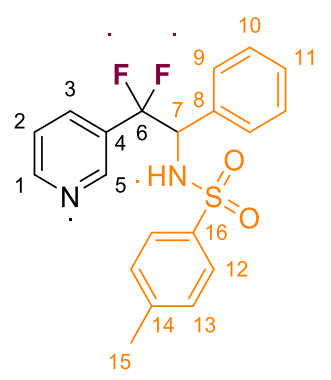

By sequential quench:

20 was prepared according to protocol A using 4-methyl- $N$-[(1Z)-phenylmethylidene]benzene-1sulfonamide (1 equiv., $3.85 \mathrm{mmol}, 996 \mathrm{mg}$ ). $0 \%$ of ${ }^{19} \mathrm{~F} \mathrm{NMR}$ yield was calculated with fluorobenzene as internal standard.

\section{By desilylative post-functionalization:}

4-methyl- $N$-[(1Z)-phenylmethylidene]benzene-1-sulfonamide (1 equiv., $0.21 \mathrm{mmol}, 55 \mathrm{mg}$ ) was added to a solution of 3-((dimethyl(phenyl)silyl)difluoromethyl)pyridine $\mathbf{2 b}$ (1 equiv., $0.23 \mathrm{mmol}, 60 \mathrm{mg}$ ) in DMF $\left(0.5 \mathrm{~mL}\right.$ ) at $0{ }^{\circ} \mathrm{C}$ and under inert atmosphere. A solution of TBAT (1 equiv., $0.22 \mathrm{mmol}, 117 \mathrm{mg}$ ) in DMF $(0.5 \mathrm{~mL})$ pre-cooled to $0{ }^{\circ} \mathrm{C}$ was added to the reaction mixture, which was then stirred for $14 \mathrm{~h}$ at $70{ }^{\circ} \mathrm{C} .47 \%$ of ${ }^{19} \mathrm{~F}$ NMR yield was calculated with fluorobenzene as internal standard. Purification via 
flash column chromatography (silica gel, gradient from 0 to $20 \%$ of EtOAc in cyclohexane) yielded 20 as a yellow oil ( $32 \mathrm{mg}, 0.083 \mathrm{mmol}, 39 \%$ ).

${ }^{1} \mathrm{H}$ NMR (400 MHz, CDCl 3$): \delta 9.31(\mathrm{~s}, 1 \mathrm{H}, \mathrm{H} 5), 9.12(\mathrm{~d}, J=5.5 \mathrm{~Hz}, 1 \mathrm{H}, \mathrm{H} 1), 8.46(\mathrm{~d}, J=10.8 \mathrm{~Hz}, 1 \mathrm{H}, \mathrm{H} 3)$, $7.95-7.86(\mathrm{~m}, 2 \mathrm{H}, \mathrm{H} 11, \mathrm{H} 2), 7.57(\mathrm{~d}, J=8.3,2 \mathrm{H}, \mathrm{H} 12), 7.28(\mathrm{~d}, J=7.7 \mathrm{~Hz}, 2 \mathrm{H}, \mathrm{H} 9), 7.23(\mathrm{~d}, J=6.7 \mathrm{~Hz}$, $1 \mathrm{H}, \mathrm{H} 3), 7.22-7.17(\mathrm{~m}, 2 \mathrm{H}, \mathrm{H} 10), 6.99(\mathrm{~d}, J=8.0 \mathrm{~Hz}, 2 \mathrm{H}, \mathrm{H} 13), 5.09$ (q, J = 10.3 Hz, 1H, H7), $2.24(\mathrm{~s}, 3 \mathrm{H}$, $\mathrm{H} 15)$.

${ }^{13} \mathrm{C}$ NMR (101 MHz, CDCl $): \delta 150.3$ (C1), 146.6 (C5), 143.6 (C16), 137.3 (C14), 135.3 (t, $\left.J=5.0 \mathrm{~Hz}, \mathrm{C} 3\right)$, $133.0(\mathrm{C} 8), 130.8\left(\mathrm{t}, \mathrm{C}-\mathrm{F},{ }^{2} \mathrm{~J}_{\mathrm{C}-\mathrm{F}}=26.0 \mathrm{~Hz}, \mathrm{C} 4\right), 129.5(\mathrm{C} 13), 128.7$ ( $\left.\mathrm{C}_{\mathrm{Ar}}\right), 128.4\left(\mathrm{C}_{\mathrm{Ar}}\right), 128.3\left(\mathrm{C}_{\mathrm{Ar}}\right), 127.0(\mathrm{C} 12)$, 123.6 (C2), $119.7\left(\mathrm{t}, \mathrm{C}-\mathrm{F},{ }^{1} J_{\mathrm{C}-\mathrm{F}}=250.7 \mathrm{~Hz}, \mathrm{C} 6\right), 63.3\left(\mathrm{t}, \mathrm{C}-\mathrm{F},{ }^{2} \mathrm{~J}_{\mathrm{C}-\mathrm{F}}=29.1 \mathrm{~Hz}, \mathrm{C} 7\right), 21.6$ (C15).

${ }^{19} \mathrm{~F}$ NMR (377 MHz, CDCl 3$): \delta-100.18\left(\mathrm{~d}, \mathrm{~F}-\mathrm{F},{ }^{3} \mathrm{~J}_{\mathrm{F}-\mathrm{F}}=257.0 \mathrm{~Hz}, 1 \mathrm{~F}\right),-103.08\left(\mathrm{~d}, \mathrm{~F}-\mathrm{F},{ }^{3} \mathrm{~J}_{\mathrm{F}-\mathrm{F}}=253.6 \mathrm{~Hz}, 1 \mathrm{~F}\right)$. HRMS (ESI): $\mathrm{m} / \mathrm{z}$; $[\mathrm{M}+\mathrm{H}]^{+}$calcd for $\mathrm{C}_{20} \mathrm{H}_{19} \mathrm{~F}_{2} \mathrm{~N}_{2} \mathrm{O}_{2} \mathrm{~S}$ : 389.1130; found: 389.1126 .

FTIR (cm ${ }^{-1}$, neat): 3068, 1599, 1492, 1458, 1426, 1332, 1161, 1091, 1075.

These data are in accordance with previously reported results. ${ }^{6}$

${ }^{1} \mathrm{H}$ NMR $\left(400 \mathrm{MHz}, \mathrm{CDCl}_{3}\right)$ of 20 :

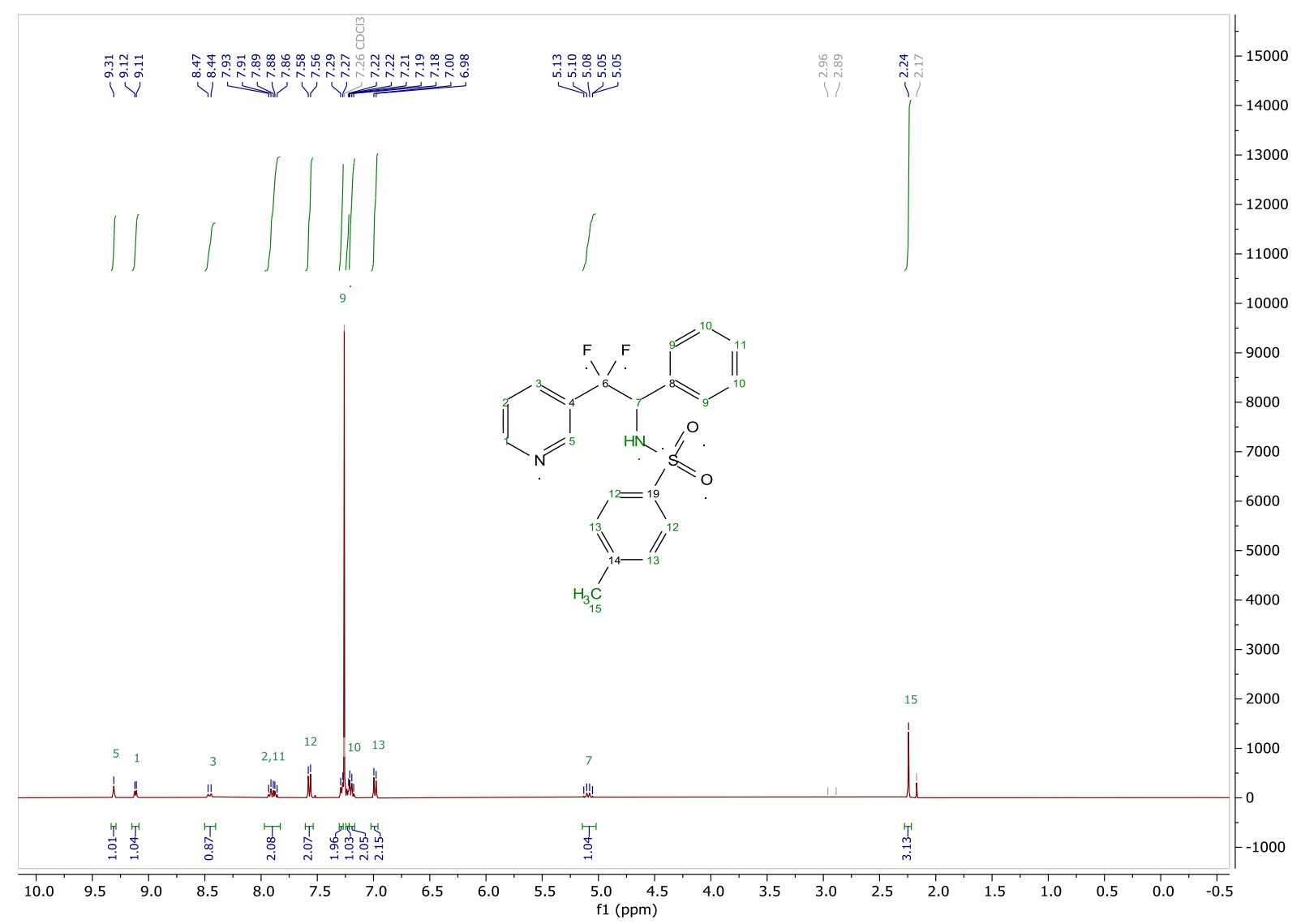


${ }^{13} \mathrm{C}$ NMR (101 MHz, $\mathrm{CDCl}_{3}$ ) of 2o:

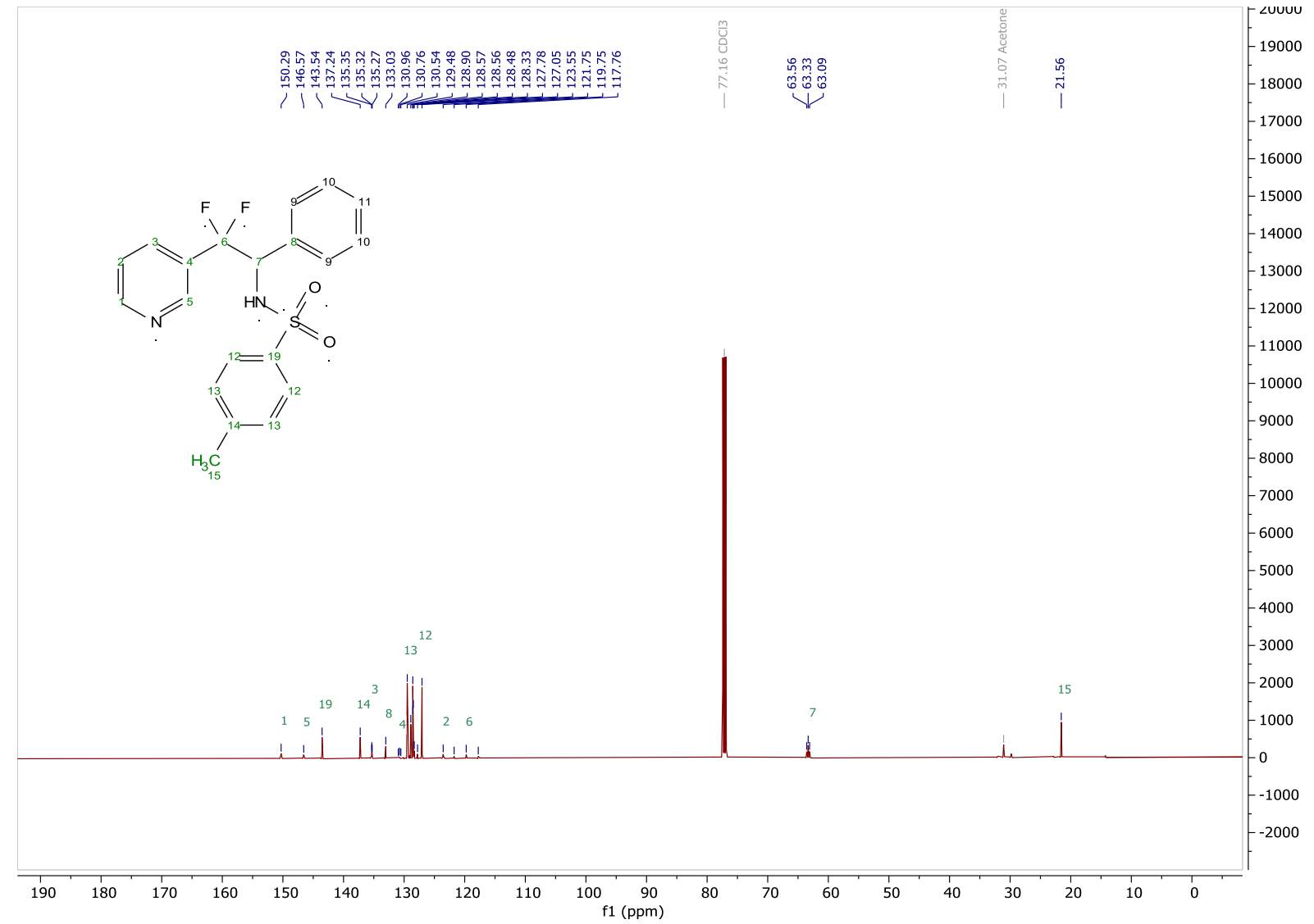

${ }^{19} \mathrm{~F} \mathrm{NMR} \mathrm{(377} \mathrm{MHz,} \mathrm{CDCl}_{3}$ ) of 20 :

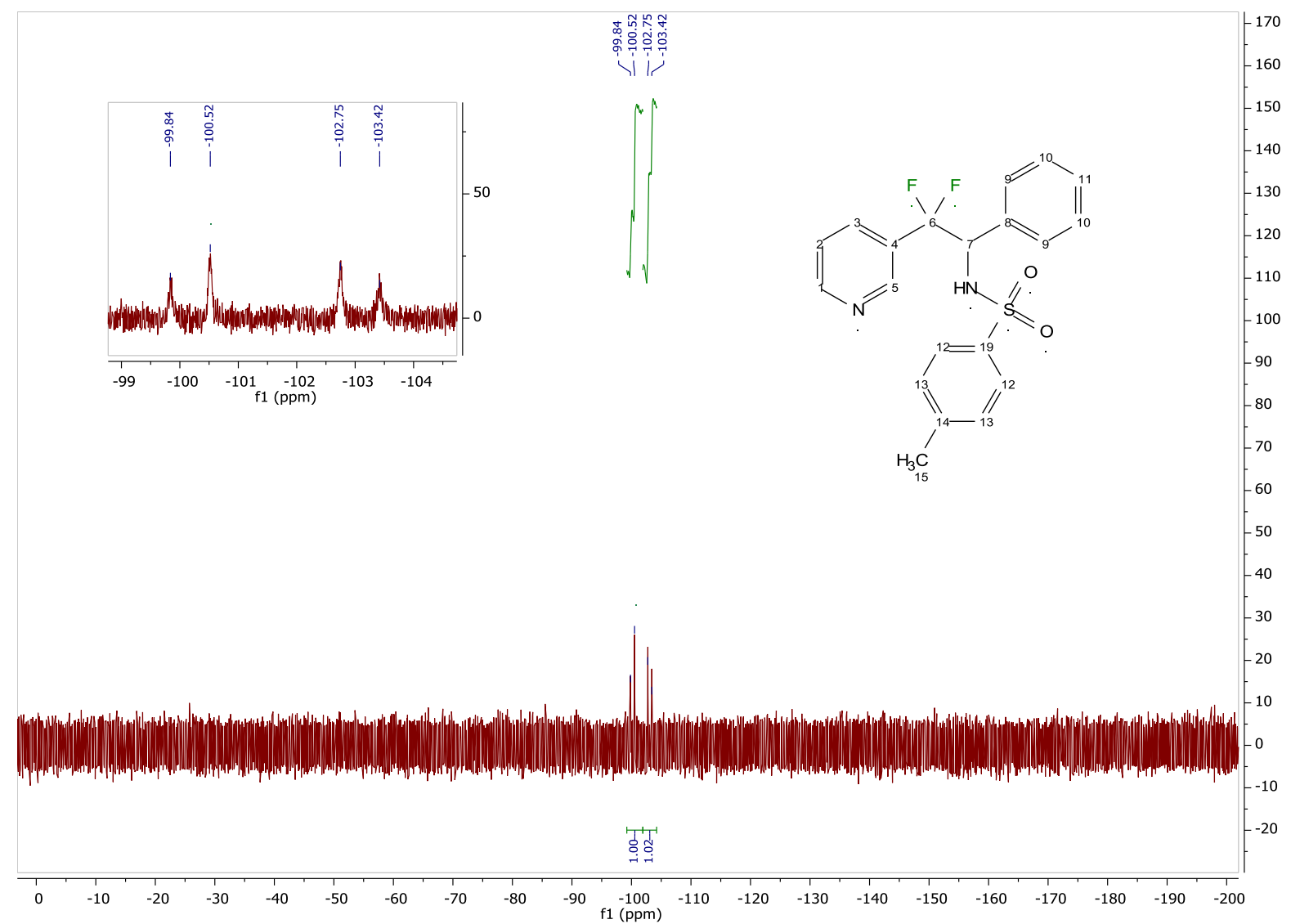




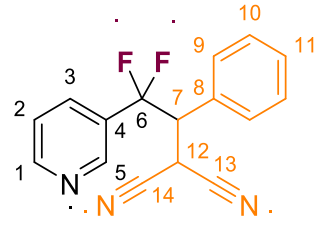

\section{By sequential quench:}

$2 \mathbf{p}$ was prepared according to protocol A using benzylidenemalononitrile (2 equiv., 3.85 mmol, 593 $\mathrm{mg}) .0 \%$ of ${ }^{19} \mathrm{~F} \mathrm{NMR}$ yield was calculated with fluorobenzene as internal standard.

\section{By desilylative post-functionalization:}

Benzylidenemalononitrile (1 equiv., $0.19 \mathrm{mmol}, 30 \mathrm{mg}$ ) was added to a solution of 3((dimethyl(phenyl)silyl)difluoromethyl)pyridine $\mathbf{2 b}$ (1 equiv., $0.18 \mathrm{mmol}, 47 \mathrm{mg}$ ) in DMF $(0.2 \mathrm{~mL}$ ) at 0 ${ }^{\circ} \mathrm{C}$ and under inert atmosphere. A solution of TBAT (1 equiv., $0.19 \mathrm{mmol}, 97 \mathrm{mg}$ ) in DMF $(0.2 \mathrm{~mL}$ ) precooled to $0{ }^{\circ} \mathrm{C}$ was added to the reaction mixture, which was then stirred for $4 \mathrm{~h}$ at $20{ }^{\circ} \mathrm{C} .28 \%$ of ${ }^{19} \mathrm{~F}$ NMR yield was calculated with fluorobenzene as internal standard. Purification via flash column chromatography (silica gel, gradient from 0 to $25 \%$ of EtOAc in cyclohexane) yielded $2 p$ as a colorless oil (19 mg, $0.035 \mathrm{mmol}, 20 \%)$.

${ }^{1} \mathrm{H}$ NMR (400 MHz, CDCl $): \delta 8.61(\mathrm{~d}, J=3.7 \mathrm{~Hz}, 1 \mathrm{H}, \mathrm{H} 1), 8.51(\mathrm{~s}, 1 \mathrm{H}, \mathrm{H} 5), 7.55-7.48(\mathrm{~m}, 1 \mathrm{H}, \mathrm{H} 3), 7.43$ $-7.32\left(\mathrm{~m}, 3 \mathrm{H}, \mathrm{H}_{\mathrm{Ar}}\right), 7.28\left(\mathrm{dt}, J=8.3,1.7 \mathrm{~Hz}, 2 \mathrm{H}, \mathrm{H}_{\mathrm{Ar}}\right), 7.22(\mathrm{dd}, J=8.1,4.9 \mathrm{~Hz}, 1 \mathrm{H}, \mathrm{H} 2), 4.64(\mathrm{~d}, J=6.1$ $\mathrm{Hz}, 1 \mathrm{H}, \mathrm{H} 12), 3.85$ (dt, $J=23.8,5.5 \mathrm{~Hz}, 1 \mathrm{H}, \mathrm{H} 7)$.

${ }^{13} \mathrm{C}$ NMR (101 MHz, CDCl 3$): \delta 152.0(\mathrm{C} 1), 146.9\left(\mathrm{t}, \mathrm{C}-\mathrm{F},{ }^{3} \mathrm{~J}_{\mathrm{C}-\mathrm{F}}=6.4 \mathrm{~Hz}, \mathrm{C5}\right), 133.3\left(\mathrm{t}, \mathrm{C}-\mathrm{F},{ }^{3} \mathrm{~J}_{\mathrm{C}-\mathrm{F}}=6.4 \mathrm{~Hz}, \mathrm{C} 3\right)$, $130.5\left(C_{A r}\right), 129.9\left(t, C-F,{ }^{2} J_{C-F}=26.8 \mathrm{~Hz}, C 4\right), 129.7\left(C_{A r}\right), 129.5\left(C_{A r}\right), 129.5\left(C_{A r}\right), 123.2(C 2), 120.7(d d$, $\left.\mathrm{C}-\mathrm{F},{ }^{1} J_{\mathrm{C}-\mathrm{F}}=253.9,250.0 \mathrm{~Hz}, \mathrm{C} 6\right), 111.3(\mathrm{C} 13), 110.9$ (C14), 54.7 (dd, C-F, $\left.{ }^{2} J_{\mathrm{C}-\mathrm{F}}=26.3,23.5 \mathrm{~Hz}, \mathrm{C} 7\right), 24.2$ (t, $\left.C-F,{ }^{3} J_{C-F}=3.4 \mathrm{~Hz}, C 12\right)$.

${ }^{19} \mathrm{~F}$ NMR $\left(377 \mathrm{MHz}, \mathrm{CDCl}_{3}\right): \delta-89.95\left(\mathrm{dd},{ }^{2} \mathrm{~J}_{\mathrm{F}-\mathrm{F}}=252.1 \mathrm{~Hz},{ }^{3} \mathrm{~J}_{\mathrm{F}-\mathrm{H}}=5.2 \mathrm{~Hz}, 1 \mathrm{~F}\right),-107.98\left(\mathrm{dd},{ }^{2} J_{\mathrm{F}-\mathrm{F}}=252.41\right.$ $\left.\mathrm{Hz},{ }^{3} \mathrm{~J}_{\mathrm{F}-\mathrm{H}}=23.8 \mathrm{~Hz}, 1 \mathrm{~F}\right)$.

HRMS (ESI): $\mathrm{m} / \mathrm{z}$; $[\mathrm{M}+\mathrm{H}]^{+}$calcd for $\mathrm{C}_{16} \mathrm{H}_{12} \mathrm{~F}_{2} \mathrm{LiN}_{3}$ : 290.1076; found: 290.1075 .

FTIR (cm ${ }^{-1}$, neat): 3054, 2918, 2263, 2208, 1596, 1495, 1457, 1425, 1285, 1160, 1074, 1049, 710. 
${ }^{1} \mathrm{H} \mathrm{NMR} \mathrm{(400} \mathrm{MHz,} \mathrm{CDCl}_{3}$ ) of $2 \mathrm{p}$ :

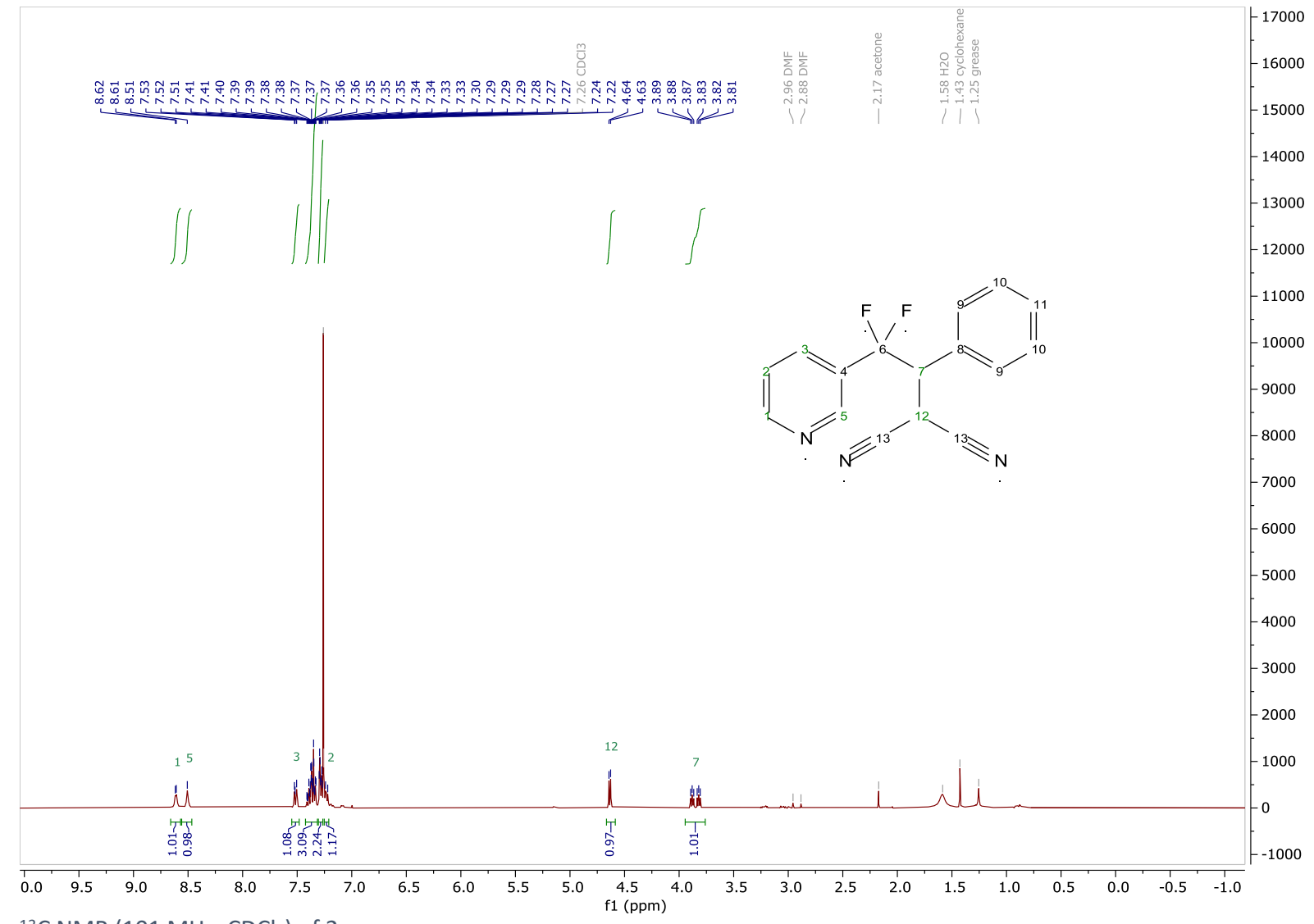

${ }^{13} \mathrm{C}$ NMR $\left(101 \mathrm{MHz}, \mathrm{CDCl}_{3}\right.$ ) of 2p:

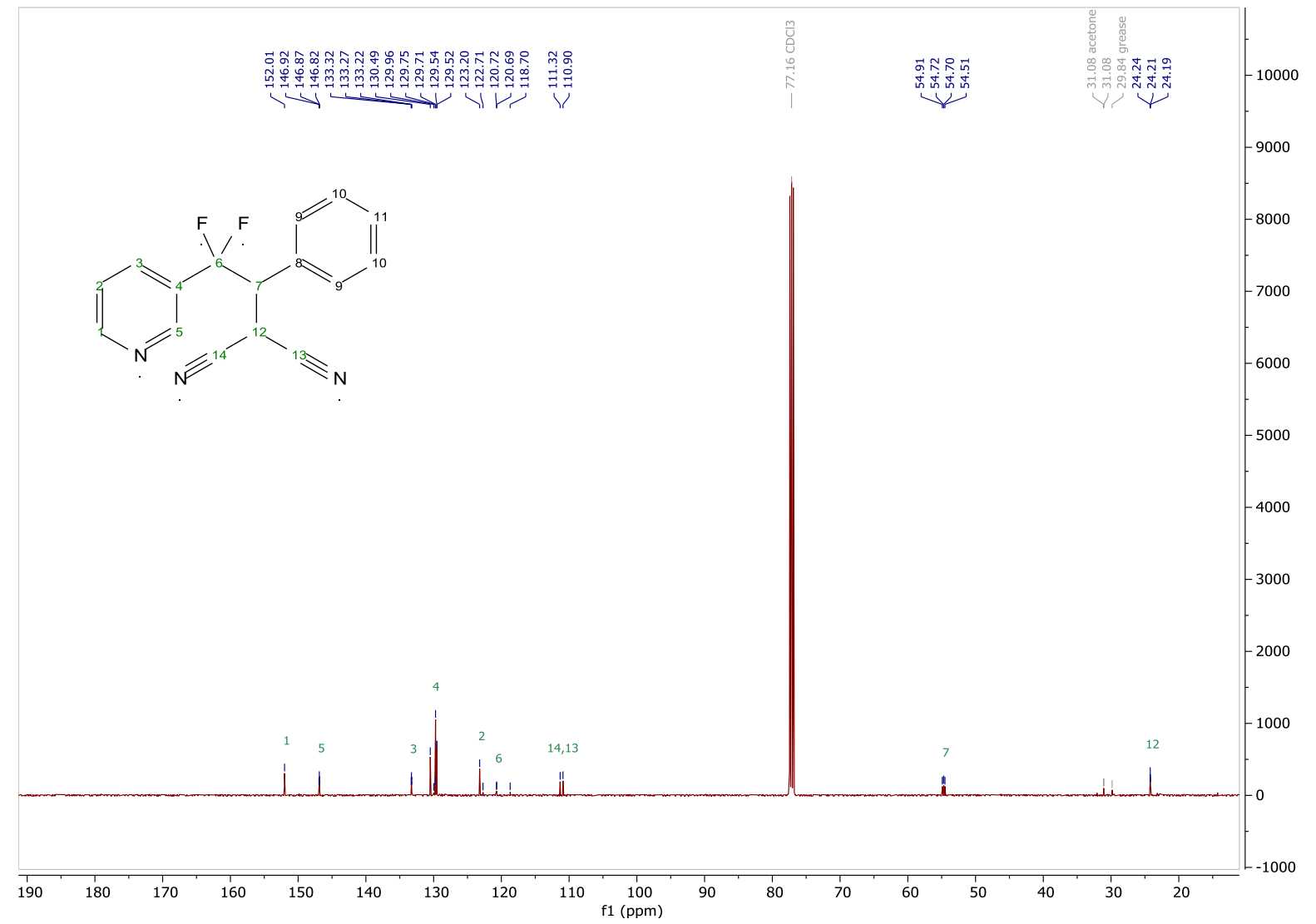




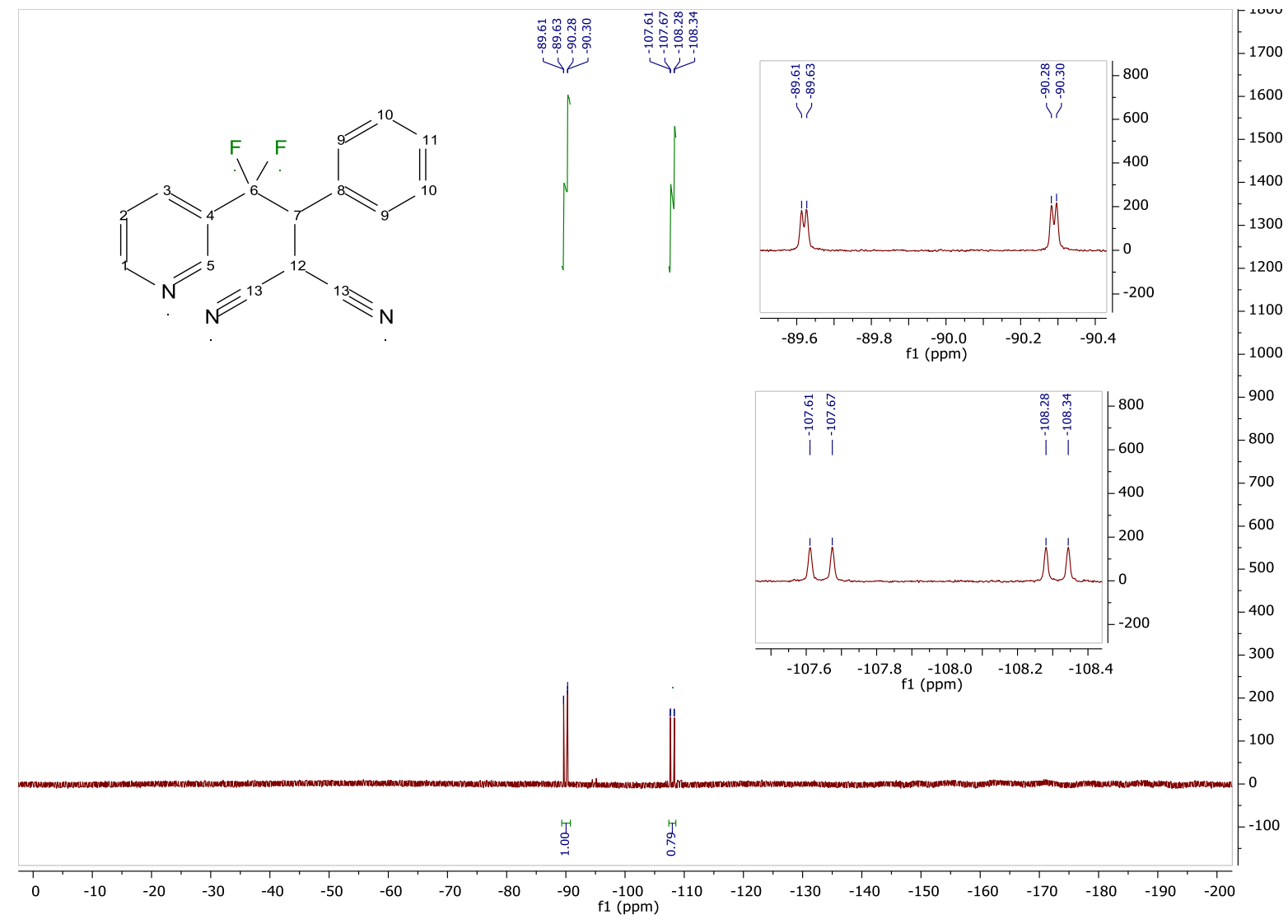

\section{3-(bromodifluoromethyl)pyridine (2q)}<smiles>FC(F)(Br)c1cccnc1</smiles>

\section{By sequential quench:}

2q was prepared according to protocol A using cyanogen bromide (1.5 equiv., $2.88 \mathrm{mmol}, 304 \mathrm{mg}$ ). $0 \%$ of ${ }^{19} \mathrm{~F}$ NMR yield was calculated with fluorobenzene as internal standard.

\section{By desilylative post-functionalization:}

Cyanogen bromide (1.5 equiv., $0.57 \mathrm{mmol}, 60 \mathrm{mg}$ ) was added to a solution of 3((dimethyl(phenyl)silyl)difluoromethyl)pyridine $\mathbf{2 b}$ (1 equiv., $0.35 \mathrm{mmol}, 92 \mathrm{mg}$ ) in DMF ( $1 \mathrm{~mL}$ ) at $0{ }^{\circ} \mathrm{C}$ and under inert atmosphere. A solution of TBAT (1 equiv., $0.37 \mathrm{mmol}, 200 \mathrm{mg}$ ) in DMF $(0.5 \mathrm{~mL}$ ) precooled to $0{ }^{\circ} \mathrm{C}$ was added to the reaction mixture, which was then stirred for $17 \mathrm{~h}$ at $70{ }^{\circ} \mathrm{C} .52 \%$ of ${ }^{19} \mathrm{~F}$ NMR yield was calculated with fluorobenzene as internal standard. Purification via flash column chromatography (silica gel, gradient from 0 to $15 \%$ of EtOAc in cyclohexane) yielded $2 q(29 \mathrm{mg}, 0.14$ $\mathrm{mmol}, 20 \%$ ). Purification from the starting material (2b) was not possible, traces of $2 \mathrm{~b}$ are present in ${ }^{1} \mathrm{H}$ NMR. Due to low stability under air atmosphere of $\mathbf{2 q}{ }^{13} \mathrm{C}$ NMR was not exploitable. 
${ }^{1} \mathrm{H}$ NMR (500 MHz, CDCl 3$): \delta 8.89(\mathrm{dd}, J=2.4,0.9 \mathrm{~Hz}, 1 \mathrm{H}, \mathrm{H5}), 8.74(\mathrm{dd}, J=4.9,1.6 \mathrm{~Hz}, 1 \mathrm{H}, \mathrm{H1}), 7.91$ (dtd, $J=$ 8.1, 1.6, $0.8 \mathrm{~Hz}, 1 \mathrm{H}, \mathrm{H} 3), 7.43-7.41(\mathrm{~m}, 1 \mathrm{H}, \mathrm{H} 2)$.

${ }^{19}$ F NMR (377 MHz, $\left.\mathrm{CDCl}_{3}\right): \delta-44.68(2 \mathrm{~F})$.

HRMS (ESI): $\mathrm{m} / \mathrm{z} ;[\mathrm{M}+\mathrm{H}]^{+}$calcd for $\mathrm{C}_{6} \mathrm{H}_{5} \mathrm{BrF}_{2} \mathrm{~N}$ : 207.9568; found: 207.9560 .

${ }^{1} \mathrm{H} \mathrm{NMR}\left(500 \mathrm{MHz}, \mathrm{CDCl}_{3}\right.$ ) of 2q:

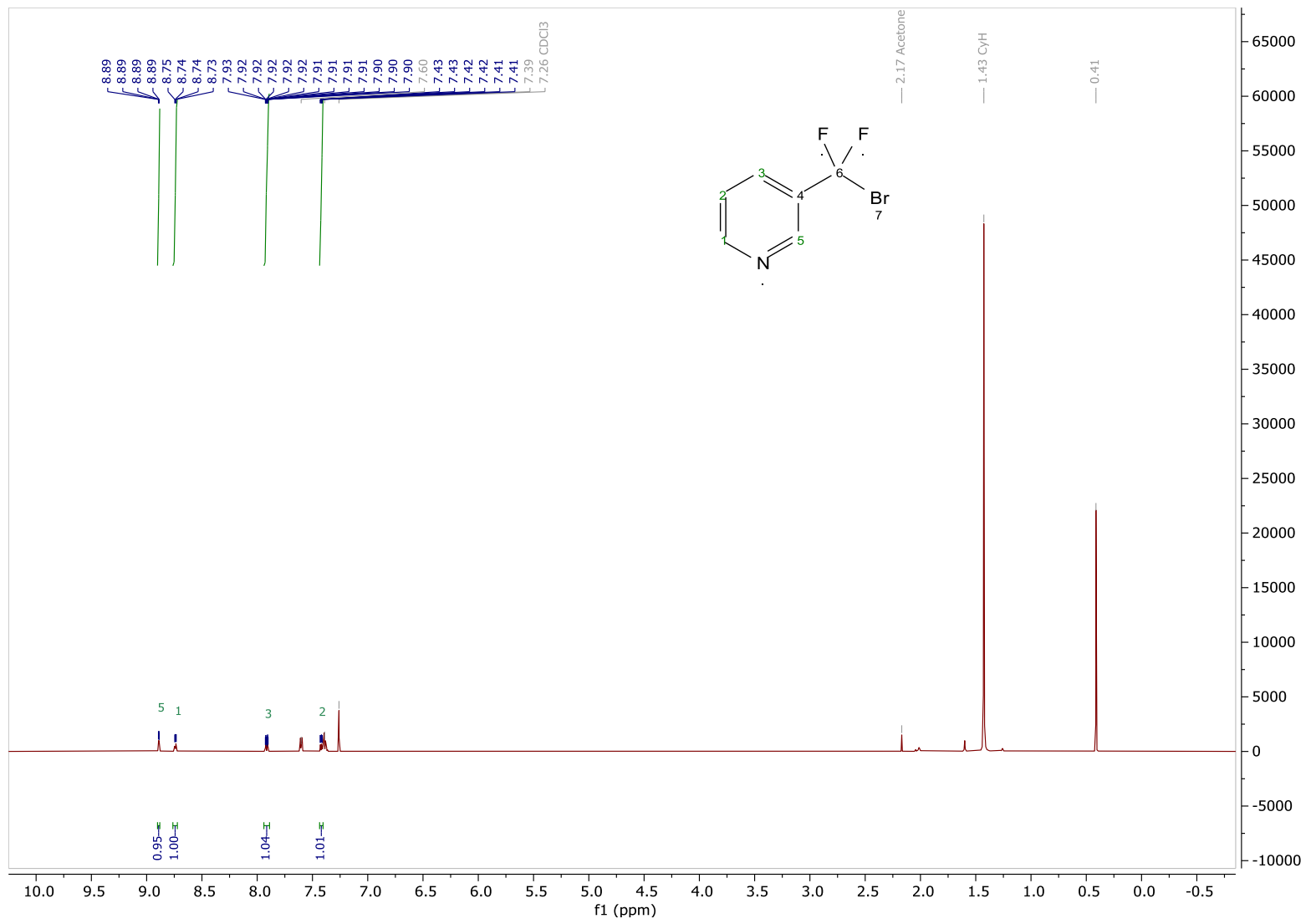




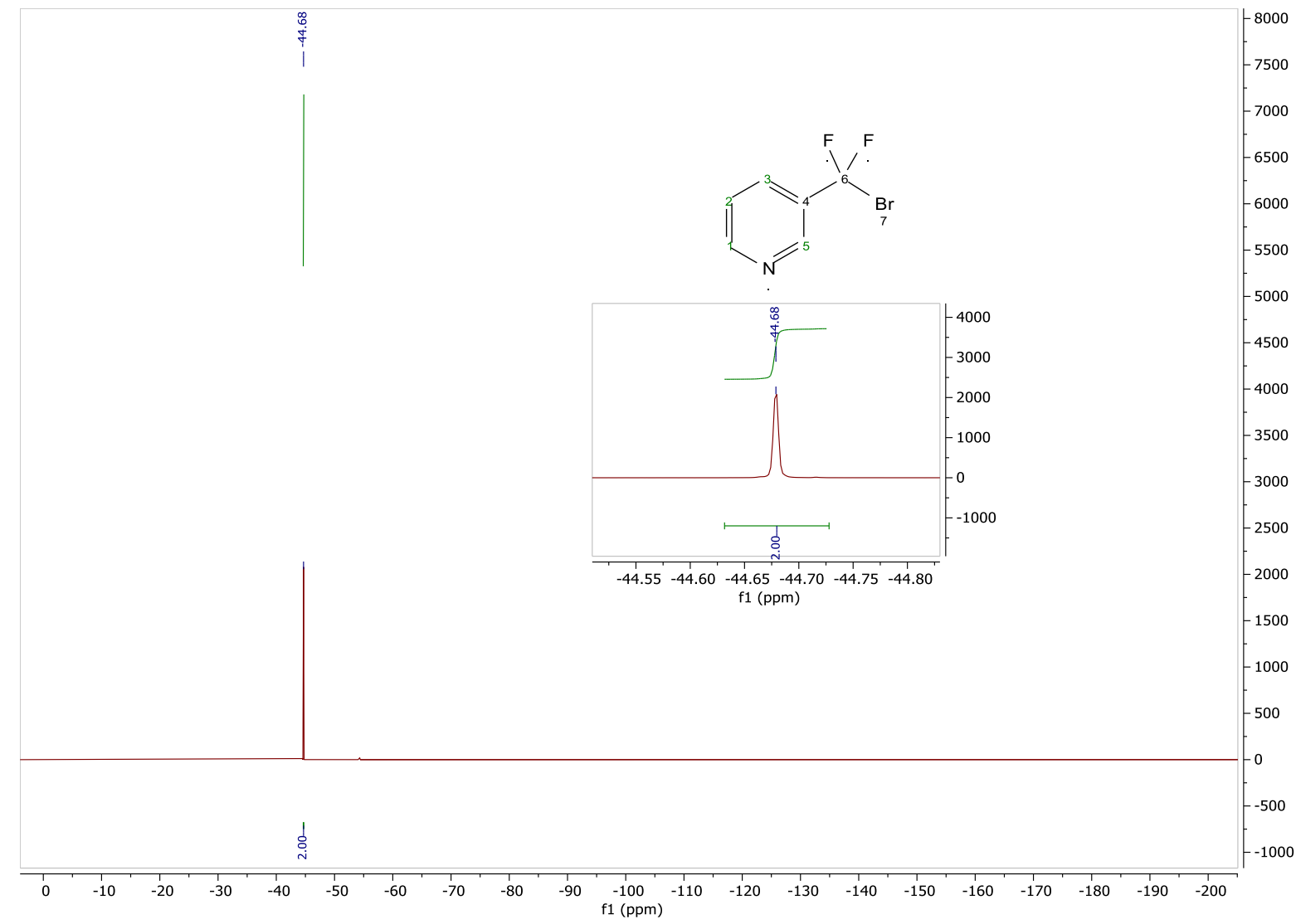

3-(difluoro(p-tolylsulfinyl)methyl)pyridine (2r)

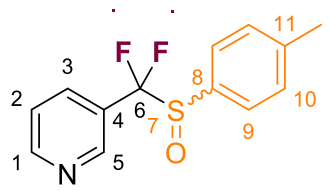

\section{By sequential quench:}

2r was prepared according to protocol A using (1R,2S,5R)-(-)-Menthyl ( $S)$-p-toluenesulfinate (1.5 equiv., $2.88 \mathrm{mmol}, 304 \mathrm{mg}$ ). $0 \%$ of ${ }^{19} \mathrm{~F} \mathrm{NMR}$ yield was calculated with fluorobenzene as internal standard.

\section{By desilylative post-functionalization:}

$(1 R, 2 S, 5 R)-(-)$-Menthyl (S)-p-toluenesulfinate (1.1 equiv., $0.48 \mathrm{mmol}, 140 \mathrm{mg})$ was added to a solution of 3-((dimethyl(phenyl)silyl)difluoromethyl)pyridine $\mathbf{2 b}$ (1 equiv., $0.41 \mathrm{mmol}, 108 \mathrm{mg}$ ) in DMF (1 mL) at $0{ }^{\circ} \mathrm{C}$ and under inert atmosphere. A solution of TBAT (1 equiv., $0.44 \mathrm{mmol}, 238 \mathrm{mg}$ ) in DMF $(0.5 \mathrm{~mL})$ pre-cooled to $0{ }^{\circ} \mathrm{C}$ was added to the reaction mixture, which was then stirred for $17 \mathrm{~h}$ at $70{ }^{\circ} \mathrm{C} .41 \%$ of ${ }^{19} \mathrm{~F}$ NMR yield was calculated with fluorobenzene as internal standard. Purification via flash column chromatography (silica gel, gradient from 0 to $25 \%$ of EtOAc in cyclohexane) yielded $2 r$ as a colourless oil (41 mg, $0.15 \mathrm{mmol}, 37 \%)$.

${ }^{1} \mathrm{H}$ NMR (400 MHz, $\left.\mathrm{CDCl}_{3}\right): \delta 8.69-8.62(\mathrm{~m}, 1 \mathrm{H}, \mathrm{H} 1), 8.11-8.04(\mathrm{~m}, 1 \mathrm{H}, \mathrm{H} 5), 7.51(\mathrm{dt}, J=8.0,1.8 \mathrm{~Hz}$, $1 \mathrm{H}, \mathrm{H} 3), 7.31-7.27(\mathrm{~m}, 1 \mathrm{H}, \mathrm{H} 2), 7.19(\mathrm{~d}, J=8.0 \mathrm{~Hz}, 2 \mathrm{H}, \mathrm{H} 9), 7.14$ (d, J = $8.3 \mathrm{~Hz}, 2 \mathrm{H}, \mathrm{H} 10) 2.37(\mathrm{~s}, 3 \mathrm{H}$, $\mathrm{H} 12)$. 
${ }^{13} \mathrm{C}$ NMR (101 MHz, CDCl 3$): \delta 152.4(\mathrm{C} 1), 147.4\left(\mathrm{t}, \mathrm{C}-\mathrm{F},{ }^{3} \mathrm{~J}_{\mathrm{C}-\mathrm{F}}=6.1 \mathrm{~Hz}, \mathrm{C} 5\right), 143.8$ (C11), $134.8\left(\mathrm{t}, \mathrm{C}-\mathrm{F},{ }^{3} \mathrm{~J}_{\mathrm{C}-\mathrm{F}}\right.$ $=5.6 \mathrm{~Hz}, \mathrm{C} 3), 133.8\left(\mathrm{t}, \mathrm{C}-\mathrm{F},{ }^{3} \mathrm{~J}_{\mathrm{C}-\mathrm{F}}=4.9 \mathrm{~Hz}, \mathrm{C} 8\right), 129.8$ (C10), $125.6(\mathrm{C} 9), 123.3\left(\mathrm{t}, \mathrm{C}-\mathrm{F},{ }^{2} J_{\mathrm{C}-\mathrm{F}}=22.7 \mathrm{~Hz}, \mathrm{C} 4\right)$, $122.6(\mathrm{C} 2), 113.4\left(\mathrm{t}, \mathrm{C}-\mathrm{F},{ }^{1} J_{\mathrm{C}-\mathrm{F}}=239.2 \mathrm{~Hz}, \mathrm{C} 6\right), 21.6(\mathrm{C} 12)$.

${ }^{19} \mathrm{~F}$ NMR (377 MHz, CDCl $\left.{ }_{3}\right): \delta-99.25\left(\mathrm{~d}, \mathrm{~F}-\mathrm{F},{ }^{2} \mathrm{~J}_{\mathrm{F}-\mathrm{F}}=236.0 \mathrm{~Hz}, 1 \mathrm{~F}\right),-104.74\left(\mathrm{~d}, \mathrm{~F}-\mathrm{F},{ }^{2} \mathrm{~J}_{\mathrm{F}-\mathrm{F}}=235.9 \mathrm{~Hz}, 1 \mathrm{~F}\right)$.

HRMS (ESI): $\mathrm{m} / \mathrm{z} ;[\mathrm{M}+\mathrm{H}]^{+}$calcd for $\mathrm{C}_{13} \mathrm{H}_{12} \mathrm{~F}_{2} \mathrm{NOS}$ : 268.0602; found: 268.0595.

${ }^{1} \mathrm{H}$ NMR $\left(400 \mathrm{MHz}, \mathrm{CDCl}_{3}\right)$ of $2 \mathrm{r}$ :

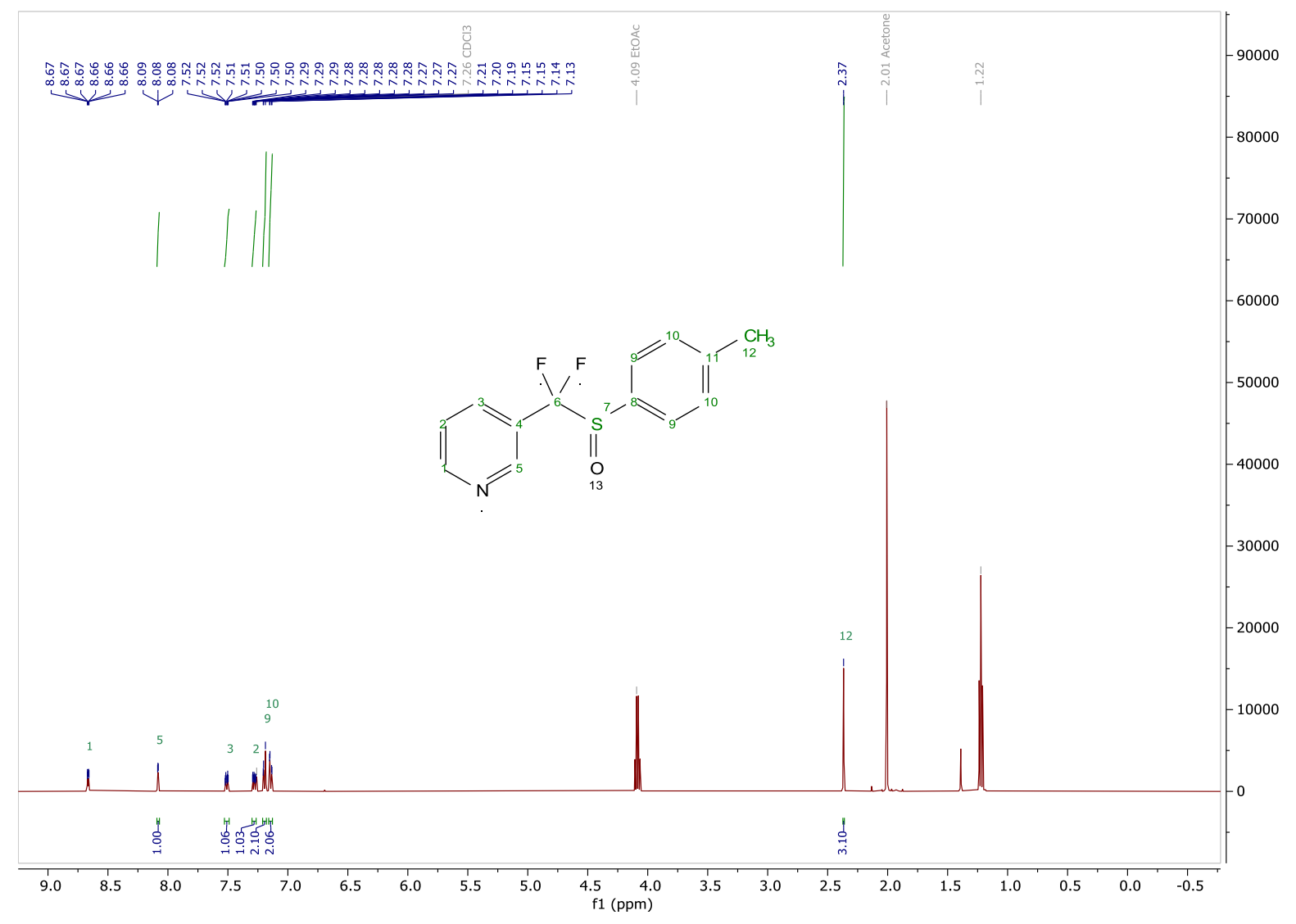


${ }^{13} \mathrm{C}$ NMR $\left(101 \mathrm{MHz}, \mathrm{CDCl}_{3}\right)$ of $2 \mathrm{r}$ :

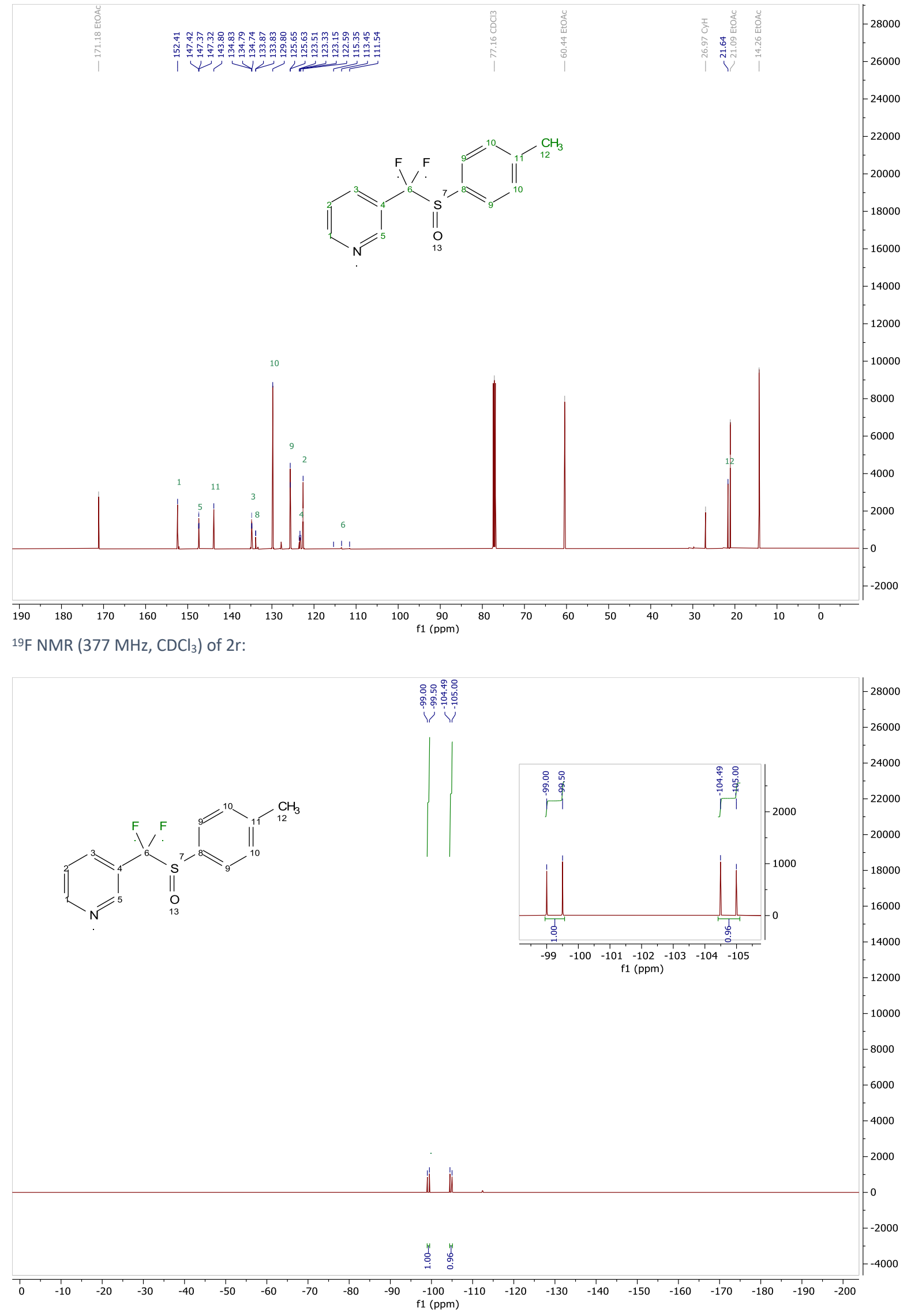

HPLC (ODH column, Hex/i-PrOH 98/2, $\left.0.5 \mathrm{~mL} / \mathrm{min}, 35^{\circ} \mathrm{C}\right): \mathrm{t}_{\mathrm{r}}=31.54 \mathrm{~min}(63 \%), \mathrm{t}_{\mathrm{r}}=38.83 \mathrm{~min}(37 \%)$. 
UAU

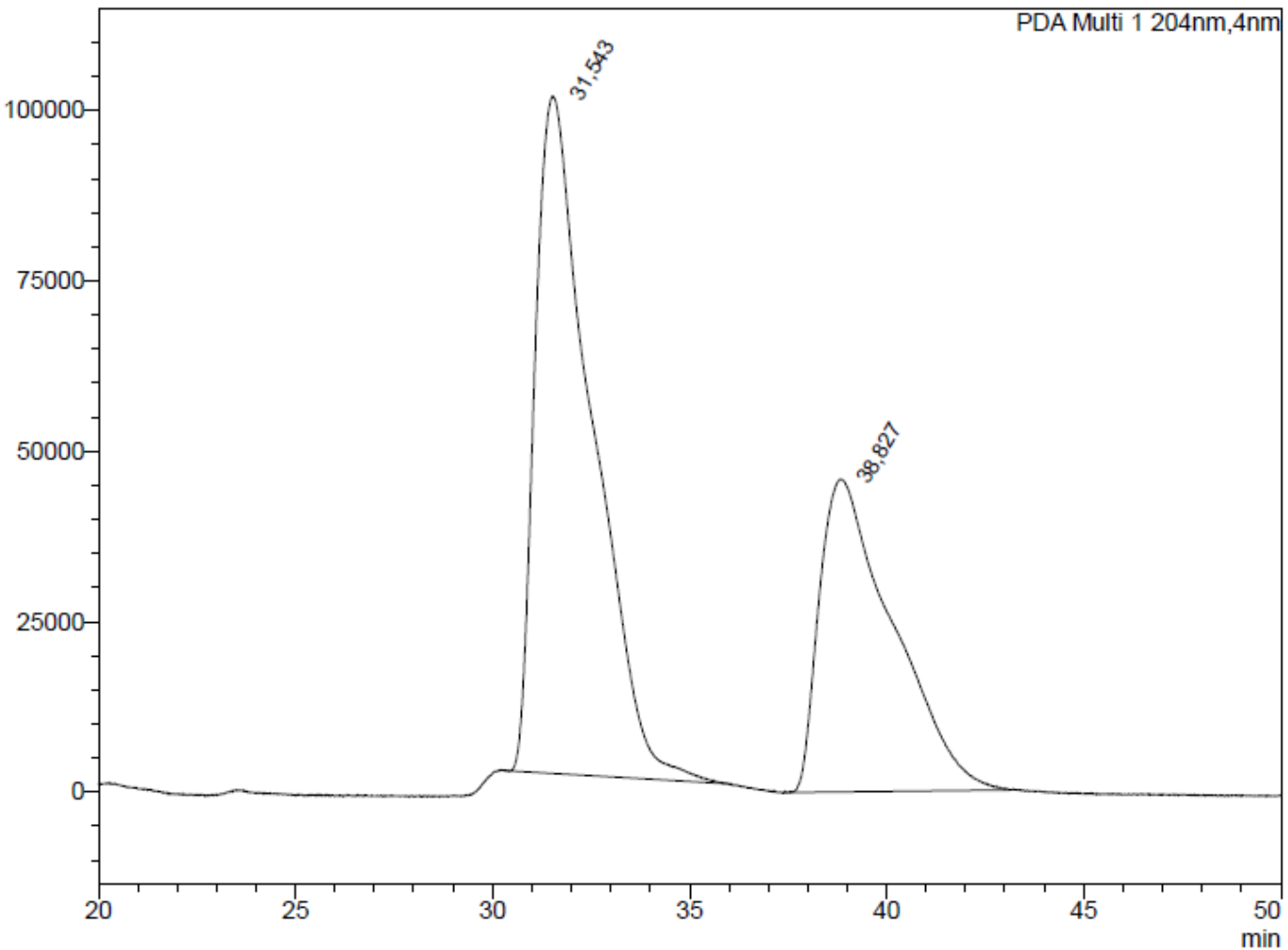

<PDA Chromatogram>

Peak Table

\begin{tabular}{|c|c|c|c|c|c|c|c|}
\hline Peak\# & Ret. Time & Area & Height & Area $\%$ & Capacity Factor $\left(k^{\prime}\right)$ & Resolution(USP) & Lambda max \\
\hline & $\frac{31,54}{3882}$ & $\frac{10007847}{554808}$ & 99338 & $\frac{62,695}{37305}$ & - & 100 & $203 / 221 / 261 / 488 / 403$ \\
\hline & & 15962655 & 145203 & $\frac{3,300}{100000}$ & & & \\
\hline
\end{tabular}

1-(2,4-dichlorophenyl)-2,2-difluoro-2-(pyridin-3-yl)ethan-1-one (3)<smiles>O=C(c1ccc(Cl)cc1Cl)C(F)(F)c1cccnc1</smiles>

To a solution of $2 r$ (1 equiv., $100 \mathrm{mg}, 0.33 \mathrm{mmol}$ ) in THF $(17 \mathrm{~mL})$, Dess-Martin periodinane (1.7 equiv., $242 \mathrm{mg}, 0.57 \mathrm{mmol}$ ) was added at $0{ }^{\circ} \mathrm{C}$. The reaction mixture was stirred at $0{ }^{\circ} \mathrm{C}$ for $50 \mathrm{~min}$ and then at $22{ }^{\circ} \mathrm{C}$ for $1 \mathrm{~h}$. The white slurry was quenched with a saturated solution of sodium thiosulfate. The reaction mixture was extracted with ethyl acetate, washed with sodium bicarbonate and then brine. The collected organic phase was dried over magnesium sulfate and filtrated. After removal of solvent, the residue was dried over vacuum. Purification via flash column chromatography (silica gel, gradient from 0 to $20 \%$ of EtOAc in cyclohexane) yielded 3 as a yellowish amorphous solid (62 $\mathrm{mg}, 0.21 \mathrm{mmol}$, $62 \%)$.

${ }^{1} \mathrm{H}$ NMR (400 MHz, $\left.\mathrm{CDCl}_{3}\right): \delta 8.80(\mathrm{dd}, J=2.3,1.0 \mathrm{~Hz}, 1 \mathrm{H}, \mathrm{H} 5), 8.79-8.75(\mathrm{~m}, 1 \mathrm{H}, \mathrm{H} 1), 7.88$ (dddd, $J=$ 7.4, 2.4, 1.6, $0.8 \mathrm{~Hz}, 1 \mathrm{H}, \mathrm{H} 2$ ), 7.50 (dt, J=8.4, 1.3 Hz, 1H, H12), 7.48 (d, J=1.9 Hz, 1H, H9), $7.45-7.40$ (m, $1 \mathrm{H}, \mathrm{H} 3), 7.35$ (dd, $J=8.3,2.0 \mathrm{~Hz}, 1 \mathrm{H}, \mathrm{H} 10)$. 
${ }^{13} \mathrm{C}$ NMR (101 MHz, CDCl $): \delta 190.2\left(\mathrm{t}, \mathrm{C}-\mathrm{F},{ }^{2} \mathrm{~J}_{\mathrm{C}-\mathrm{F}}=33.9 \mathrm{~Hz}, \mathrm{C7}\right), 152.4\left(\mathrm{t}, \mathrm{C}-\mathrm{F},{ }^{5} \mathrm{~J}_{\mathrm{C}-\mathrm{F}}=2.2 \mathrm{~Hz}, \mathrm{C} 1\right), 147.5$ (t, $\left.\mathrm{C}-\mathrm{F},{ }^{3} \mathrm{~J}_{\mathrm{C}-\mathrm{F}}=6.6 \mathrm{~Hz}, \mathrm{C} 5\right), 138.9$ (C11), 134.1 (t, C-F, $\left.{ }^{3} \mathrm{~J}_{\mathrm{C}-\mathrm{F}}=5.9 \mathrm{~Hz}, \mathrm{C} 3\right), 133.7$ (C13), 131.9 (C8), 130.9 (C12), 130.0 (C9), $128.0\left(\mathrm{t}, \mathrm{C}-\mathrm{F},{ }^{2} \mathrm{~J}_{\mathrm{C}-\mathrm{F}}=25.6 \mathrm{~Hz}, \mathrm{C} 4\right), 127.2$ (C10), 123.5 (C2), $115.4\left(\mathrm{t}, \mathrm{C}-\mathrm{F},{ }^{1} J_{\mathrm{C}-\mathrm{F}}=256.1 \mathrm{~Hz}, \mathrm{C} 6\right)$. ${ }^{19} \mathrm{~F}$ NMR (471 MHz, $\left.\mathrm{CDCl}_{3}\right): \delta-101.68(2 \mathrm{~F})$.

HRMS (ESI): $\mathrm{m} / \mathrm{z}[\mathrm{M}+\mathrm{H}]^{+}$calcd for $\mathrm{C}_{13} \mathrm{H}_{8} \mathrm{Cl}_{2} \mathrm{~F}_{2} \mathrm{NO}$ : 301.9946; found: 301.9944 .

${ }^{1} \mathrm{H}$ NMR (400 MHz, $\mathrm{CDCl}_{3}$ ) of $2 \mathrm{n}$ :

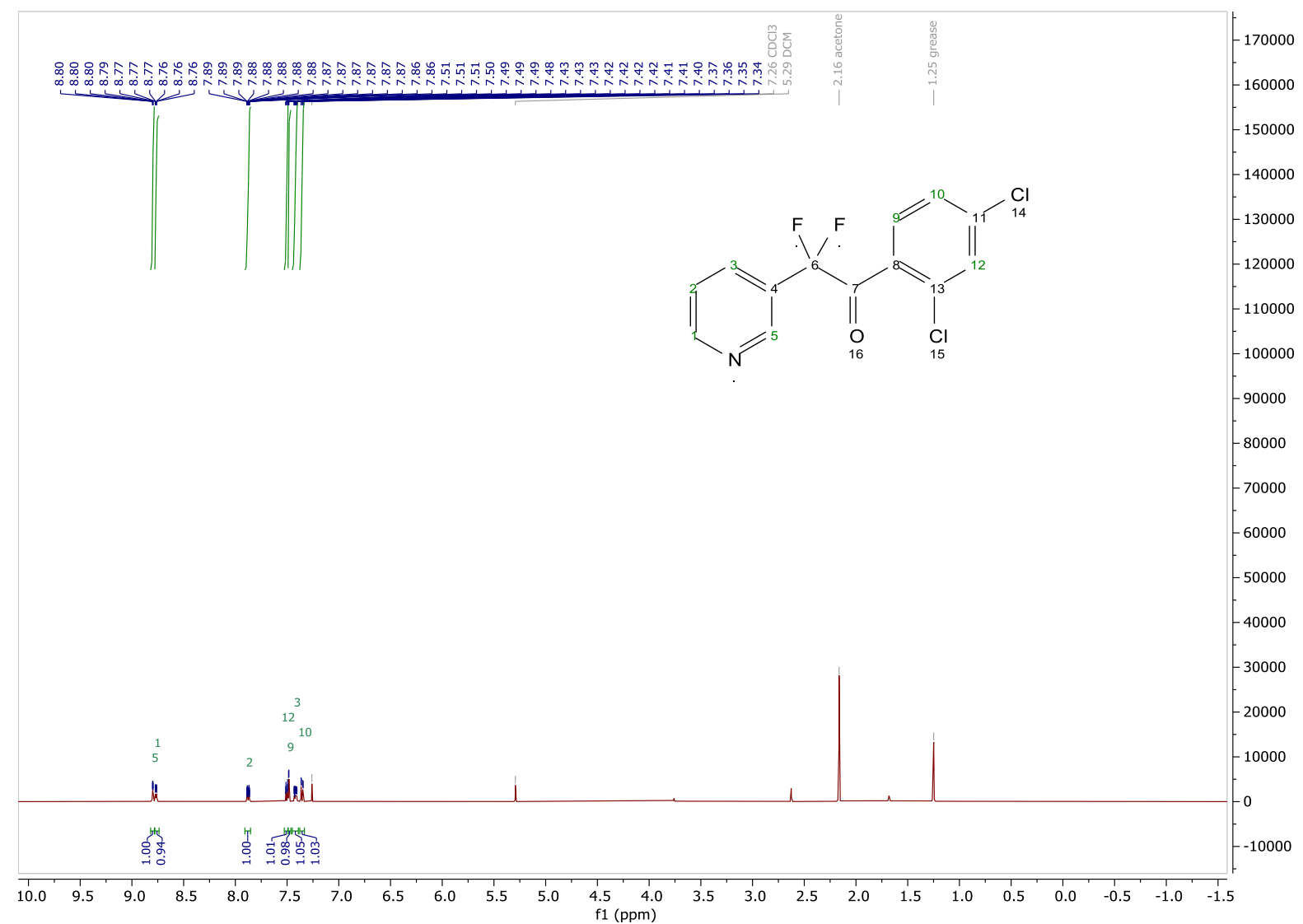


${ }^{13} \mathrm{C}$ NMR $\left(101 \mathrm{MHz}, \mathrm{CDCl}_{3}\right)$ of $2 \mathrm{n}$ :

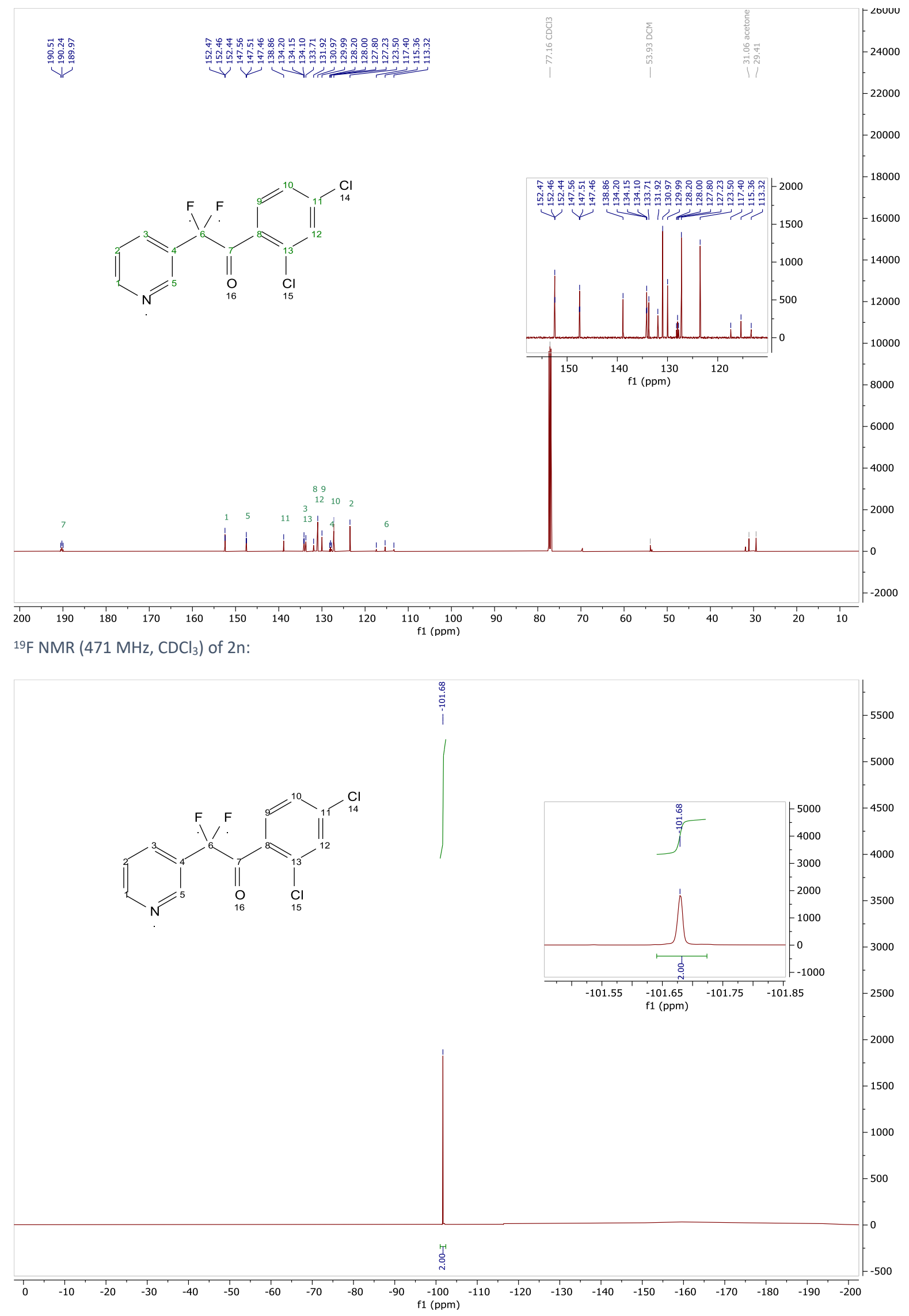


<smiles>CO/N=C(/c1ccc(Cl)cc1Cl)C(F)(F)c1cccnc1</smiles>

To a solution of 3 ( 1 equiv., $18 \mathrm{mg}, 0.06 \mathrm{mmol}$ ) in pyridine $(0.3 \mathrm{~mL}$ ) was added methoxyammonium chloride (4 equiv., $20 \mathrm{mg}, 0.24 \mathrm{mmol}$ ) in one portion at $20^{\circ} \mathrm{C}$. The colorless mixture was stirred at 50 ${ }^{\circ} \mathrm{C}$ for $16 \mathrm{~h}$. The pyridine was removed in vacuo. Water was added to the residue and the compound was extracted with ethyl acetate Organic phase was washed with brine, dried over magnesium sulfate and concentrated to afford a colorless oil. The mixture of geometrical oxime isomers 4 was pure enough ( $13 \mathrm{mg}, 0.04 \mathrm{mmol}, 66 \%$ ). A ratio of $75: 25$ of isomers was obtained.

\section{Major isomer}

${ }^{1} \mathrm{H}$ NMR (400 MHz, CDCl $): \delta 8.92-8.70(\mathrm{~m}, 2 \mathrm{H}, \mathrm{H} 1, \mathrm{H} 5), 7.87(\mathrm{~d}, J=7.9 \mathrm{~Hz}, 1 \mathrm{H}, \mathrm{H3}), 7.47(\mathrm{~d}, J=2.0 \mathrm{~Hz}$, $1 \mathrm{H}, \mathrm{H} 9), 7.42-7.39(\mathrm{~m}, 1 \mathrm{H}, \mathrm{H} 2), 7.34(\mathrm{dd}, J=8.3,2.1 \mathrm{~Hz}, 1 \mathrm{H}, \mathrm{H} 10), 7.21(\mathrm{~d}, J=8.2 \mathrm{~Hz}, 1 \mathrm{H}, \mathrm{H} 12), 3.83$ (s, 3H, H18).

${ }^{13} \mathrm{C}$ NMR (101 MHz, CDCl $): \delta 151.4$ (C1), 150.5 - 149.7 (m, C7), 147.9 (C5), 136.5 (C11), 134.4 (C8), $134.1\left(\mathrm{t}, \mathrm{C}-\mathrm{F},{ }^{3} \mathrm{~J}_{\mathrm{C}-\mathrm{F}}=5.7 \mathrm{~Hz}, \mathrm{C} 3\right), 131.0$ (C4), 130.5 (C10), 129.8 (C9), 127.3 (C12), 123.2 (C2), 117.6 (t, C$\left.\mathrm{F},{ }^{1} J_{\mathrm{C}-\mathrm{F}}=241.6 \mathrm{~Hz}, \mathrm{C} 6\right), 63.6(\mathrm{C} 18)$.

${ }^{19} \mathrm{~F}$ NMR (377 MHz, CDCl 3 ): $\delta-93.77\left(\mathrm{~d}, \mathrm{~F}-\mathrm{F},{ }^{2} \mathrm{~J}_{\mathrm{F}-\mathrm{F}}=279.4 \mathrm{~Hz}, 1 \mathrm{~F}\right),-95.34\left(\mathrm{~d}, \mathrm{~F}-\mathrm{F},{ }^{2} \mathrm{~J}_{\mathrm{F}-\mathrm{F}}=279.4 \mathrm{~Hz}, 1 \mathrm{~F}\right)$.

\section{Minor isomer}

${ }^{1}{ }_{H}$ NMR $\left(400 \mathrm{MHz}, \mathrm{CDCl}_{3}\right): \delta 8.92-8.70(\mathrm{~m}, 2 \mathrm{H}, \mathrm{H} 1, \mathrm{H} 5), 7.92(\mathrm{~d}, J=8.0 \mathrm{~Hz}, 1 \mathrm{H}, \mathrm{H3}), 7.51(\mathrm{~d}, J=2.0 \mathrm{~Hz}$, $1 \mathrm{H}, \mathrm{H} 9), 7.42-7.39(\mathrm{~m}, 1 \mathrm{H}, \mathrm{H} 2), 7.34$ (dd, $J=8.3,2.1 \mathrm{~Hz}, 1 \mathrm{H}, \mathrm{H} 10), 7.30$ (d, $J=8.2 \mathrm{~Hz}, 1 \mathrm{H}, \mathrm{H} 12), 3.88$ (s, 3H, H18).

${ }^{13} \mathrm{C}$ NMR (101 MHz, CDCl $): 151.4$ (C1), 150.5 - 149.6 (m, C7), 147.5 (C5), 136.7 (C11), 135.5 (C13), 134.4 (C8), 133.7 (C3), 132.5 (C10), 130.1 (C4), 127.3 (C9), 123.2 (C10), 117.6 (t, C-F, ${ }^{1} \mathrm{~J}_{\mathrm{C}-\mathrm{F}}=241.6 \mathrm{~Hz}$, C6), 63.6 (C18).

${ }^{19} \mathrm{~F}$ NMR (377 MHz, $\left.\mathrm{CDCl}_{3}\right): \delta-91.53(2 \mathrm{~F})$.

HRMS (ESI): $\mathrm{m} / \mathrm{z} ;[\mathrm{M}+\mathrm{H}]^{+}$calcd for $\mathrm{C}_{14} \mathrm{H}_{11} \mathrm{Cl}_{2} \mathrm{~F}_{2} \mathrm{~N}_{2} \mathrm{O}$ : 331.0211; found: 331.0218 . 
${ }^{1} \mathrm{H}$ NMR (400 MHz, $\mathrm{CDCl}_{3}$ ) of $2 \mathrm{n}$ :

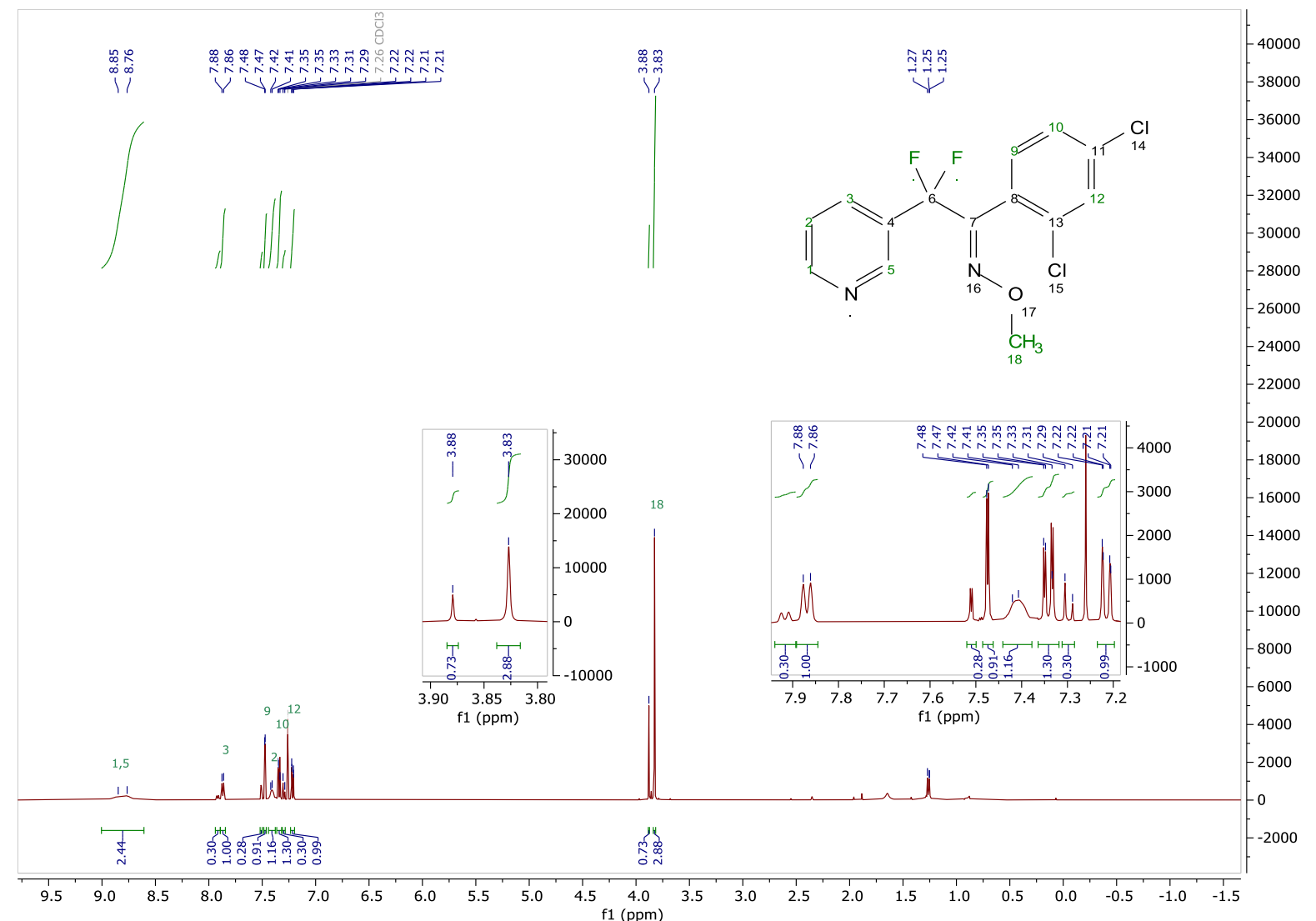

${ }^{13} \mathrm{C}$ NMR (101 MHz, $\mathrm{CDCl}_{3}$ ) of $2 \mathrm{n}$ :

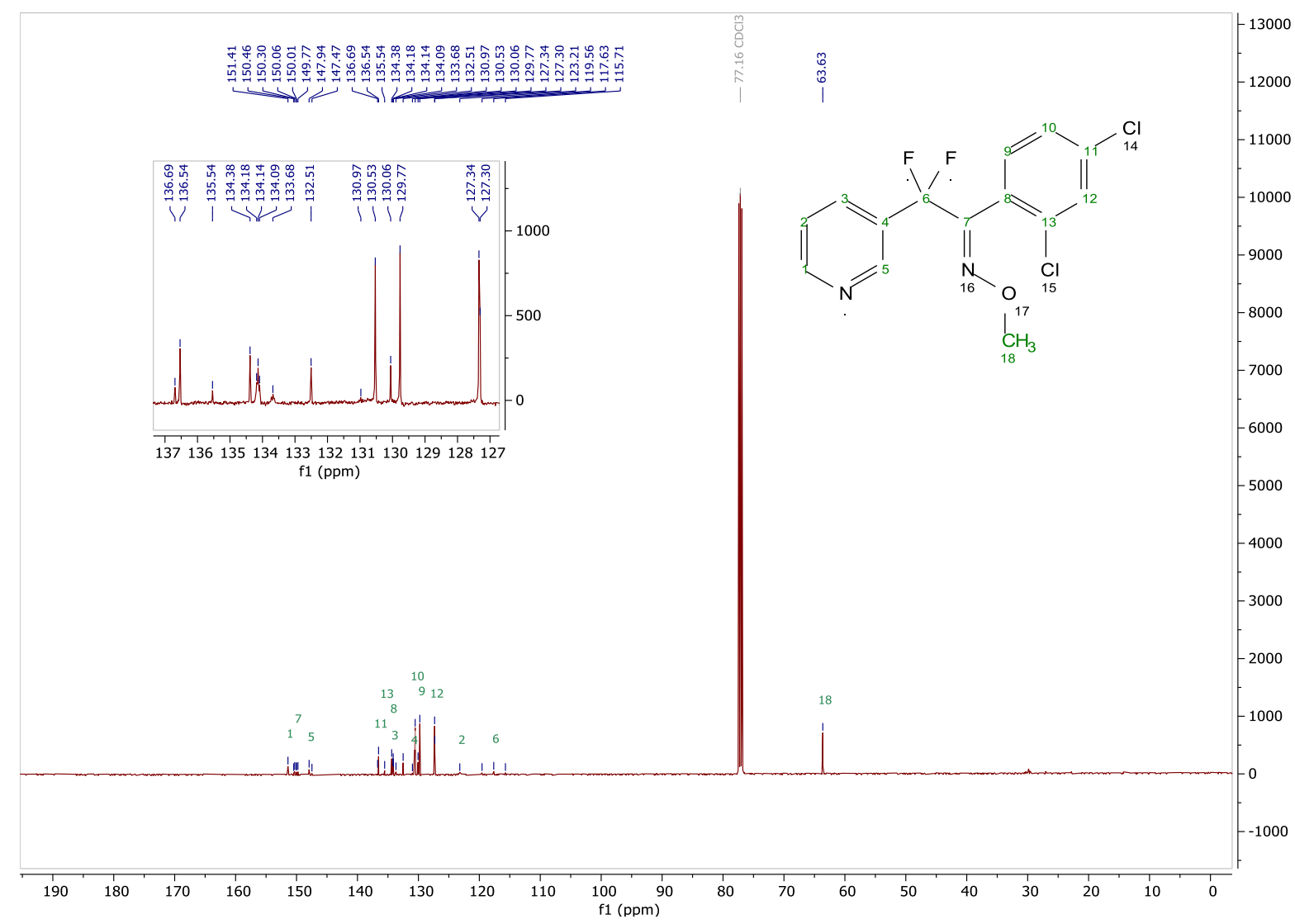




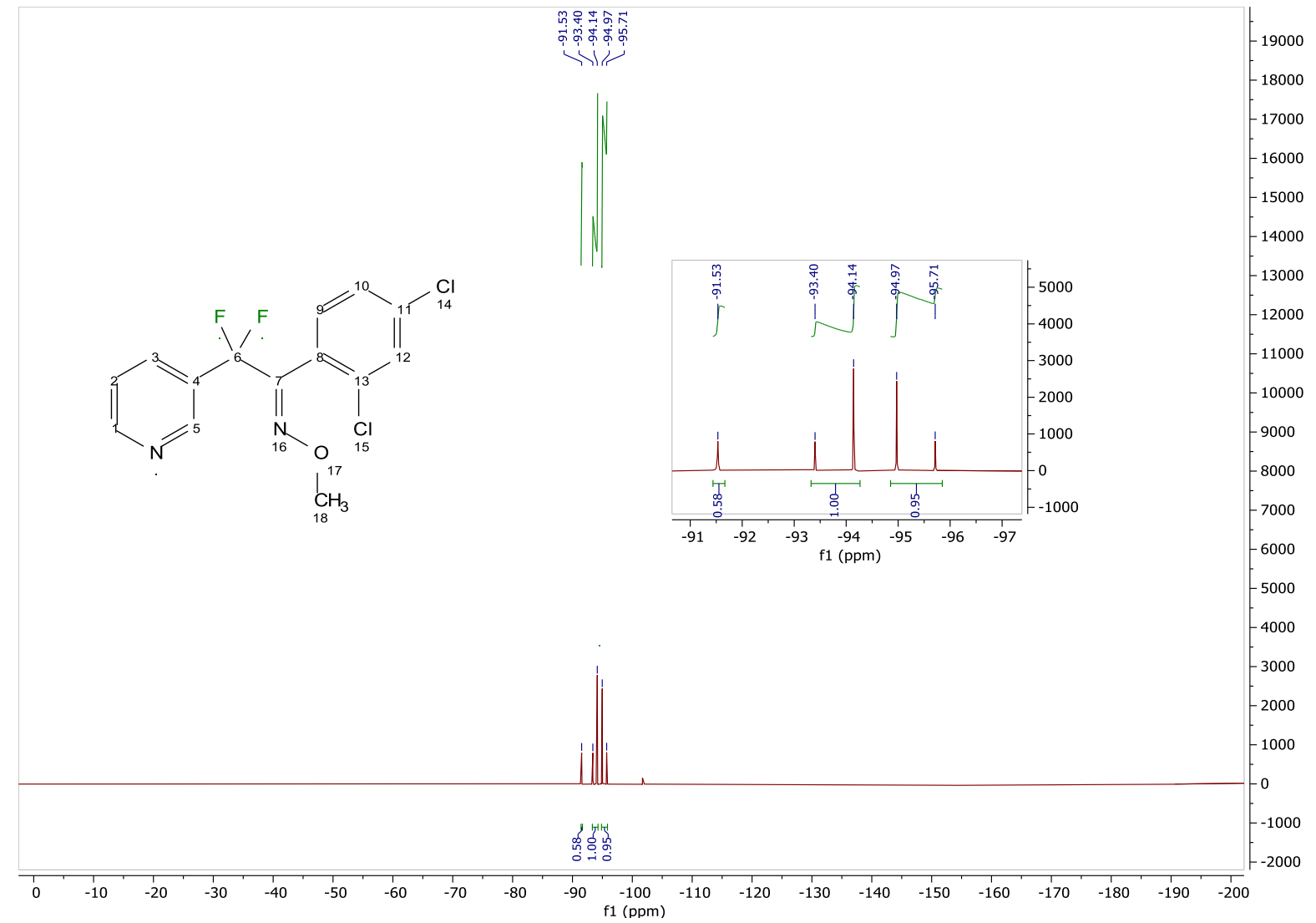

\section{X-Ray crystallographic structure of 21}

Crystalline $\mathbf{2 l}$ was obtained after slow concentration of sample in $\mathrm{CDCl}_{3}$.

X-Ray crystallographic experiments were performed by the Crystallography Service of the Université de Strasbourg.

The crystal-detector distance was $37 \mathrm{~mm}$. The cell parameters were determined (APEX3 software) ${ }^{7}$ from reflections taken from 1 set of 180 frames at $1 \mathrm{~s}$ exposure. The structure was solved using the program SHELXT-2014. ${ }^{8}$ The refinement and all further calculations were carried out using SHELXL$2014 .{ }^{8}$ The hydrogen atom of the $\mathrm{OH}$ group was located from Fourier difference. The other $\mathrm{H}$-atoms were included in calculated positions and treated as riding atoms using SHELXL default parameters. The non- $\mathrm{H}$ atoms were refined anisotropically, using weighted full-matrix least-squares on $\mathrm{F}^{2}$. A semiempirical absorption correction was applied using SADABS in APEX ${ }^{7}$; transmission factors: $T_{\min } / T_{\max }=$ $0.7025 / 0.7462$. 


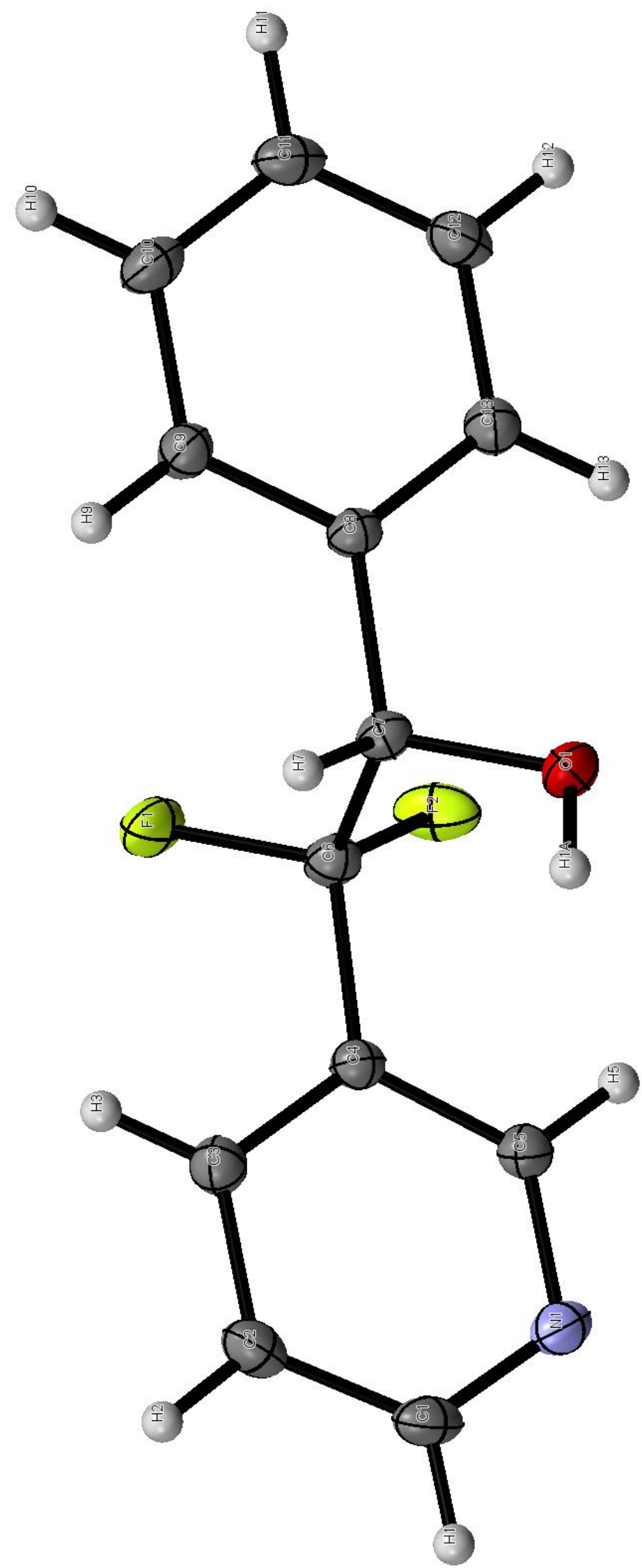

Image 1: thermal ellipsoid plot for crystalline 2 I with R1=3.6\% 
Table 1. Crystal data and structure refinement for $\mathbf{2 l}$

\begin{tabular}{|c|c|}
\hline Empirical formula & $\mathrm{C}_{13} \mathrm{H}_{11} \mathrm{~F}_{2} \mathrm{NO}$ \\
\hline Formula weight & 235.23 \\
\hline Temperature & $120(2) \mathrm{K}$ \\
\hline Wavelength & $0.71073 \mathrm{~A}$ \\
\hline Crystal system, space group & Monoclinic, $\mathrm{P} 21 / \mathrm{n}$ \\
\hline Unit cell dimensions & $\mathrm{a}=14.3672(4) \mathrm{A} \alpha=90 \mathrm{deg}$. \\
& $\mathrm{b}=5.46500(10) \mathrm{A} \quad \mathrm{\beta}=117.1820(10) \mathrm{deg}$. \\
$\mathrm{c}=15.4019(4) \mathrm{A} \quad \mathrm{V}=90 \mathrm{deg}$.
\end{tabular}

Table 2. Atomic coordinates $\left(\times 10^{4}\right)$ and equivalent isotropic displacement parameters $\left(A^{2} \times 10^{3}\right)$ for 2I. $U(e q)$ is defined as one third of the trace of the orthogonalized Uij tensor.

\begin{tabular}{|c|c|c|c|c|}
\hline & $x$ & $y$ & z & $U(e q)$ \\
\hline$C(1)$ & $1265(1)$ & $5092(2)$ & $5955(1)$ & $22(1)$ \\
\hline$C(2)$ & 1755(1) & $6896(2)$ & $5679(1)$ & $22(1)$ \\
\hline$C(3)$ & $2822(1)$ & $6679(2)$ & $5976(1)$ & 19(1) \\
\hline$C(4)$ & $3349(1)$ & $4658(2)$ & 6534(1) & $15(1)$ \\
\hline$C(5)$ & 2783(1) & $2896(2)$ & $6745(1)$ & $18(1)$ \\
\hline$C(6)$ & $4521(1)$ & $4458(2)$ & $6958(1)$ & $17(1)$ \\
\hline$C(7)$ & $5110(1)$ & $5798(2)$ & 7944(1) & $16(1)$ \\
\hline$C(8)$ & $6284(1)$ & $5602(2)$ & $8342(1)$ & $15(1)$ \\
\hline$C(9)$ & $6818(1)$ & $7431(2)$ & $8122(1)$ & 18(1) \\
\hline$C(10)$ & 7897(1) & $7287(2)$ & $8462(1)$ & $22(1)$ \\
\hline$C(11)$ & $8447(1)$ & $5302(2)$ & $9028(1)$ & $23(1)$ \\
\hline$C(12)$ & $7921(1)$ & $3477(2)$ & $9258(1)$ & $22(1)$ \\
\hline$C(13)$ & $6841(1)$ & $3621(2)$ & $8917(1)$ & 19(1) \\
\hline$N(1)$ & 1753(1) & $3090(2)$ & 6459(1) & $21(1)$ \\
\hline
\end{tabular}


$\mathrm{O}(1) \quad 4787(1) \quad 4820(1) \quad 8612(1) \quad 20(1)$

$F(1) \quad 4838(1) \quad 5407(1) \quad 6312(1) \quad 25(1)$

$F(2) \quad 4807(1) \quad 2037(1) \quad 7067(1) \quad 25(1)$

Table 3. Bond lengths [A] and angles [deg] for $\mathbf{2 1}$.

$\begin{array}{ll}\mathrm{C}(1)-\mathrm{N}(1) & 1.3393(13) \\ \mathrm{C}(1)-\mathrm{C}(2) & 1.3859(13) \\ \mathrm{C}(1)-\mathrm{H}(1) & 0.9500 \\ \mathrm{C}(2)-\mathrm{C}(3) & 1.3905(11) \\ \mathrm{C}(2)-\mathrm{H}(2) & 0.9500 \\ \mathrm{C}(3)-\mathrm{C}(4) & 1.3921(11) \\ \mathrm{C}(3)-\mathrm{H}(3) & 0.9500 \\ \mathrm{C}(4)-\mathrm{C}(5) & 1.3917(11) \\ \mathrm{C}(4)-\mathrm{C}(6) & 1.5057(11) \\ \mathrm{C}(5)-\mathrm{N}(1) & 1.3416(10) \\ \mathrm{C}(5)-\mathrm{H}(5) & 0.9500 \\ \mathrm{C}(6)-\mathrm{F}(1) & 1.3709(9) \\ \mathrm{C}(6)-\mathrm{F}(2) & 1.3726(10) \\ \mathrm{C}(6)-\mathrm{C}(7) & 1.5446(12) \\ \mathrm{C}(7)-\mathrm{O}(1) & 1.4120(9) \\ \mathrm{C}(7)-\mathrm{C}(8) & 1.5127(10) \\ \mathrm{C}(7)-H(7) & 1.0000 \\ \mathrm{C}(8)-\mathrm{C}(9) & 1.3921(11) \\ \mathrm{C}(8)-\mathrm{C}(13) & 1.3965(11) \\ \mathrm{C}(9)-\mathrm{C}(10) & 1.3938(11) \\ \mathrm{C}(9)-H(9) & 0.9500 \\ \mathrm{C}(10)-\mathrm{C}(11) & 1.3897(14) \\ \mathrm{C}(10)-\mathrm{H}(10) & 0.9500 \\ \mathrm{C}(11)-\mathrm{C}(12) & 1.3918(13) \\ \mathrm{C}(11)-\mathrm{H}(11) & 0.9500 \\ \mathrm{C}(12)-\mathrm{C}(13) & 1.3947(11) \\ \mathrm{C}(12)-\mathrm{H}(12) & 0.9500 \\ \mathrm{C}(13)-\mathrm{H}(13) & 0.9500 \\ \mathrm{O}(1)-\mathrm{H}(1 \mathrm{~A}) & 0.943(18) \\ & \\ \mathrm{N}(1)-\mathrm{C}(1)-\mathrm{C}(2) & 123.67(8) \\ \mathrm{N}(1)-\mathrm{C}(1)-\mathrm{H}(1) & 118.2 \\ \mathrm{C}(2)-\mathrm{C}(1)-\mathrm{H}(1) & 118.2 \\ \mathrm{C}(1)-\mathrm{C}(2)-\mathrm{C}(3) & 118.45(8) \\ \mathrm{C}(1)-\mathrm{C}(2)-\mathrm{H}(2) & 120.8 \\ \mathrm{C}(3)-\mathrm{C}(2)-\mathrm{H}(2) & 120.8 \\ \mathrm{C}(2)-\mathrm{C}(3)-\mathrm{C}(4) & 118.57(8) \\ \mathrm{C}(2)-\mathrm{C}(3)-\mathrm{H}(3) & 120.7 \\ \mathrm{C}(4)-\mathrm{C}(3)-\mathrm{H}(3) & 120.7 \\ \mathrm{C}(5)-\mathrm{C}(4)-\mathrm{C}(3) & 118.79(7) \\ \mathrm{C}(5)-\mathrm{C}(4)-\mathrm{C}(6) & 120.37(7) \\ \mathrm{C}(3)-\mathrm{C}(4)-\mathrm{C}(6) & 120.72(7) \\ \mathrm{N}(1)-\mathrm{C}(5)-\mathrm{C}(4) & 122.94(8) \\ & \end{array}$




$\begin{array}{ll}N(1)-C(5)-H(5) & 118.5 \\ C(4)-C(5)-H(5) & 118.5 \\ F(1)-C(6)-F(2) & 105.91(6) \\ F(1)-C(6)-C(4) & 109.53(6) \\ F(2)-C(6)-C(4) & 109.61(7) \\ F(1)-C(6)-C(7) & 108.62(7) \\ F(2)-C(6)-C(7) & 109.72(7) \\ C(4)-C(6)-C(7) & 113.18(6) \\ O(1)-C(7)-C(8) & 110.78(6) \\ O(1)-C(7)-C(6) & 108.79(7) \\ C(8)-C(7)-C(6) & 111.95(6) \\ O(1)-C(7)-H(7) & 108.4 \\ C(8)-C(7)-H(7) & 108.4 \\ C(6)-C(7)-H(7) & 108.4 \\ C(9)-C(8)-C(13) & 119.46(7) \\ C(9)-C(8)-C(7) & 119.11(7) \\ C(13)-C(8)-C(7) & 121.42(7) \\ C(8)-C(9)-C(10) & 120.61(8) \\ C(8)-C(9)-H(9) & 119.7 \\ C(10)-C(9)-H(9) & 119.7 \\ C(11)-C(10)-C(9) & 119.77(8) \\ C(11)-C(10)-H(10) & 120.1 \\ C(9)-C(10)-H(10) & 120.1 \\ C(10)-C(11)-C(12) & 119.96(8) \\ C(10)-C(11)-H(11) & 120.0 \\ C(12)-C(11)-H(11) & 120.0 \\ C(11)-C(12)-C(13) & 120.27(8) \\ C(11)-C(12)-H(12) & 119.9 \\ C(13)-C(12)-H(12) & 119.9 \\ C(12)-C(13)-C(8) & 119.92(8) \\ C(12)-C(13)-H(13) & 120.0 \\ C(8)-C(13)-H(13) & 120.0 \\ C(1)-N(1)-C(5) & 117.49(8) \\ C(7)-O(1)-H(1 A) & 107.2(10) \\ & \\ & \end{array}$

Symmetry transformations used to generate equivalent atoms:

Table 4. Anisotropic displacement parameters $\left(\mathrm{A}^{2} \times 10^{3}\right)$ for $\mathbf{2}$. The anisotropic displacement factor exponent takes the form:

$$
-2 \mathrm{pi}^{2}\left[h^{2} a^{* \wedge} 2 \mathrm{U} 11+\ldots+2 h k a * b * U 12\right]
$$

\begin{tabular}{|c|c|c|c|c|c|c|}
\hline & U11 & $\mathrm{U} 22$ & U33 & U23 & U13 & U12 \\
\hline C (1) & $13(1)$ & $34(1)$ & 15 (1) & $-3(1)$ & $5(1)$ & $0(1)$ \\
\hline$C(2)$ & $19(1)$ & $27(1)$ & $17(1)$ & 2 (1) & $6(1)$ & $7(1)$ \\
\hline$C(3)$ & $19(1)$ & $20(1)$ & $19(1)$ & $2(1)$ & $10(1)$ & $1(1)$ \\
\hline$C(4)$ & $13(1)$ & $18(1)$ & $15(1)$ & $-1(1)$ & $6(1)$ & $0(1)$ \\
\hline$C(5)$ & $16(1)$ & 21 (1) & $16(1)$ & 1 (1) & $7(1)$ & $-1(1)$ \\
\hline$C(6)$ & $14(1)$ & $19(1)$ & $18(1)$ & $0(1)$ & $9(1)$ & 1 (1) \\
\hline$C(7)$ & $14(1)$ & $17(1)$ & $17(1)$ & $0(1)$ & $8(1)$ & $1(1)$ \\
\hline
\end{tabular}




\begin{tabular}{llllrrr}
$C(8)$ & $13(1)$ & $17(1)$ & $16(1)$ & $-1(1)$ & $7(1)$ & $0(1)$ \\
$C(9)$ & $17(1)$ & $19(1)$ & $21(1)$ & $1(1)$ & $10(1)$ & $-1(1)$ \\
$C(10)$ & $17(1)$ & $27(1)$ & $25(1)$ & $-2(1)$ & $12(1)$ & $-5(1)$ \\
$C(11)$ & $13(1)$ & $34(1)$ & $19(1)$ & $-4(1)$ & $6(1)$ & $0(1)$ \\
$C(12)$ & $18(1)$ & $28(1)$ & $18(1)$ & $2(1)$ & $6(1)$ & $6(1)$ \\
$C(13)$ & $18(1)$ & $19(1)$ & $19(1)$ & $2(1)$ & $9(1)$ & $1(1)$ \\
N $(1)$ & $16(1)$ & $30(1)$ & $18(1)$ & $-2(1)$ & $8(1)$ & $-5(1)$ \\
O $(1)$ & $17(1)$ & $26(1)$ & $21(1)$ & $3(1)$ & $12(1)$ & $2(1)$ \\
$F(1)$ & $18(1)$ & $42(1)$ & $20(1)$ & $0(1)$ & $12(1)$ & $-2(1)$ \\
$F(2)$ & $18(1)$ & $20(1)$ & $33(1)$ & $-6(1)$ & $7(1)$ & $4(1)$ \\
\hline
\end{tabular}

Table 5. Hydrogen coordinates $\left(\times 10^{4}\right)$ and isotropic displacement parameters $\left(\mathrm{A}^{2} \times 10^{3}\right)$ for $\mathbf{2 l}$.

\begin{tabular}{lllll}
\hline & $x$ & $y$ & $z$ & $U($ eq $)$ \\
& & & & \\
\hline H (1) & 543 & 5286 & 5777 & 26 \\
H (2) & 1370 & 8250 & 5296 & 26 \\
H (3) & 3183 & 7884 & 5801 & 23 \\
H (5) & 3142 & 1492 & 7107 & 22 \\
H (7) & 4913 & 7568 & 7842 & 19 \\
H (9) & 6443 & 8790 & 7736 & 22 \\
H (10) & 8255 & 8541 & 8308 & 27 \\
H (11) & 9182 & 5190 & 9256 & 27 \\
H (12) & 8299 & 2128 & 9649 & 26 \\
H (13) & 6485 & 2372 & 9076 & 22 \\
H (1A) & $4196(14)$ & $5720(30)$ & $8539(12)$ & $50(5)$ \\
& & & & \\
\hline
\end{tabular}

Table 6. Torsion angles [deg] for $\mathbf{2 l}$.

$N(1)-C(1)-C(2)-C(3)$
$C(1)-C(2)-C(3)-C(4)$
$C(2)-C(3)-C(4)-C(5)$
$C(2)-C(3)-C(4)-C(6)$
$C(3)-C(4)-C(5)-N(1)$
$C(6)-C(4)-C(5)-N(1)$
$C(5)-C(4)-C(6)-F(1)$
$C(3)-C(4)-C(6)-F(1)$
$C(5)-C(4)-C(6)-F(2)$
$C(3)-C(4)-C(6)-F(2)$
$C(5)-C(4)-C(6)-C(7)$
$C(3)-C(4)-C(6)-C(7)$
$F(1)-C(6)-C(7)-O(1)$
$F(2)-C(6)-C(7)-O(1)$
$C(4)-C(6)-C(7)-O(1)$
$F(1)-C(6)-C(7)-C(8)$
$F(2)-C(6)-C(7)-C(8)$
$C(4)-C(6)-C(7)-C(8)$
$O(1)-C(7)-C(8)-C(9)$
$C(6)-C(7)-C(8)-C(9)$
$O(1)-C(7)-C(8)-C(13)$

$$
\begin{array}{r}
-2.87(13) \\
0.14(12) \\
2.17(12) \\
-173.90(7) \\
-2.10(12) \\
173.99(7) \\
147.49(8) \\
-36.50(10) \\
31.69(10) \\
-152.29(7) \\
-91.14(9) \\
84.87(9) \\
-179.85(6) \\
-64.51(8) \\
58.27(9) \\
-57.09(9) \\
58.25(9) \\
-178.97(6) \\
-145.94(7) \\
92.43(9) \\
34.58(10)
\end{array}
$$




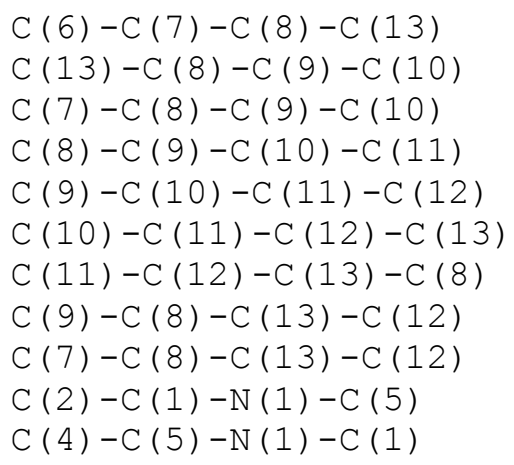

Symmetry transformations used to generate equivalent atoms: 
Table 7. Hydrogen bonds for 2 l [A and deg.].

\begin{tabular}{|c|c|c|c|c|}
\hline $\mathrm{D}-\mathrm{H} \ldots \mathrm{A}$ & $\mathrm{d}(\mathrm{D}-\mathrm{H})$ & $\mathrm{d}(\mathrm{H} \ldots \mathrm{A})$ & $\mathrm{d}(\mathrm{D} \ldots \mathrm{A})$ & $<(\mathrm{DHA})$ \\
\hline $\mathrm{O}(1)-\mathrm{H}(1 \mathrm{~A}) \ldots \mathrm{N}(1) \# 1$ & $0.943(18)$ & $1.882(18)$ & $2.8077(10)$ & $166.6(16)$ \\
\hline
\end{tabular}

Symmetry transformations used to generate equivalent atoms:

$\# 1-x+1 / 2, y+1 / 2,-z+3 / 2$ 


\section{References}

(1) Schlosser, M. Organometallics in Synthesis, second ed.; M. Schlosser, 2002.

(2) Aycock, D. F. Solvent Applications of 2-Methyltetrahydrofuran in Organometallic and Biphasic Reactions. Org. Process Res. Dev. 2007, 11, 156-159.

(3) Leal Silva, J. F.; Mariano, A. P.; Maciel Filho, R. Economic Potential of 2-Methyltetrahydrofuran (MTHF) and Ethyl Levulinate (EL) Produced from Hemicelluloses-Derived Furfural. Biomass Bioenergy 2018, 119, 492-502.

(4) Haas, A.; Spitzer, M.; Lieb, M. Synthese seitenkettenfluorierter aromatischer Verbindungen und deren chemische Reaktivität. Chem. Ber. 1988, 121, 1329-1340.

(5) Denisenko, I.; Oransky, D. A.; Varzatskii, O. A. First Example of Palladium Catalysis of CopperMediated Reactions Hetarylhalides with Ethyl Bromodifluoroacetate. Ukr. Khimicheskii Zhurnal Russ. Ed. 2015, 81, 19-24.

(6) Geri, J. B.; Wade Wolfe, M. M.; Szymczak, N. K. The Difluoromethyl Group as a Masked Nucleophile: A Lewis Acid/Base Approach. J. Am. Chem. Soc. 2018, 140, 9404-9408.

(7) M86-EXX229V1 APEX3 User Manual, Bruker AXS Inc., Madison, USA; 2016.

(8) Sheldrick, G. M. SHELXT - Integrated Space-Group and Crystal-Structure Determination. Acta Crystallogr. Sect. Found. Adv. 2015, 71, 3-8. 